\title{
Note on solutions of scattering equations
}

\author{
Bo Feng $\odot,{ }^{1,2,3,4, *}$ Chang $\mathrm{Hu},{ }^{1,5, \dagger}$ and Yaobo Zhang $\oplus^{1, \$}$ \\ ${ }^{1}$ Zhejiang Institute of Modern Physics, Zhejiang University, \\ Hangzhou 310027, People's Republlic of China \\ ${ }^{2}$ Center of Mathematical Science, Zhejiang University, Hangzhou 310027, People's Republic of China \\ ${ }^{3}$ Peng Huanwu Center for Fundamental Theory, Hefei, Anhui 230026, China \\ ${ }^{4}$ Beijing Computational Science Research Center, Beijing 100084, China \\ ${ }^{5}$ Hangzhou Institute for Advanced study, UCAS, Hongzhou 310024, China
}

(Received 20 October 2021; accepted 8 November 2021; published 24 February 2022)

\begin{abstract}
In the $\mathrm{CHY}$-frame for the amplitudes, there are two kinds of singularities we need to deal with. The first one is the pole singularities when the kinematics is not general, such that some of $S_{A} \rightarrow 0$. The second one is the collapse of locations of points after solving scattering equations (i.e., the singular solutions). These two types of singularities are tightly related to each other, but the exact mapping is not well understood. In this paper, we have initiated the systematic study of the mapping. We have demonstrated the different mapping patterns using three typical situations, i.e., the factorization limit, the soft limit and the forward limit.
\end{abstract}

DOI: 10.1103/PhysRevD.105.036015

\section{MOTIVATION}

In past years, there are huge processes in the effective computation and deep understanding of scattering amplitudes using the so-called "on-shell program". Among these on-shell methods, the CHY-formalism [6-10] provides a fantastic angle to study scattering amplitudes. In the CHY-formalism, the tree-level amplitude is given by

$\mathcal{A}_{n}=\int \frac{\left(\prod_{i=1}^{n} d z_{i}\right)}{\operatorname{vol}(S L(2, \mathbb{C}))} \Omega(\mathcal{E}) \mathcal{F}(z)=\int \frac{\left(\prod_{i=1}^{n} d z_{i}\right)}{d \omega} \Omega(\mathcal{E}) \mathcal{F}$,

where the integration is done over $z_{i}$ 's, which describe the locations of $n$-external particles living on $\mathbb{C P}^{1}$. Although there are $n$ variables, because the Möbius $S L(2, \mathbb{C})$ symmetry, three variables should be fixed, thus we need to divide the $d \omega=\frac{d z_{r} d z_{s} d z_{t}}{z_{r s} z_{s t} z_{t r}}$ with $z_{i j}=z_{i}-z_{j}$ by the gauge fixing. The $\Omega(\mathcal{E})$ is given by

$$
\Omega(\mathcal{E}) \equiv z_{i j} z_{j k} z_{k i} \prod_{a \neq i, j, k} \delta\left(\mathcal{E}_{a}\right),
$$

*fengbo@zju.edu.cn

isiahalbert@126.com

*yaobozhang@zju.edu.cn

${ }^{1}$ See for example, the tree-level on-shell recursion relation $[1,2]$ and one-loop unitarity cut method [3-5].

Published by the American Physical Society under the terms of the Creative Commons Attribution 4.0 International license. Further distribution of this work must maintain attribution to the author(s) and the published article's title, journal citation, and DOI. Funded by SCOAP ${ }^{3}$. where $\mathcal{E}_{a}$ 's are the scattering equations defined as

$$
\mathcal{E}_{a} \equiv \sum_{b \neq a} \frac{S_{a b}}{z_{a}-z_{b}}=0, \quad a=1,2, \ldots, n,
$$

with $S_{a b}=\left(k_{a}+k_{b}\right)^{2}=2 k_{a} \cdot k_{b}$, and $k_{a}, a=1,2, \ldots, n$ as $n$ massless momenta for $n$-external particles. The $\mathcal{F}$ is called the CHY-integrand, which defines the particular theory we are considering. Since there are $(n-3)$ delta-functions for $(n-3)$ variables, there is, in fact, no integration to be done in (1.1) and amplitudes are calculated as

$$
\mathcal{A}_{n}=\sum_{\text {sol }} \frac{z_{i j} z_{j k} z_{k i} z_{r s} z_{s t} z_{t r}}{(-)^{i+j+k+r+s+t}|\Phi|_{i j k}^{r s t}} \mathcal{F}
$$

where three arbitrary indices $i, j, k$ correspond to three removed scattering equations while three arbitrary indices $r, s, t$ correspond to three fixed locations mentioned above. The sum is over the solution set of the scattering equations, which is generically a discrete set of points. Furthermore, in the above, the Jacobi matrix $\Phi$ is calculated as ( $a$ for rows and $b$ for column)

$$
\Phi_{a b}=\frac{\partial \mathcal{E}_{a}}{\partial z_{b}}= \begin{cases}\frac{S_{a b}}{z_{a b}^{2}} & a \neq b \\ -\sum_{c \neq a} \frac{S_{a c}}{z_{a c}^{2}} & a=b\end{cases}
$$


and $|\Phi|_{i j k}^{r s t}$ is the determinant of $\Phi$ after removing the $i$ th, $j$ th, and $k$ th rows and $r$ th, $s$ th, and $t$ th columns.

From the above brief introduction, one can see that unlike the familiar off-shell Lagrangian formalism where the Feynman diagrams give a very intuitive picture to see the interaction by vertexes and propagators, all physical information in the CHY-formalism is coded by universal scattering equations and special rational $\mathrm{CHY}$-integrand of a given theory. Thus it is crucial to understand how these two total different descriptions are mapped to each other. For example, the general tree-level amplitudes have the factorization property, easily seen from the Feynman diagrams when putting a particular propagator on-shell. How do we see this in the CHY-formalism? Since it is a general property, it cannot depend on $\mathcal{F}$, and thus from (1.4), the information must be coded in the solutions of scattering equations.

There are many works on studying scattering equations, and their solutions [11-30]. By these studies, now we have an obvious picture about the factorization property in the CHY-formalism. As shown in [13], if all $S_{A} \neq 0$, the values of the $z_{a}$ 's are distinct (i.e., $z_{i} \neq z_{j}, \forall i, j$ ) in every solution for all $(n-3)$ ! solutions of scattering equations. However, suppose there is one and only one $S_{A}=0$, which corresponds to a particular factorization channel. In that case, we will find that there are $\left(n_{A}-2\right) ! \times\left(n-n_{A}-2\right)$ ! solutions [8], where $z_{i}=z_{0}, \forall i \in A$. $^{2}$ For later convenience, we will call a solution "regular" if all $z_{i}$ 's are distinct and a solution to be "singular" if some $z_{i}$ collapse to the same value. Using this terminology, one can say the solution singularity reflects that pole singularities. The understanding of factorization in the CHY-formalism is crucial because, through its study, the "integration rule" for simple poles and its generalization for higher poles have been proposed [31-33] and we can read out the analytic expression for any CHY-integrand without solving the scattering equations. One crucial concept coming out from the "integration rule" is the pole index

$$
\chi(A):=\mathbb{L}[A]-2(|A|-1)
$$

where $\mathbb{L}[A]$ be the number ${ }^{3}$ of lines connecting these nodes inside $A$ and $|A|$ is the number of nodes. The condition

$$
\chi(A) \geq 0
$$

will be called the pole condition for a given subset. More explicitly, each subset gives a possible nonzero pole

\footnotetext{
${ }^{2}$ In fact, because the gauge fixing of three $z_{i}$ 's, between the two subsets of $A, \bar{A}$, it is the one containing at most one gauge fixing point having $z_{i}=z_{0}$.

${ }^{3}$ Again more accurately it is the difference of number between solid lines and dashed lines, which represent the factor $z_{i j}=$ $z_{i}-z_{j}$ in the denominator or the numerator respectively.
}

contribution when and only when $\chi(A) \geq 0$, and the pole will be $\frac{1}{S_{A}^{(A)+1}}$, where $S_{A}=\left(p_{a_{1}}+p_{a_{2}}+\cdots+p_{a_{m}}\right)^{2}$ for massless momentum $p_{i}{ }^{4}$

Factorization singularity is just one type of singularity of tree-level amplitudes. There are other types of singularities, for example, soft singularity and forward singularity. In fact, forward singularity appearances naturally in the construction of loop-level CHY-formalism and dealing with it is important for the whole construction [15,25,34-53]. Different from factorization singularity, where we can arrange kinematics so there is one and only one $S_{A}=0$, both soft and forward limits will have multiple poles going to zero simultaneously. For example, if $k_{n} \rightarrow 0$, we will have all $S_{n i} \rightarrow 0, i=1, \ldots, n-1$. Similarly, for forward limit $k_{+}=-k_{-}$, we will have all $\left(k_{+}+k_{-}+k_{i}\right)^{2} \rightarrow 0$. It is natural to ask what is the solution behavior under these limits. For forward limit, beautiful analysis has been done in [53]. Motivated by these observations, in this paper, we will start a systematical discussion about the relation of the following two things: the kinematic singularities and the singular solutions of scattering equations. More explicitly, at one side, starting from the singular kinematic configuration with one or more singular poles, we will ask:

(i) (A1) If there will be singular solutions of scattering equations?

(ii) (A2) If there are, how many singular solutions?

(iii) (A3) What is the exact behavior of these singular solutions? Are all of them have similar behavior or different patterns?

(iv) (A4) What are the contributions of these singular solutions when putting them into CHY-integrands?

(v) (A5) Do regular solutions contribute to a singular part of scattering amplitudes?

On another side, starting from a given singular solution, we will ask:

(i) (B1) Is there a singular kinematic configuration implied by the solution?

(ii) (B2) If there are, how many different singular kinematic configurations? Are these poles compatible or not compatible?

Although we cannot answer the above questions in this paper, we will illustrate cases where two side problems can be theoretically analyzed. Besides theoretical analysis, we also provide numerical checking to support our arguments.

The plan of the paper is as follows. In. Sec. II, we have discussed from singular solutions what is the kinematic configuration we can infer. From Sec. III-V, we use factorization limit, soft limit and forward limit to demonstrate how to get pieces of information of singular solutions from the singular kinematics. Finally, a summary is given

\footnotetext{
${ }^{4}$ Because the momentum conservation, we will treat the subset $A$ to be equivalent to its complement $\bar{A}=\{1,2, \ldots, n\}-A$ when taking the pole. However, as we will show, the behavior of solutions will be very different between $A, \bar{A}$.
} 
in Sec. VI. In this paper, to better understand the theoretical analysis, we have offered several numerical computations. In the Appendix, how to implement the numerical kinematic configuration has been carefully discussed.

\section{FROM SINGULAR SOLUTIONS TO KINEMATICS}

As discussed in the previous section, we will discuss relations between singular solutions and kinematics in this note. This section will assume the behavior of singular solutions and derive the corresponding consequence of the kinematics, especially the pole structure. Let us consider an asymptotic behavior of the kinematics $k_{i}$ 's such that when $\epsilon \rightarrow 0$, the kinematics will reach the one we want:

$$
k_{i}=\sum_{a=0} \epsilon^{a} k_{i}^{(a)} .
$$

With this asymptotic behavior, solutions $z_{i}$ have also asymptotic behavior like:

$$
z_{i}=\sum_{a=0} \epsilon^{a} z_{i}^{(a)}
$$

Singular solutions will correspond to $z_{i}^{(0)} \rightarrow z_{S}$ for some $i \in S$ where the subset $S$ has at least two elements.

A first important observation made in [13] is that if there is at least one singular solution, then at least one kinematic pole goes to zero. The argument in [13] is following. Assuming there is at least one singular solution in (2.2) satisfying following condition:

Cond $\mathrm{A}: z_{i}^{(0)}=z_{S}, \quad a \in S ; \quad z_{a b}^{(0)} \neq 0$,

$z_{b \tilde{b}}^{(0)} \neq 0, \quad \forall a \in S, b, \tilde{b} \notin S$.

Putting this back, the scattering equations (1.3) will be factorized into two parts. For $a \in S$, we will have

$$
\begin{aligned}
\mathcal{E}_{a} & =\sum_{t \neq a} \frac{k_{a} \cdot k_{t}}{z_{a}-z_{t}}=\sum_{t \neq a, t \in S} \frac{k_{a} \cdot k_{t}}{z_{a}-z_{t}}+\sum_{t \notin S} \frac{k_{a} \cdot k_{t}}{z_{a}-z_{t}} \\
& =\frac{1}{\epsilon}\left\{\sum_{t \neq a, t \in S} \frac{\mathcal{K}_{a t}^{(00)}}{z_{a t}^{(1)}}\right\}+\left\{\sum_{t \neq a, t \in S} \frac{\mathcal{K}_{a t}^{(01)}+\mathcal{K}_{a t}^{(10)}}{z_{a t}^{(1)}}+\sum_{t \neq a, t \in S} \frac{-\left(z_{a t}^{(2)}\right) \mathcal{K}_{a t}^{(00)}}{\left(z_{a t}^{(1)}\right)^{2}}+\sum_{t \notin S} \frac{\mathcal{K}_{a t}^{(00)}}{z_{a t}^{(0)}}\right\}+\mathcal{O}(\epsilon)
\end{aligned}
$$

where for simplicity we have defined $z_{i j}^{(a)} \equiv z_{i}^{(a)}-z_{j}^{(a)}$ and $\mathcal{K}_{i j}^{(a b)} \equiv k_{i}^{(a)} \cdot k_{j}^{(b)}$, while for $a \notin S$, we have

$$
\mathcal{E}_{a}=\sum_{t \neq a, t \notin S} \frac{k_{a} \cdot k_{t}}{z_{a}-z_{t}}+\sum_{t \in S} \frac{k_{a} \cdot k_{t}}{z_{a}-z_{t}}=\sum_{t \neq a, t \notin S} \frac{\mathcal{K}_{a t}^{(00)}}{z_{a t}^{(0)}}+\sum_{t \in S} \frac{\mathcal{K}_{a t}^{(00)}}{z_{a}^{(0)}-z_{S}}+\mathcal{O}(\epsilon) .
$$

An important point is that the scattering equation should hold for each order of $\epsilon$, thus from the leading order of (2.4), we get the relation

$$
\mathcal{E}_{a ; \epsilon^{-1}} \equiv \sum_{t \neq a, t \in S} \frac{\mathcal{K}_{a t}^{(00)}}{z_{a t}^{(1)}}=0
$$

Using this result, we can see

$$
\begin{aligned}
\left(K_{S}^{(0)}\right)^{2} & \equiv\left(\sum_{a \in S} k_{a}^{(0)}\right)^{2}=\sum_{\substack{a, b \in S \\
a<b}} 2 k_{a}^{(0)} \cdot k_{b}^{(0)}=\sum_{\substack{a, b \in S \\
a<b}} \frac{z_{a}^{(1)}-z_{b}^{(1)}-z_{b}^{(1)}}{(1)} k_{a}^{(0)} \cdot k_{b}^{(0)} \\
& =\sum_{\substack{a, b \in S \\
a \neq b}} \frac{z_{a}^{(1)}}{z_{a}^{(1)}-z_{b}^{(1)}} 2 k_{a}^{(0)} \cdot k_{b}^{(0)}=2 \sum_{a \in S} z_{a}^{(1)} \sum_{\substack{b \in S \\
b \neq a}} \frac{k_{a}^{(0)} \cdot k_{b}^{(0)}}{z_{a}^{(1)}-z_{b}^{(1)}}=2 \sum_{a \in S} z_{a}^{(1)} \mathcal{E}_{a ; \epsilon^{-1}}=0 .
\end{aligned}
$$

In other words, assuming the singular solution with behavior (2.3) we have derived the kinematic information $\left(K_{S}^{(0)}\right)^{2}=0$.

The above derivation is beautiful, but if one checks it carefully, one can find a hidden assumption having been made, i.e., all $z_{a}^{(1)}-z_{b}^{(1)} \neq 0$ for $a, b \in S$. In other words, points in the subset $S$ going to the same location with the same order of speed, 
i.e., there is no subset such that points of this subset collapse to each other faster. If there is a subset violating the assumption, the leading term in (2.4) will be at least the order of $\frac{1}{\epsilon^{2}}$ and the conclusion will be modified. With this in mind, we consider various generalizations of the above derivation with different singular solution behaviors.

\section{A. The first case}

Let us start with the simplest generalization. Again, let us assume there is at least one singular solution satisfying the condition A given in (2.3). However, for these points going together, a subset of points collapses to each other faster, i.e., they satisfy one more condition.

$$
\begin{aligned}
& \text { Cond B : } z_{i}^{(1)}=z_{S_{1}}\left(\text { i.e, } z_{a \tilde{a}}^{(1)}=0\right), \quad z_{a \tilde{a}}^{(2)} \neq 0 \quad \forall a, \tilde{a} \in S_{1} \subset S \text {; } \\
& z_{a b}^{(1)} \neq 0, \quad z_{b \tilde{b}}^{(1)} \neq 0, \quad \forall a \in S_{1}, b, \tilde{b} \in S \backslash S_{1} .
\end{aligned}
$$

With these two conditions, the scattering equation (2.5) is not modified, but Eq. (2.4) should be refined as

$$
\begin{aligned}
\mathcal{E}_{a} & =\sum_{t \neq a, t \in S_{1}} \frac{k_{a} \cdot k_{t}}{z_{a}-z_{t}}+\sum_{t \in S \backslash S_{1}} \frac{k_{a} \cdot k_{t}}{z_{a}-z_{t}}+\sum_{t \notin S} \frac{k_{a} \cdot k_{t}}{z_{a}-z_{t}} \\
& =\frac{1}{\epsilon^{2}}\left\{\sum_{t \neq a, t \in S_{1}} \frac{\mathcal{K}_{a t}^{(00)}}{z_{a t}^{(2)}}\right\}+\frac{1}{\epsilon}\left\{\sum_{t \neq a, t \in S_{1}} \frac{\mathcal{K}_{a t}^{(01)}+\mathcal{K}_{a t}^{(10)}}{z_{a t}^{(2)}}+\sum_{t \neq a, t \in S_{1}} \frac{-\left(z_{a t}^{(3)}\right) \mathcal{K}_{a t}^{(00)}}{\left(z_{a t}^{(2)}\right)^{2}}+\sum_{t \in S \backslash S_{1}} \frac{\mathcal{K}_{a t}^{(00)}}{z_{a t}^{(1)}}\right\}+\mathcal{O}\left(\epsilon^{0}\right)
\end{aligned}
$$

when $a \in S_{1}$ and

$$
\begin{aligned}
\mathcal{E}_{a} & =\sum_{t \neq a, t \in S \backslash S_{1}} \frac{k_{a} \cdot k_{t}}{z_{a}-z_{t}}+\sum_{t \in S_{1}} \frac{k_{a} \cdot k_{t}}{z_{a}-z_{t}}+\sum_{t \notin S} \frac{k_{a} \cdot k_{t}}{z_{a}-z_{t}} \\
& =\frac{1}{\epsilon}\left\{\sum_{t \neq a, t \in S} \frac{\mathcal{K}_{a t}^{(00)}}{z_{a t}^{(1)}}\right\}+\left\{\sum_{t \neq a, t \in S} \frac{\mathcal{K}_{a t}^{(01)}+\mathcal{K}_{a t}^{(10)}}{z_{a t}^{(1)}}+\sum_{t \neq a, t \in S} \frac{-\left(z_{a t}^{(2)}\right) \mathcal{K}_{a t}^{(00)}}{\left(z_{a t}^{(1)}\right)^{2}}+\sum_{t \notin S} \frac{\mathcal{K}_{a t}^{(00)}}{z_{a t}^{(0)}}\right\}+\mathcal{O}(\epsilon)
\end{aligned}
$$

when $a \in S \backslash S_{1}$. From the refined scattering equations (2.9) and (2.10), we obtain following identities

$$
\begin{gathered}
\mathcal{E}_{a \in S_{1} ; \epsilon^{-2}}=\sum_{t \neq a, t \in S_{1}} \frac{\mathcal{K}_{a t}^{(00)}}{z_{a t}^{(2)}}=0 \\
\mathcal{E}_{a \in S_{1} ; \epsilon^{-1}}=\sum_{t \neq a, t \in S_{1}} \frac{\mathcal{K}_{a t}^{(01)}+\mathcal{K}_{a t}^{(10)}}{z_{a t}^{(2)}}+\sum_{t \neq a, t \in S_{1}} \frac{-\left(z_{a t}^{(3)}\right) \mathcal{K}_{a t}^{(00)}}{\left(z_{a t}^{(2)}\right)^{2}}+\sum_{t \in S \backslash S_{1}} \frac{\mathcal{K}_{a t}^{(00)}}{z_{a t}^{(1)}} \\
\mathcal{E}_{a \in S \backslash S_{1} ; \epsilon^{-1}}=\sum_{t \neq a, t \in S} \frac{\mathcal{K}_{a t}^{(00)}}{z_{a t}^{(1)}}=\sum_{t \in S_{1}} \frac{\mathcal{K}_{a t}^{(00)}}{z_{a t}^{(1)}}+\sum_{t \neq a, t \in S \backslash S_{1}} \frac{\mathcal{K}_{a t}^{(00)}}{z_{a t}^{(1)}} \\
\mathcal{E}_{a \in S \backslash S_{1} ; \epsilon^{0}}=\sum_{t \neq a, t \in S} \frac{\mathcal{K}_{a t}^{(01)}+\mathcal{K}_{a t}^{(10)}}{z_{a t}^{(1)}}+\sum_{t \neq a, t \in S} \frac{-\left(z_{a t}^{(2)}\right) \mathcal{K}_{a t}^{(00)}}{\left(z_{a t}^{(1)}\right)^{2}}+\sum_{t \notin S} \frac{\mathcal{K}_{a t}^{(00)}}{z_{a t}^{(0)}} .
\end{gathered}
$$

Now mimicking the previous argument, first using (2.11) one can show

$$
\begin{aligned}
\left(k_{S_{1}}^{(0)}\right)^{2} \equiv\left(\sum_{a \in S_{1}} k_{a}^{(0)}\right)^{2} & =\sum_{\substack{a, b \in S_{1} \\
a<b}} 2 k_{a}^{(0)} \cdot k_{b}^{(0)}=\sum_{\substack{a, b \in S_{1} \\
a<b}} \frac{z_{a}^{(2)}-z_{b}^{(2)}}{z_{a}^{(2)}-z_{b}^{(2)}} 2 k_{a}^{(0)} \cdot k_{b}^{(0)} \\
& =\sum_{\substack{a, b \in S_{1} \\
a \neq b}} \frac{z_{a}^{(2)}}{z_{a}^{(2)}-z_{b}^{(2)}} 2 k_{a}^{(0)} \cdot k_{b}^{(0)}=2 \sum_{a \in S_{1}} z_{a}^{(2)} \mathcal{E}_{a \in S_{1} ; \epsilon^{-2}}=0
\end{aligned}
$$


Second, let us consider following combination

$$
\begin{aligned}
2 K_{S_{1}} \cdot K_{S \backslash S_{1}}+K_{S \backslash S_{1}}^{2} & =\sum_{a \in S_{1}, b \in S \backslash S_{1}} 2 k_{a}^{(0)} \cdot k_{b}^{(0)}+\sum_{b, \tilde{b} \in S \backslash S_{1}, b<\tilde{b}} 2 k_{b}^{(0)} \cdot k_{\tilde{b}}^{(0)} \\
& =\sum_{a \in S_{1}, b \in S \backslash S_{1}} \frac{z_{a b}^{(1)}}{z_{a b}^{(1)}} 2 k_{a}^{(0)} \cdot k_{b}^{(0)}+\sum_{b, \tilde{b} \in S \backslash S_{1}, b<\tilde{b}} \frac{\left(z_{b}^{(1)}-z_{S_{1}}\right)-\left(z_{\tilde{b}}^{(1)}-z_{S_{1}}\right)}{z_{b}^{(1)}-z_{\tilde{b}}^{(1)}} 2 k_{b}^{(0)} \cdot k_{\tilde{b}}^{(0)} \\
& \left.=\sum_{a \in S_{1}, b \in S \backslash S_{1}} \frac{\left(z_{b}^{(1)}-z_{S_{1}}\right)}{(1)}-z_{S_{1}}\right) \\
& =\sum_{b \in S \backslash S_{1}} 2\left(z_{b}^{(1)}-z_{S_{1}}\right)\left(\sum_{a \in S_{1}} \frac{1}{\left(z_{b}^{(1)}-z_{S_{1}}\right)} k_{a, \tilde{b} \in S \backslash S_{1}, b \neq \tilde{b}} \frac{\left(z_{b}^{(1)}-z_{S_{1}}\right)}{z_{b}^{(1)}-z_{\tilde{b}}^{(1)}} 2 k_{b}^{(0)} \cdot k_{\tilde{b}}^{(0)}+\sum_{\tilde{b} \in S \backslash S_{1}, b \neq \tilde{b}} \frac{1}{z_{b}^{(1)}-z_{\tilde{b}}^{(1)}} k_{b}^{(0)} \cdot k_{\tilde{b}}^{(0)}\right) \\
& =\sum_{b \in S \backslash S_{1}} 2\left(z_{b}^{(1)}-z_{S_{1}}\right) \mathcal{E}_{b \in S \backslash S_{1} ; \varepsilon^{-1}}=0
\end{aligned}
$$

Combining (2.15) and (2.16) we have

$$
\begin{aligned}
& \left(k_{S}^{(0)}\right)^{2}=\sum_{\substack{a, b \in S_{1} \\
a<b}} 2 k_{a}^{(0)} \cdot k_{b}^{(0)}+\sum_{a \in S_{1}, b \in S \backslash S_{1}} 2 k_{a}^{(0)} \cdot k_{b}^{(0)} \\
& +\sum_{b, \tilde{b} \in S \backslash S_{1}, b<\tilde{b}} 2 k_{b}^{(0)} \cdot k_{\tilde{b}}^{(0)}=0 \\
& \epsilon^{0} \quad \epsilon^{1} \quad \epsilon^{2} \\
& z_{S \leftarrow} \overbrace{}^{z_{S_{1}}}\} S S_{1} \\
& \left.\begin{array}{c}
\bullet \\
\bullet
\end{array}\right\} \bar{S}
\end{aligned}
$$

FIG. 1. The pictorial representation of conditions A and B exhibited in (2.3) and (2.8). Since $z_{i \in \bar{S}}^{(0)}$ 's are different values, we represent them by a node under the column of $\epsilon^{0}$. For the subset $S$, they have the same $z_{i \in S}^{(0)}=z_{S}$, which is represented by a node with several lines under the column of $\epsilon^{0}$. Similarly, for the subset $S_{1}$, they have the same $z_{i \in S_{1}}^{(1)}=z_{S_{1}}$, which is represented by a node with several lines under the column of $\epsilon^{1}$. The ending of each line has meaning too. Different positions in a given column mean the different $z_{i}$ 's at the given order of $\epsilon$. For the subset $\bar{S}$, the ending under the column of $\epsilon^{0}$ means that all $z_{i \in \bar{S}}^{(0)}$ and $z_{S}$ are different, so the leading scattering equation is at the order of $\epsilon^{0}$ as given in (2.5). Similarly, for the subset $S \backslash S_{1}$, the ending under the column of $\epsilon^{1}$ means the leading scattering equation is at the order of $\epsilon^{1}$ as given in (2.13), while for the subset $S_{1}$, the ending under the column of $\epsilon^{2}$ means the leading scattering equation is at the order of $\epsilon^{2}$ as given in (2.11).
In other words, under the limit $\epsilon \rightarrow 0$, two poles go to zero at the same time.

The above case can be generalized to more configurations. To see that, let us first rephrase the condition A and B in (2.3) and (2.8) in Fig. 1. With the pictorial representation, we consider the more general one given in Fig. 2. We will call it the "Type A" structure, where each node has several branches, but at most, one branch has a substructure. From Fig. 2, we can read out the following kinematic information. First, using the leading order part (at the order $\epsilon^{-k}$ ) of scattering equations of the subset $S_{k}$.

$$
\mathcal{E}_{a \in S_{k} ; \epsilon^{-k}}=\sum_{t \neq a, t \in S_{k}} \frac{k_{a}^{(0)} \cdot k_{t}^{(0)}}{z_{a}^{(k)}-z_{t}^{(k)}}=0
$$

we can derive

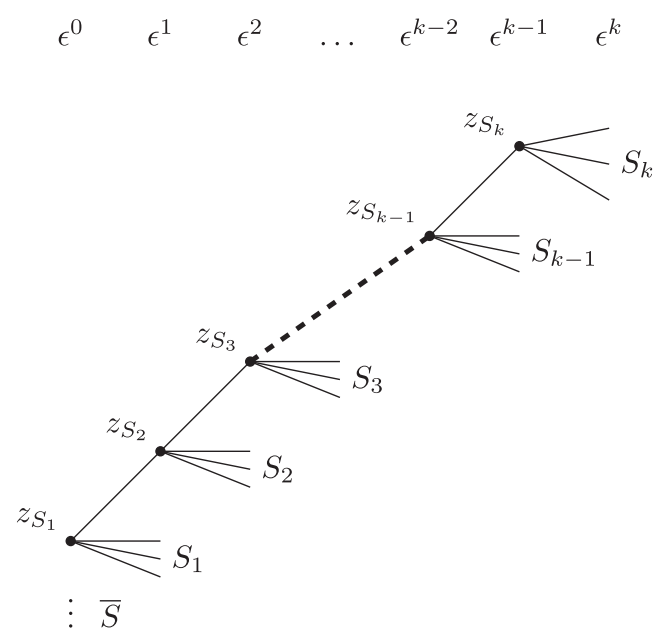

FIG. 2. The pictorial representation of the Type A generalization. For this type, where each node has several branches, but at most one branch has substructure. We have also used the $z$ to denote the common position at each order of $\epsilon$. 


$$
\begin{aligned}
\left(k_{S_{k}}^{(0)}\right)^{2} \equiv \sum_{\substack{a, b \in S_{k} \\
a<b}} 2 k_{a}^{(0)} \cdot k_{b}^{(0)} & =\sum_{\substack{a, b \in S_{k} \\
a \neq b}} \frac{z_{a}^{(k)}}{z_{a}^{(k)}-z_{b}^{(k)}} 2 k_{a}^{(0)} \cdot k_{b}^{(0)} \\
& =2 \sum_{a \in S_{k}} z_{a}^{(k)} \mathcal{E}_{a \in S_{1} ; \epsilon^{-k}}=0 .
\end{aligned}
$$

Next we consider the leading order part (at the order $\epsilon^{-(k-1)}$ ) of scattering equations of the subset $S_{k-1}$

$$
\begin{aligned}
\mathcal{E}_{a \in S_{k-1} ; \epsilon^{-(k-1)}} & =\sum_{t \in S_{k}} \frac{k_{a}^{(0)} \cdot k_{t}^{(0)}}{z_{a}^{(k-1)}-z_{S_{k}}}+\sum_{t \neq a, t \in S_{k-1}} \frac{k_{a}^{(0)} \cdot k_{t}^{(0)}}{z_{a}^{(k-1)}-z_{t}^{(k-1)}} \\
& =\frac{k_{a}^{(0)} \cdot K_{S_{k}}^{(0)}}{z_{a}^{(k-1)}-z_{S_{k}}}+\sum_{t \neq a, t \in S_{k-1}} \frac{k_{a}^{(0)} \cdot k_{t}^{(0)}}{z_{a}^{(k-1)}-z_{t}^{(k-1)}}=0 .
\end{aligned}
$$

Using it, we can show

$$
\begin{aligned}
2 K_{S_{k}} & K_{S_{k-1}}+K_{S_{k-1}}^{2} \\
= & 2 \sum_{a \in S_{k-1}} \frac{z_{a}^{(k-1)}-z_{S_{k}}}{z_{a}^{(k-1)}-z_{S_{k}}} k_{a}^{(0)} \cdot K_{S_{k}}^{(0)} \\
& +2 \sum_{t \neq a, t \in S_{k-1}} \frac{\left(z_{a}^{(k-1)}-z_{S_{k}}\right) k_{a}^{(0)} \cdot k_{t}^{(0)}}{z_{a}^{(k-1)}-z_{t}^{(k-1)}} \\
= & 2 \sum_{a \in S_{k-1}}\left(z_{a}^{(k-1)}-z_{S_{k}}\right) \mathcal{E}_{a \in S_{k-1} ; \epsilon^{-(k-1)}}=0 .
\end{aligned}
$$

When combining (2.19) and (2.21), we reach $K_{S_{k} \cup S_{k-1}}^{2}=0$. Similarly from the leading order part (at the order $\epsilon^{-(k-2)}$ ) of scattering equations of the subset $S_{k-2}$

$\mathcal{E}_{a \in S_{k-2} ; \epsilon^{-(k-2)}}=\frac{k_{a}^{(0)} \cdot K_{S_{k} \cup S_{k-1}}^{(0)}}{z_{a}^{(k-2)}-z_{S_{k-1}}}+\sum_{t \neq a, t \in S_{k-2}} \frac{k_{a}^{(0)} \cdot k_{t}^{(0)}}{z_{a}^{(k-2)}-z_{t}^{(k-2)}}=0$,

we can derive $2 K_{S_{k} \cup S_{k-1}} \cdot K_{S_{k-2}}+K_{S_{k-2}}^{2}=0$ and further $K_{S_{k} \cup S_{k-1} \cup S_{k-2}}^{2}=0$. Iterating the procedure, one can easily see that from the Fig. 2, we can derive

$$
K_{\mathrm{U}_{t=j}^{k} S_{t}}^{2}=0, \quad j=1, \ldots, k
$$

An important point of the above iterating procedure is that when going to the lower orders of $\epsilon$, see for example (2.22), the detail structure of the node $z_{S_{k-1}}$ does not appear. In other words, we can treat the subset $S_{k} \cup S_{k-1}$ as an effective single leg when considering the subset $S_{k-2}$.

\section{B. The second case}

In the first case, we have required that in the pictorial representation of solution $z_{i}$ 's, although each node has several branches, there is at most one branch having a substructure. Now we consider the case that there are at least two branches having substructures. For simplicity, let consider the situation given in Fig. 3. For $a \in S_{3}$, we have the scattering equations as

$$
\begin{aligned}
\mathcal{E}_{a \in S_{3}}= & \frac{1}{\epsilon^{2}}\left\{\sum_{t \neq a, t \in S_{3}} \frac{k_{a}^{(0)} \cdot k_{t}^{(0)}}{z_{a}^{(2)}-z_{t}^{(2)}}\right\} \\
& +\frac{1}{\epsilon}\left\{\sum_{t \neq a, t \in S_{3}} \frac{k_{a}^{(0)} \cdot k_{t}^{(1)}+k_{a}^{(1)} \cdot k_{t}^{(0)}}{z_{a}^{(2)}-z_{t}^{(2)}}\right. \\
& +\sum_{t \neq a, t \in S_{3}} \frac{-\left(z_{a}^{(3)}-z_{t}^{(3)}\right)\left(k_{a}^{(0)} \cdot k_{t}^{(0)}\right)}{\left(z_{a}^{(2)}-z_{t}^{(2)}\right)^{2}} \\
& \left.+\sum_{t \in S_{1}} \frac{k_{a}^{(0)} \cdot k_{t}^{(0)}}{z_{S_{3}}-z_{t}^{(1)}}+\sum_{t \in S_{2}} \frac{k_{a}^{(0)} \cdot k_{t}^{(0)}}{z_{S_{3}}-z_{S_{2}}}\right\}+\mathcal{O}\left(\epsilon^{0}\right)
\end{aligned}
$$

thus we get

$$
\begin{aligned}
& \mathcal{E}_{a \in S_{3} ; \epsilon^{-2}}=\sum_{t \neq a, t \in S_{3}} \frac{k_{a}^{(0)} \cdot k_{t}^{(0)}}{z_{a}^{(2)}-z_{t}^{(2)}}=0 \\
\mathcal{E}_{a \in S_{3} ; \epsilon^{-1}}= & \sum_{t \neq a, t \in S_{3}} \frac{k_{a}^{(0)} \cdot k_{t}^{(1)}+k_{a}^{(1)} \cdot k_{t}^{(0)}}{z_{a}^{(2)}-z_{t}^{(2)}} \\
& +\sum_{t \neq a, t \in S_{3}} \frac{-\left(z_{a}^{(3)}-z_{t}^{(3)}\right)\left(k_{a}^{(0)} \cdot k_{t}^{(0)}\right)}{\left(z_{a}^{(2)}-z_{t}^{(2)}\right)^{2}} \\
& +\sum_{t \in S_{1}} \frac{k_{a}^{(0)} \cdot k_{t}^{(0)}}{z_{S_{3}}-z_{t}^{(1)}}+\frac{k_{a}^{(0)} \cdot K_{S_{2}}^{(0)}}{z_{S_{3}}-z_{S_{2}}}=0 .
\end{aligned}
$$

Similarly, for $a \in S_{2}$ we get

$$
\mathcal{E}_{a \in S_{2} ; \epsilon^{-2}}=\sum_{t \neq a, t \in S_{2}} \frac{k_{a}^{(0)} \cdot k_{t}^{(0)}}{z_{a}^{(2)}-z_{t}^{(2)}}=0
$$

$$
\epsilon^{0} \quad \epsilon^{1} \quad \epsilon^{2}
$$

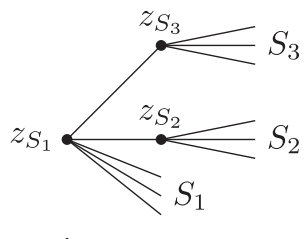

$\bar{S}$

FIG. 3. The pictorial representation of one simple case of the Type B, where a node has two branches having substructure. 


$$
\mathcal{E}_{a \in S_{2} ; \epsilon^{-1}}=\sum_{t \neq a, t \in S_{2}} \frac{k_{a}^{(0)} \cdot k_{t}^{(1)}+k_{a}^{(1)} \cdot k_{t}^{(0)}}{z_{a}^{(2)}-z_{t}^{(2)}}+\sum_{t \neq a, t \in S_{2}} \frac{-\left(z_{a}^{(3)}-z_{t}^{(3)}\right)\left(k_{a}^{(0)} \cdot k_{t}^{(0)}\right)}{\left(z_{a}^{(2)}-z_{t}^{(2)}\right)^{2}}+\sum_{t \in S_{1}} \frac{k_{a}^{(0)} \cdot k_{t}^{(0)}}{z_{S_{2}}-z_{t}^{(1)}}+\frac{k_{a}^{(0)} \cdot K_{S_{3}}^{(0)}}{z_{S_{2}}-z_{S_{3}}}=0 .
$$

For $a \in S_{1}$ we get

$$
\begin{aligned}
\mathcal{E}_{a \in S_{1} ; \epsilon^{-1}} & =\sum_{t \neq a, t \in S_{1}} \frac{k_{a}^{(0)} \cdot k_{t}^{(0)}}{z_{a}^{(1)}-z_{t}^{(1)}}+\sum_{t \in S_{2}} \frac{k_{a}^{(0)} \cdot k_{t}^{(0)}}{z_{a}^{(1)}-z_{S_{2}}}+\sum_{t \in S_{3}} \frac{k_{a}^{(0)} \cdot k_{t}^{(0)}-z_{S_{3}}}{z_{a}^{(1)}} \\
& =\sum_{t \neq a, t \in S_{1}} \frac{k_{a}^{(0)} \cdot k_{t}^{(0)}}{z_{a}^{(1)}-z_{t}^{(1)}}+\frac{k_{a}^{(0)} \cdot k_{S_{2}}^{(0)}}{z_{a}^{(1)}-z_{S_{2}}}+\frac{k_{a}^{(0)} \cdot k_{S_{3}}^{(0)}}{z_{a}^{(1)}-z_{S_{3}}}=0 .
\end{aligned}
$$

Now let us see which kinematic information can be derived using above identities. First, using (2.25) and (2.27) we get

$$
K_{S_{2}}^{2}=0, \quad K_{S_{3}}^{2}=0
$$

Second, using (2.26) and (2.28) we get

$$
\begin{aligned}
0 & =\sum_{a \in S_{3}} \mathcal{E}_{a \in S_{3} ; \epsilon^{-1}}=\sum_{t \in S_{1}} \frac{K_{S_{3}}^{(0)} \cdot k_{t}^{(0)}}{z_{S_{3}}-z_{t}^{(1)}}+\frac{K_{S_{3}}^{(0)} \cdot K_{S_{2}}^{(0)}}{z_{S_{3}}-z_{S_{2}}} \\
0 & =\sum_{a \in S_{2}} \mathcal{E}_{a \in S_{2} ; \epsilon^{-1}}=\sum_{t \in S_{1}} \frac{K_{S_{2}}^{(0)} \cdot k_{t}^{(0)}}{z_{S_{2}}-z_{t}^{(1)}}+\frac{K_{S_{2}}^{(0)} \cdot K_{S_{3}}^{(0)}}{z_{S_{2}}-z_{S_{3}}} .
\end{aligned}
$$

Now we consider

$$
\begin{aligned}
2 & K_{S_{2}}^{(0)} \cdot K_{S_{3}}^{(0)}+2 \sum_{a \in S_{1}}\left(K_{S_{3}}^{(0)} \cdot k_{a}^{(0)}+K_{S_{2}}^{(0)} \cdot k_{a}^{(0)}\right)+2 \sum_{t<a, t, a \in S_{1}} k_{a}^{(0)} \cdot k_{t}^{(0)} \\
& =2 \sum_{t<a, t, a \in S_{1}} \frac{\left(z_{a}^{(1)}-z_{S_{2}}\right)-\left(z_{t}^{(1)}-z_{S_{2}}\right)}{z_{a}^{(1)}-z_{t}^{(1)}} k_{a}^{(0)} \cdot k_{t}^{(0)}+2 \sum_{a \in S_{1}} \frac{\left(z_{a}^{(1)}-z_{S_{2}}\right)}{\left(z_{a}^{(1)}-z_{S_{2}}\right)} K_{S_{2}}^{(0)} \cdot k_{a}^{(0)} \\
& +2 \sum_{a \in S_{1}} \frac{\left(z_{a}^{(1)}-z_{S_{2}}\right)+\left(z_{S_{2}}-z_{S_{3}}\right)}{\left(z_{a}^{(1)}-z_{S_{3}}\right)} K_{S_{3}}^{(0)} \cdot k_{a}^{(0)}+2 K_{S_{2}}^{(0)} \cdot K_{S_{3}}^{(0)} \\
= & 2 \sum_{a \in S_{1}}\left(z_{a}^{(1)}-z_{S_{2}}\right) \sum_{t \neq a, t \in S_{1}} \frac{k_{a}^{(0)} \cdot k_{a}^{(0)}-z_{t}^{(1)}}{z^{(1)}}+2 \sum_{a \in S_{1}}\left(z_{a}^{(1)}-z_{S_{2}}\right) \frac{K_{S_{2}}^{(0)} \cdot k_{a}^{(0)}}{\left(z_{a}^{(1)}-z_{S_{2}}\right)} \\
& +2 \sum_{a \in S_{1}}\left(z_{a}^{(1)}-z_{S_{2}}\right) \frac{K_{S_{3}}^{(0)} \cdot k_{a}^{(0)}}{\left(z_{a}^{(1)}-z_{S_{3}}\right)}+2\left(z_{S_{2}}-z_{S_{3}}\right) \sum_{a \in S_{1}} \frac{\left.K_{S_{3}}^{(0)} \cdot k_{a}^{(0)}-z_{S_{3}}\right)}{\left(z_{a}\right.}+2 K_{S_{2}}^{(0)} \cdot K_{S_{3}}^{(0)} \\
= & 2 \sum_{a \in S_{1}}\left(z_{a}^{(1)}-z_{S_{2}}\right) \mathcal{E}_{a \in S_{1} ; \epsilon^{-1}}=0
\end{aligned}
$$

where (2.31) has been used to reach the last line. When combining (2.33) with (2.30) we arrive at

$$
K_{S_{1} \cup S_{2} \cup S_{3}}^{2}=0 .
$$


We can generalize the above argument for more than two branches having substructure. Let us assume that (1) when $i \in S, z_{i}^{(0)}=z_{0}$; (2) when $i \in S_{j} \subset S, z_{i}^{(1)}=z_{S_{j}}$ with $j=1, \ldots, m$; (3) $S=S_{0} \cup S_{1} \cup \ldots \cup S_{m}$. For each $a \in S_{j}, j=1, \ldots, m$, we have following two identities

$$
\begin{aligned}
& \mathcal{E}_{a \in S_{j} ; \epsilon^{-2}}=\sum_{t \neq a, t \in S_{j}} \frac{k_{a}^{(0)} \cdot k_{t}^{(0)}-z_{t}^{(2)}}{z_{a}^{(2)}}=0 \\
\mathcal{E}_{a \in S_{j} ; \epsilon^{-1}}= & \sum_{t \neq a, t \in S_{j}} \frac{k_{a}^{(0)} \cdot k_{t}^{(1)}+k_{a}^{(1)} \cdot k_{t}^{(0)}}{z_{a}^{(2)}-z_{t}^{(2)}} \\
& +\sum_{t \neq a, t \in S_{j}} \frac{-\left(z_{a}^{(3)}-z_{t}^{(3)}\right)\left(k_{a}^{(0)} \cdot k_{t}^{(0)}\right)}{\left(z_{a}^{(2)}-z_{t}^{(2)}\right)^{2}} \\
& +\sum_{t \in S_{0}} \frac{k_{a}^{(0)} \cdot k_{t}^{(0)}}{z_{S_{j}}-z_{t}^{(1)}}+\sum_{p=1, p \neq j}^{m} \frac{k_{a}^{(0)} \cdot K_{S_{p}}^{(0)}}{z_{S_{j}}-z_{S_{p}}}=0
\end{aligned}
$$

and using them we get

$$
\begin{aligned}
& 0=K_{S_{j}}^{2}, \\
& 0=\sum_{a \in S_{j}} \mathcal{E}_{a \in S_{j} ; \epsilon^{-1}}=\sum_{t \in S_{0}} \frac{K_{S_{j}}^{(0)} \cdot k_{t}^{(0)}}{z_{S_{j}}-z_{t}^{(1)}}+\sum_{p=1, p \neq j}^{m} \frac{K_{S_{j}}^{(0)} \cdot K_{S_{p}}^{(0)}}{z_{S_{j}}-z_{S_{p}}}
\end{aligned}
$$

For $a \in S_{0}$, the leading part of scattering equations is

$$
\begin{aligned}
\mathcal{E}_{a \in S_{0} ; \epsilon^{-1}} & =\sum_{t \neq a, t \in S_{0}} \frac{k_{a}^{(0)} \cdot k_{t}^{(0)}}{z_{a}^{(1)}-z_{t}^{(1)}}+\sum_{j=1}^{m} \sum_{t \in S_{j}} \frac{k_{a}^{(0)} \cdot k_{t}^{(0)}}{z_{a}^{(1)}-z_{S_{j}}} \\
& =\sum_{t \neq a, t \in S_{0}} \frac{k_{a}^{(0)} \cdot k_{t}^{(0)}}{z_{a}^{(1)}-z_{t}^{(1)}}+\sum_{j=1}^{m} \frac{k_{a}^{(0)} \cdot K_{S_{j}}^{(0)}}{z_{a}^{(1)}-z_{S_{j}}}=0 .
\end{aligned}
$$

One important point of expressions (2.37) and (2.38) is that the subset $S_{j}$ has been effectively treated as a single leg. This fact is very useful to see in an arbitrary tree graph, how the computation done explicitly here is generalized. Using the above results, we can see

$$
\begin{aligned}
\Sigma & \equiv \sum_{1 \leq i<j \leq m} K_{S_{i}}^{(0)} \cdot K_{S_{j}}^{(0)}+\sum_{j=1}^{m} \sum_{t \in S_{0}} K_{S_{j}}^{(0)} \cdot k_{t}^{(0)}+\sum_{t<a \mid a, t \in S_{0}} k_{a}^{(0)} \cdot k_{t}^{(0)} \\
& =\sum_{t<a \mid a, t \in S_{0}} \frac{z_{a}^{(1)}-z_{t}^{(1)}}{z_{a}^{(1)}-z_{t}^{(1)}} k_{a}^{(0)} \cdot k_{t}^{(0)}+\sum_{1 \leq i<j \leq m} \frac{z_{S_{i}}-z_{S_{j}}}{z_{S_{i}}-z_{S_{j}}} K_{S_{i}}^{(0)} \cdot K_{S_{j}}^{(0)}+\sum_{j=1}^{m} \sum_{a \in S_{0}} \frac{z_{a}^{(1)}-z_{S_{j}}}{z_{a}^{(1)}-z_{S_{j}}} K_{S_{j}}^{(0)} \cdot k_{a}^{(0)} \\
& =\sum_{t \neq a ; a, t \in S_{0}} \frac{z_{a}^{(1)}}{z_{a}^{(1)}-z_{t}^{(1)}} k_{a}^{(0)} \cdot k_{t}^{(0)}+\sum_{i \neq j} \frac{z_{S_{i}}}{z_{S_{i}}-z_{S_{j}}} K_{S_{i}}^{(0)} \cdot K_{S_{j}}^{(0)}+\sum_{j=1}^{m} \sum_{a \in S_{0}} \frac{z_{a}^{(1)}-z_{S_{j}}}{(1)} K_{S_{j}}^{(0)} \cdot k_{a}^{(0)} \\
& =\sum_{a \in S_{0}} z_{a}^{(1)}\left\{\sum_{t \neq a, t \in S_{0}} \frac{k_{a}^{(0)} \cdot k_{t}^{(1)}-z_{t}^{(1)}}{z_{a}}+\sum_{j=1}^{m} \frac{K_{S_{j}}^{(0)} \cdot k_{a}^{(0)}}{z_{a}^{(1)}-z_{S_{j}}}\right\}+\sum_{j=1}^{m} z_{S_{j}}\left\{\sum_{a \in S_{0}} \frac{K_{S_{j}}^{(0)} \cdot k_{a}^{(0)}}{z_{S_{j}}-z_{a}^{(1)}}+\sum_{i=1, i \neq j}^{m} \frac{K_{S_{j}}^{(0)} \cdot K_{S_{i}}^{(0)}}{z_{S_{j}}-z_{S_{i}}}\right\} \\
& =0,
\end{aligned}
$$

where (2.38) and (2.37) have been used to reach the last line. When combining (2.39) and (2.37) we get

$$
K_{S}^{2}=0
$$

Summary: Although we have considered only a tree graph with two levels for type $\mathrm{B}$, it is easy to see combining results for type A and $\mathrm{B}$. The above observation can be easily generalized to a tree graph with an arbitrary structure. The basic conclusion is that for each node in the tree graph, we have $\left(\sum_{i \in \text { node }} k_{i}\right)^{2}=0$. One important remark is that for all subsets $S_{i}$ with $K_{S_{i}}^{2}=0$, they are compatible to each others, i.e., either $S_{i} \cap S_{j}=\varnothing$ or $S_{i} \subset S_{j}$.
With results in this section, one can also show that if $k_{S}^{2} \neq 0$ for all subsets $S$ with between 2 and $(N-2)$ elements, the values of the $z_{a}, a \in A$, are distinct, i.e., there are no singular solutions. This claim is proved in [13] by contradiction. Assuming there is a singular solution, by analysis in this section, we will reach at least one $S_{A} \rightarrow 0$ no matter which configuration of the singular solution is.

\section{FROM SINGULAR KINEMATICS TO SOLUTIONS I: THE FACTORIZATION LIMIT}

Starting from this section, we discuss what we can learn about the solutions of scattering equations when there are one or more poles going to on-shell. 


\section{A. With only one $S_{A} \rightarrow 0$}

This case has been well studied in $[8,13]$. Under the limit $\epsilon \rightarrow 0$, assuming there is one and only one $S_{A} \rightarrow 0$, what we can say about the solutions of scattering equations? Under the limit, we could have two possibilities: (R1) There is still no singular solution; (R2) There are some singular solutions.

Let us argue that the possibility (R1) could not be true first. We could assign a particular CHY-integrand, such as the multiplication of two PT-factors, which produces amplitude containing one and only one cubic Feynman diagram having the pole $S_{A}$. Since $S_{A} \rightarrow 0$, the amplitude will be divergent. Now let us check the expression (1.4) for the amplitude with gauge choice $(i j k)=(r s t)$ and three fixed points at the finite locations. We see that the numerator $\left(z_{i j} z_{j k} z_{k i}\right)^{2}$ is not divergent, as well as the factor $\frac{1}{|\Phi|_{i j k}^{\mid r s t}}$ and the particular CHY-integrand if the possibility (R1) is true. Thus by the contradiction, we see that the possibility (R1) could not be true.

Now the possibility (R2) is realized, and we discuss the properties of singular solutions. For a given singular solution, from the careful analysis in section two, we see that (a) the singular set can be and only be the subset $A^{5}$; (b) there is no substructure in $A$, i.e., every point in $A$ going to the same location with the same speed. If (a) is not true, we will have $S_{B} \rightarrow 0$ with $B \neq A$. If (b) is not true, we will have other $S_{B} \rightarrow 0$ with $B \subset A$. In fact more information is given in [8]: the number of singular solution is $\left(n_{A}+1-3\right) ! \times\left(n_{\bar{A}}+1-3\right) !$ where $n_{A}, n_{\bar{A}}$ are the number of elements in the subsets $A, \bar{A}$. The counting of the number of singular solutions can be easily seen from the left picture given in Fig. 4.
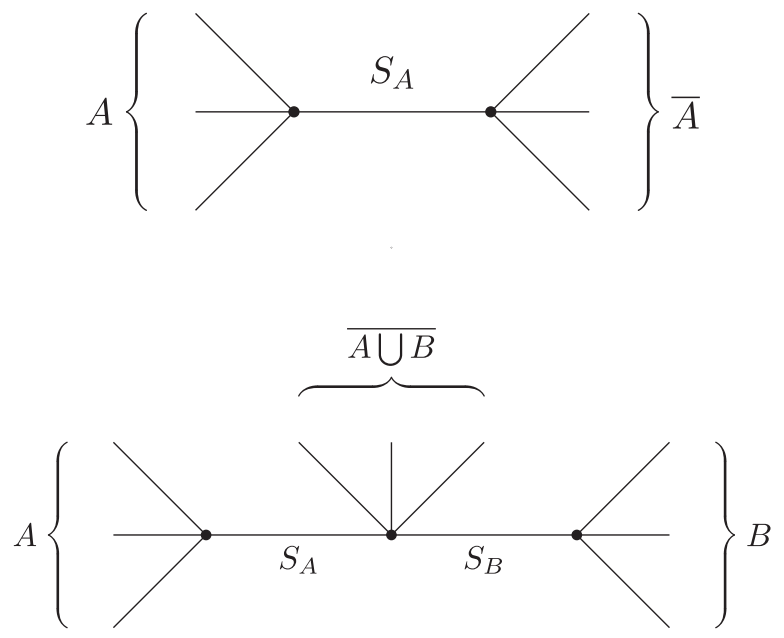

FIG. 4. The effective Feynman diagrams: the above is for only one singular poles, while the below is for two compatible singular poles.

In fact, we can get more accurate information, i.e., the speed of every point in $A$ going to the same location. Assuming $\left|z_{i}-z_{j}\right| \sim t^{N} \forall i, j \in A$, let us study the $\epsilon$ order of various part in the formula ${ }^{6}$ (1.4). Without loss of generality, we choose $S_{12 \ldots k} \rightarrow 0$ and fix $\{r, s, t\}=$ $1,2,3$ and $\{i, j, k\}=(n, n-1, n-2)$. And choose $S_{12 \ldots k} \rightarrow 0$. For the measure part

$$
J=\frac{\left(z_{r}-z_{s}\right)\left(z_{s}-z_{t}\right)\left(z_{t}-z_{r}\right)\left(z_{i}-z_{j}\right)\left(z_{j}-z_{k}\right)\left(z_{k}-z_{i}\right)}{|\Phi|_{i j k}^{r s t}}
$$

where matrix $\Phi$ is defined in (1.5), one can check that

$$
\begin{aligned}
\left(z_{1}-z_{2}\right)\left(z_{2}-z_{3}\right)\left(z_{3}-z_{1}\right)\left(z_{n}-z_{n-1}\right)\left(z_{n-1}-z_{n-2}\right)\left(z_{n-2}-z_{n}\right) & \sim \begin{cases}\epsilon^{N}, & |A|=2 \\
\epsilon^{3 N}, & |A| \geq 3\end{cases} \\
|\Phi|_{i j k}^{r s t} & \sim \begin{cases}\epsilon^{0}, & |A|=2 \\
\epsilon^{-2(|A|-3) N}, & |A| \geq 3\end{cases} \\
J & \sim \epsilon^{(2|A|-3) N}
\end{aligned}
$$

For the integrand part, the leading behavior is $\epsilon^{-N \complement[A]}$ where the $\mathbb{L}[A]$ is the linking number. Adding all together, we get the behavior $\epsilon^{-N(\mathbb{L}[A]-2(|A|-1)+1)}=\epsilon^{-N(\chi[A]+1)}$.

\footnotetext{
${ }^{5}$ Again, we use $A$ to denote the one with at most one gauge fixed point among the subsets $A, \bar{A}$.

${ }^{6}$ For the regular solutions, both measure and integrand will give $\epsilon^{0}$ contributions.
}

From the integration rule [31-33], the behavior is $S_{A}^{-(\chi[A]+1)} \sim \epsilon^{-(\chi[A]+1)}$, thus the match up of arbitrary choice of $\chi[A]$ requires $N=1$. One interesting point of the above analysis of contributions of singular solutions is that when $\chi[A]=-1$, we will have $\epsilon^{0}$. In other words, to get the right numerical result in the $\epsilon \rightarrow 0$ limit, we must include the contributions of singular solutions although analytic expression does not contain the pole $S_{A}$. 


\section{The numerical checking}

Now we present some numerical computations to verify the above analysis. In numerical computation, one key is the construction of the kinematics having the wanted limit behavior. The detailed discussion has been given in Appendix. For the current case, i.e., with one and only one $S_{A} \rightarrow 0$, a straightforward way is to use the Britto-Cachazo-Feng-Witten (BCFW)-deformation

[see (A8)-(A10)].

Example I: Six points with $S_{123} \rightarrow 0$

The kinematics is given by

$$
\begin{aligned}
k_{1} \rightarrow & \{-0.152-0.593 i,-1.019-0.981 i, 0.106+2.464 i,-0.560+1.268 i,-2.642+0.175 i\} \\
& +\{0.254,0.4205,-0.238-0.484 i,-0.1242-0.248 i, 0.461-0.317 i\} \epsilon \\
k_{2} \rightarrow & \{1.220,-0.852,-0.262,-0.825,0.115\} \\
k_{3} \rightarrow & \{1.586,-0.999,-0.459,-0.664,0.931\} \\
k_{4} \rightarrow & \{1.203,-0.240,0.419,0.968,0.527\} \\
k_{5} \rightarrow & \{-1.416,-0.0899,-0.750,-0.688,0.980\} \\
k_{6} \rightarrow & \{-2.441+0.5935 i,+3.201+0.981 i,+0.946-2.464 i,+1.770-1.267 i, 0.089-0.175 i\} \\
& -\{0.254,0.4205,-0.238-0.484 i,-0.1242-0.248 i, 0.461-0.317 i\} \epsilon
\end{aligned}
$$

We fix the gauge of $z_{4}=1, z_{5}=0, z_{6}=1$. There are 6 solutions with one singular and 5 regular solutions. The solutions are shown below as the series of $\epsilon$ up to $\epsilon^{1}$ terms:

$$
\begin{gathered}
\text { Singular }(1)\left\{\begin{array}{l}
z_{1} \rightarrow-(0.701112-0.144145 i)+(-3.14275-0.280886 i) \epsilon \\
z_{2} \rightarrow-(0.701112-0.144145 i)+(-3.20442-0.255032 i) \epsilon \\
z_{3} \rightarrow-(0.701112-0.144145 i)+(-3.18786-0.260227 i) \epsilon
\end{array}\right. \\
\text { Regular (1) }\left\{\begin{array}{l}
z_{1} \rightarrow(0.0452115+0.636781 i)-(0.0913283+0.0933938 i) \epsilon \\
z_{2} \rightarrow-(2.63638+0.252226 i)+(1.32192+0.0640334 i) \epsilon \\
z_{3} \rightarrow-(8.15521-3.66499 i)+(7.11037-9.61685 i) \epsilon
\end{array}\right. \\
\text { Regular (2) }\left\{\begin{array}{l}
z_{1} \rightarrow(0.0308196+0.774219 i)-(0.0791472+0.145376 i) \epsilon \\
z_{2} \rightarrow-(0.71447+6.1477 i)+(-6.30372+0.80879 i) \epsilon \\
z_{3} \rightarrow-(1.67537-0.0415368 i)+(0.253797-0.132973 i) \epsilon
\end{array}\right. \\
\text { Regular(3) }\left\{\begin{array}{l}
z_{1} \rightarrow-(0.28604-0.376317 i)+(-0.0471353-0.097799 i) \epsilon \\
z_{2} \rightarrow-(4.08226+0.917673 i)+(2.66599+1.09849 i) \epsilon \\
z_{3} \rightarrow-(0.377007-0.515852 i)+(-0.0729845-0.0909321 i) \epsilon
\end{array}\right. \\
\operatorname{Regular}(4)\left\{\begin{array}{l}
z_{1} \rightarrow-(0.435329-0.28238 i)+(0.0150451-0.0647125 i) \epsilon \\
z_{2} \rightarrow-(0.297746-0.197856 i)+(-0.0197837-0.00948061 i) \epsilon \\
z_{3} \rightarrow-(3.40278-1.35514 i)+(0.562885-0.896193 i) \epsilon
\end{array}\right. \\
\operatorname{Regular}(5)\left\{\begin{array}{l}
z_{1} \rightarrow-(0.72372-0.14116 i)+(0.0430294-0.0225452 i) \epsilon \\
z_{2} \rightarrow-(0.558638-0.130488 i)+(-0.00452728-0.0035687 i) \epsilon \\
z_{3} \rightarrow-(0.855428-0.12072 i)+(-0.0054946-0.00384179 i) \epsilon
\end{array}\right.
\end{gathered}
$$

The measure part of each solution is shown in Table I. which is indeed the behavior $\epsilon^{(2|A|-3)}=\epsilon^{2 \times 3-3}=\epsilon^{3}$. Now we choose three different CHY-integrands with pole index of $\chi\left[S_{123}\right]=0,-1,-2$ respectively to calculate the contribution of each solution. 
TABLE I. The measure part of each solution.

\begin{tabular}{lc}
\hline \hline Solution & Measure \\
\hline Singular (1) & $\left(1.08633 \times 10^{-7}-3.24352 \times 10^{-8} i\right) \epsilon^{3}$ \\
Regular (1) & $-(516.832+220.062 i)$ \\
Regular (2) & $-(6.23504+50.3245 i)$ \\
Regular (3) & $(0.0453058-0.141481 i)$ \\
Regular (4) & $(0.0188278+0.0202573 i)$ \\
Regular (5) & $-\left(3.4399 \times 10^{-6}+8.80267 \times 10^{-6} i\right)$ \\
\hline \hline
\end{tabular}

TABLE II. The contribution of each solution for the CHYIntegrand $P T(123456) P T(124563)$.

\begin{tabular}{lc}
\hline \hline Solution & Integration \\
\hline Singular (1) & $\frac{1}{\epsilon}(0.0801588+0.0445719 i)$ \\
Regular (1) & $-(0.00189857+0.00277649 i)$ \\
Regular (2) & $(0.00417627+0.00436192 i)$ \\
Regular (3) & $-(0.00185648+0.00211634 i)$ \\
Regular (4) & $-(0.00963083-0.000139444 i)$ \\
Regular (5) & $(0.0509707+0.00619341 i)$ \\
\hline \hline
\end{tabular}

(i) $I_{1}=P T(123456) P T(124563) \quad$ with $\quad \chi\left[S_{123}\right]=0$. The contribution of each solution is shown in Table II.

Summing up all six solutions, we have

$$
\frac{0.0801588+0.0445719 i}{\epsilon}+O\left(\epsilon^{0}\right)
$$

If we substitute the numerical kinematics (3.3) to the analytic expression

$$
\begin{aligned}
& \frac{1}{S_{12} S_{45} S_{123}}+\frac{1}{S_{12} S_{56} S_{123}} \\
& \quad=\frac{0.0802693+0.0448299 i}{\epsilon}+O\left(\epsilon^{0}\right)
\end{aligned}
$$

This result is essentially in agreement with (3.10). For this case, the leading contribution comes from the singular solution only.

(ii) $I_{2}=P T(123456) P T(124536)$ with $\chi\left[S_{123}\right]=-1$. The result is shown in Table III. The summation of six solutions is

$$
-(0.033591+0.0023464 i)+O \epsilon)
$$

which is identical with the numerical result by substituting (3.3) to analytic expression
TABLE III. The contribution of each solution of CHY-Integrand $P T(123456) P T(124536)$.

\begin{tabular}{lc}
\hline \hline Solution & Integration \\
\hline Singular (1) & $-(0.0188869-0.00321464 i)$ \\
Regular (1) & $(0.00236768+0.00127038 i)$ \\
Regular (2) & $-(0.00430291+0.00290199 i)$ \\
Regular (3) & $(0.000865392+0.000279345 i)$ \\
Regular (4) & $(0.011857-0.00567046 i)$ \\
Regular (5) & $-(0.0254912-0.00146168 i)$ \\
\hline \hline
\end{tabular}

TABLE IV. The contribution of each solution of CHY-Intergrand PT(123456)PT(143526).

\begin{tabular}{lc}
\hline \hline Solution & Integration \\
\hline Singular (1) & $(0.00100813-0.000287969 i) \epsilon$ \\
Regular (1) & $-(0.001916+0.00405212 i)$ \\
Regular (2) & $(0.000756028+0.000975639 i)$ \\
Regular (3) & $-(0.0000417965-0.000573095 i)$ \\
Regular (4) & $-(0.00286251-0.00330538 i)$ \\
Regular (5) & $-(0.0012266+0.00080199 i)$ \\
\hline \hline
\end{tabular}

$$
\begin{aligned}
& \frac{1}{S_{12} S_{45} S_{126}}+\frac{1}{S_{16} S_{45} S_{126}} \\
& \quad=-(0.033591+0.0023464 i)+O(\epsilon),
\end{aligned}
$$

This shows the correctness of (3.4) and (3.9). From Table III we see all solutions (including the singular solution) gives $\epsilon^{0}$ contribution.

(iii) $I_{3}=P T(123456) P T(143526)$ with $\chi\left[S_{123}\right]=-2$. The result is shown in Table IV. The summation of all six solutions is

$$
-0.00529087+O(\epsilon)
$$

which is identical to the analytic expression with the numerical kinematic (3.3)

$$
-\frac{1}{S_{16} S_{34} S_{126}}=-0.00529087+O(\epsilon)
$$

where singular solution will give no contribution when $\epsilon \rightarrow 0$.

Example II: six points with $S_{12} \rightarrow 0$. The choice of numerical kinematics is 


$$
\begin{aligned}
k_{1} \rightarrow & \{0.111+0.384 i,-1.887-0.493 i, 0.221-2.605 i,-0.751-1.544 i, 2.344-0.627 i\} \\
& +\{0.361,0.755+0.428 i,-0.363+0.731 i,-0.123+0.466 i,-0.589\} \epsilon \\
k_{2} \rightarrow & \{0.894,-0.453,-0.411,0.647,-0.0763\} \\
k_{3} \rightarrow & \{1.065,-0.183,0.780,0.557,0.427\} \\
k_{4} \rightarrow & \{1.387,-0.0137,-0.981,0.840,0.504\} \\
k_{5} \rightarrow & \{-1.7085,1.1005,-1.083,0.2038,-0.7025\} \\
k_{6} \rightarrow & \{-1.748-0.384 i, 1.436+0.493 i, 1.475+2.605 i,-1.497+1.544 i,-2.497+0.627 i\} \\
& -\{0.361,0.755+0.428 i,-0.363+0.731 i,-0.123+0.466 i,-0.589\} \epsilon .
\end{aligned}
$$

We fix the gauge choice $z_{4}=1, z_{5}=0, z_{6}=1$, there are two singular and four regular solutions:

$$
\begin{gathered}
\text { Singular }(1)\left\{\begin{array}{l}
z_{1} \rightarrow(0.762074+0.364077 i)-(0.0646452+0.838813 i) \epsilon \\
z_{2} \rightarrow(0.762074+0.364077 i)+(0.515056-0.754896 i) \epsilon \\
z_{3} \rightarrow-(0.0322539-0.0615108 i)+(0.01392+0.00452414 i) \epsilon
\end{array}\right. \\
\text { Singular }(2)\left\{\begin{array}{l}
z_{1} \rightarrow(0.112028-0.54179 i)-(0.047236-0.318322 i) \epsilon \\
z_{2} \rightarrow(0.112028-0.54179 i)+(0.225107+0.143289 i) \epsilon \\
z_{3} \rightarrow(0.396924+0.0453154 i)-(0.0887612-0.0250629 i) \epsilon
\end{array}\right. \\
\operatorname{Regular}(1)\left\{\begin{array}{l}
z_{1} \rightarrow(1.05565+0.418985 i)-(0.51277+0.296097 i) \epsilon \\
z_{2} \rightarrow(4.03218+1.32966 i)+(6.55886+28.4338 i) \epsilon \\
z_{3} \rightarrow-(0.0320861-0.0733717 i)+(-0.0125772+0.00408331 i) \epsilon
\end{array}\right. \\
\operatorname{Regular}(2)\left\{\begin{array}{l}
z_{1} \rightarrow(0.305812-0.703593 i)-(0.205823-0.327967 i) \epsilon \\
z_{2} \rightarrow-(4.30772+0.129581 i)+(15.332+1.98828 i) \epsilon \\
z_{3} \rightarrow(0.452278-0.0114679 i)-(0.109894-0.0917084 i) \epsilon
\end{array}\right. \\
\operatorname{Regular}(3)\left\{\begin{array}{l}
z_{1} \rightarrow(0.932462+0.508345 i)-(0.0889137+0.704417 i) \epsilon \\
z_{2} \rightarrow-(0.147739-0.337423 i)+(-0.0381437+0.0380429 i) \epsilon \\
z_{3} \rightarrow-(0.0558585-0.162427 i)+(-0.0398685-0.0252863 i) \epsilon
\end{array}\right. \\
\operatorname{Regular}(4)\left\{\begin{array}{l}
z_{1} \rightarrow(0.290776-0.394965 i)-(0.208807-0.274043 i) \epsilon \\
z_{2} \rightarrow(0.548109+0.0301496 i)-(0.132396-0.052737 i) \epsilon \\
z_{3} \rightarrow(0.296957+0.0219136 i)-(0.142326-0.0932441 i) \epsilon
\end{array}\right.
\end{gathered}
$$

The measure part of each solution is shown in Table V. which agrees with our analysis $\epsilon^{0}$ for regular solutions and $\epsilon^{2|A|-3}$ for singular solutions. Again we choose three different CHY-integrands with pole index $\chi\left[S_{12}\right]=0,-1,-2$ respectively.

(i) $I_{1}=P T(123456) P T(124563)$ with $\chi\left[S_{12}\right]=0$. The result is shown in VI. The summation of six solutions is

$$
-\frac{0.0109398-0.0160091 i}{\epsilon}+O(\epsilon)
$$

which is identical with the analytic expression with the numerical kinematic (3.16)

$$
\frac{1}{S_{12} S_{45} S_{123}}+\frac{1}{S_{12} S_{56} S_{123}}=-\frac{0.0109398-0.0160091 i}{\epsilon}+O(\epsilon)
$$

where the leading contribution comes from the singular solutions only.

(ii) $I_{2}=P T(123456) P T(134526)$ with $\chi\left[S_{12}\right]=-1$. The result is shown in Table VII. The fact that summation of six solutions 
TABLE V. The measure part of each solution for $S_{12} \rightarrow 0$.

\begin{tabular}{lc}
\hline \hline Solution & Measure \\
\hline Singular (1) & $-(0.000529048+0.0005798 i) \epsilon$ \\
Singular (2) & $(0.000892278+0.000357788 i) \epsilon$ \\
Regular (1) & $-(0.186684+0.928303 i)$ \\
Regular (2) & $-(1.95152-1.51335 i)$ \\
Regular (3) & $-(0.0000405857-0.000616897 i)$ \\
Regular (4) & $-(0.000169266-0.0000142748 i)$ \\
\hline \hline
\end{tabular}

TABLE VI. The contribution of each solution of CHY-integrand $P T(123456) P T(124563)$.

\begin{tabular}{lc}
\hline \hline Solution & Integration \\
\hline Singular (1) & $\frac{1}{\epsilon}(0.00333026+0.00227831 i)$ \\
Singular (2) & $-\frac{1}{\epsilon}(0.0142701-0.0137308 i)$ \\
Regular (1) & $-(0.00260062-0.0012496 i)$ \\
Regular (2) & $(0.00122598-0.00527255 i)$ \\
Regular (3) & $-(0.000501431-0.000889243 i)$ \\
Regular (4) & $(0.0115214-0.00241242 i)$ \\
\hline \hline
\end{tabular}

TABLE VII. The contribution of each solutions of CHYintegrand $P T(123456) P T(134526)$.

\begin{tabular}{lc}
\hline \hline Solution & Integration \\
\hline Singular (1) & $-(0.000196838-0.000292468 i)$ \\
Singular (2) & $-(0.000377502-0.0182017 i)$ \\
Regular (1) & $-(0.000561198-0.000217058 i)$ \\
Regular (2) & $(0.00839347-0.0144827 i)$ \\
Regular (3) & $(0.00167404-0.00059116 i)$ \\
Regular (4) & $-(0.00221397+0.00363733 i)$ \\
\hline \hline
\end{tabular}

$$
0.006718+O(\epsilon)
$$

is identical with analytic result

$$
\frac{1}{S_{16} S_{34} S_{126}}+\frac{1}{S_{16} S_{45} S_{126}}=0.006718+O(\epsilon)
$$

shows that when $\epsilon \rightarrow 0$, both regular and singular solutions give nonzero contributions.

(iii) $I_{3}=P T(132456) P T(134526)$ with pole index $\chi\left[S_{12}\right]=-2$. The result is shown in VIII. The summation of six solutions is

$$
-(0.00372607+0.00225224 i)+O(\epsilon)
$$

which agrees with the analytic result

$$
\begin{aligned}
& \frac{1}{S_{13} S_{45} S_{136}}+\frac{1}{S_{16} S_{45} S_{136}} \\
& \quad=-(0.00372607+0.00225224 i)+O(\epsilon) .
\end{aligned}
$$

TABLE VIII. The contribution of each solutions of CHYintegrand $P T(132456) P T(134526)$.

\begin{tabular}{lc}
\hline \hline Solution & Integration \\
\hline Singular (1) & $-(0.000557274-0.000151919 i) \epsilon$ \\
Singular (2) & $-(0.00394239-0.00346853 i) \epsilon$ \\
Regular (1) & $(0.00033303-0.00039 i)$ \\
Regular (2) & $-(0.00932611+0.00647014 i)$ \\
Regular (3) & $-(0.00153402-0.00062326 i)$ \\
Regular (4) & $(0.00680103+0.00398464 i)$ \\
\hline \hline
\end{tabular}

For this case, when $\epsilon \rightarrow 0$, the regular solutions give whole contribution and singular solutions give no contributions.

\section{B. With $S_{A}, S_{B} \rightarrow 0$}

Now we consider the factorization limit where two poles $S_{A}, S_{B}$ go to zero at the same time. As discussed in the Appendix, a way to reach the kinematic configuration is to use two pairs of BCFW-deformations, for example,

$$
\begin{aligned}
p_{1}\left(w_{1}\right) & =p_{1}+w_{1} q_{1 n}, \quad p_{n}\left(w_{1}\right)=p_{n}-w_{1} q_{1 n}, \\
q_{1 n}^{2} & =q_{1 n} \cdot p_{1}=q_{1 n} \cdot p_{n}=0 \\
p_{2}\left(w_{2}\right) & =p_{2}+w_{2} q_{2 m}, \quad p_{m}\left(w_{2}\right)=p_{m}-w_{2} q_{2 m}, \\
q_{2 m}^{2} & =q_{2 m} \cdot p_{1}=q_{2 m} \cdot p_{m}=0 .
\end{aligned}
$$

Using $S_{A}=S_{B}=0$ we can solve $w_{1}^{*}, w_{2}^{*}$, then we write $w_{1}=w_{1}^{*}+\epsilon$ and $w_{2}=w_{2}^{*}+\epsilon$ and $\epsilon \rightarrow 0$ will give $S_{A}, S_{B} \rightarrow 0$ at the same time.

A new feature of two poles going to zero at the same time is that there are two different cases we should consider. The first case is that two poles are compatible, i.e., two poles appear in a single Feynman diagram. The second case is that two poles are not compatible, so there is no Feynman diagram containing them simultaneously. While the second case is that they are not compatible, let us discuss them one by one.

\section{1. $S_{A}, S_{B}$ are not compatible}

For this case, we will have $A \not \subset B$ and $A \not \subset \bar{B}$, so at least one intersection $A \cap B$ or $A \cap \bar{B}$ will be the true nonempty subset. Therefore, the first claim we can give is that there are singular solutions for $S_{A}$ and $S_{B}$. The second question is that are there "common singular solutions"? If there is a common singular solution, $z_{i} \rightarrow z_{A}, \quad \forall i \in A$ and $z_{j} \rightarrow z_{B}, \forall j \in B$ will lead $z_{A}=z_{B}$, thus by the analysis in Sec. II. We will get $S_{A \cup B} \rightarrow 0$, which is a conflict with our assumption of kinematic configuration. Therefore, we conclude that there is no common singular solution when $S_{A}, S_{B}$ are not compatible. This result should be easy to guess since there is no Feynman diagram containing them simultaneously.

Third, we discuss the collapse speed of singular solutions, i.e., $\left|z_{i}-z_{j}\right| \sim \epsilon^{N}, \quad \forall i, j \in A$. The analysis will be 
the exact same as in a previous subsection and we conclude that $N=1$, thus contributions of singular solutions of $a_{A}$ will be the order $\epsilon^{-1+\chi(A)}$ (similar for $S_{B}$ ). Finally the number of singular solutions for $S_{A}$ and $S_{B}$ will be $\left(n_{A}-\right.$ $2) !\left(n-n_{A}-2\right)$ ! and $\left(n_{B}-2\right) !\left(n-n_{B}-2\right)$ ! respectively.

\section{2. $S_{A}, S_{B}$ are compatible}

For this case, we can make $A \cap B=\varnothing$. Again the first result is that there are singular solutions for $S_{A}$ and $S_{B}$. To see if there is any common singular solution, we can consider the CHY-integrands, which give Feynman diagrams containing both poles $S_{A}, S_{B}$. If there is no common singular solution, by the same analysis [see (5.4)], we can see that the measure gives either $\epsilon^{(2|A|-3) N_{A}}$ or $\epsilon^{(2|A|-3) N_{B}}$, thus the leading behavior will be either $\epsilon^{-N_{A}(\chi[A]+1)}$ or $\epsilon^{-N_{B}(\chi[A]+1)}$, but from Feynman diagrams, we see the leading behavior $\epsilon^{-(\chi[A]+1)} \epsilon^{-(\chi[B]+1)}$. We cannot get the match result since $N_{A}, N_{B}$ are fixed while choosing different $\chi[A], \chi[B]$. Thus the contradiction tells us that there are common singular solutions.

One intuitive way to see the number of common singular solution is to draw the effective Feynman diagram (see
Fig. 4). For only one $S_{A} \rightarrow 0$, the left effective Feynman diagram gives the right accounting. For two compatible poles, the effective Feynman diagrams are given at the below of Fig. 4. Thus all legs have been divided into three parts: the left part contains $n_{A}+1$ external legs, the middle part contains $n_{A \bar{\cup} B}+2$ external legs, and the right part includes $n_{B}+1$ external legs. With the picture, the whole $n$-point will contains $\left(n_{A}+1-3\right) ! \times\left(n_{\overline{A \cup B}}+2-3\right) ! \times$ $\left(n_{B}+1-3\right) !=\left(n_{A}-2\right) !\left(n_{\overline{A \cup B}}-1\right) !\left(n_{B}-2\right)$ ! common singular solutions.

Now we discuss the collapse speed of singular solutions, i.e., $\left|z_{i}-z_{j}\right| \sim \epsilon^{N}, \forall i, j \in A(B)$. For the three types of singular solutions, by matching up the singular behavior of Feynman diagrams, one can see that for the singular solution of $S_{A}$ only, we will have $\left|z_{i}-z_{j}\right| \sim \epsilon, \forall i, j \in$ $A$ and $\left|z_{i}-z_{j}\right| \sim \epsilon^{0}, \forall i, j \in B$. ${ }^{7}$ Similar result holds for singular solution of $S_{B}$ only, i.e., $\left|z_{i}-z_{j}\right| \sim \epsilon, \quad \forall i, j \in B$ and $\left|z_{i}-z_{j}\right| \sim \epsilon^{0}, \forall i, j \in A$. For the common singular solutions, the analysis is tricky. Because the asymptotic behavior depends on the choice of gauge fixing $z_{i}, z_{j}, z_{k}$, we can have two types of asymptotic behavior. The first one is

$$
\begin{aligned}
& \text { type - I: } z_{i \in A} \rightarrow z_{A}+a_{i} \epsilon^{N_{1}}, \quad\left|a_{i}-a_{j}\right| \neq 0, \quad i, j \in A \\
& z_{t \in B} \rightarrow z_{B}+a_{t} \epsilon^{N_{2}}, \quad\left|a_{t}-a_{s}\right| \neq 0, \quad t, s \in B, \quad z_{A} \neq z_{B}
\end{aligned}
$$

The second type is

$$
\begin{aligned}
\text { type }-\mathrm{II}: z_{i \in A} & \rightarrow z_{B}+z_{A} \epsilon^{N_{1}}+a_{i} \epsilon^{N_{2}}, \quad\left|a_{i}-a_{j}\right| \neq 0, \quad i, j \in A, \quad N_{2}>N_{1} \\
z_{t \in B \backslash A} & \rightarrow z_{B}+a_{t} \epsilon^{N_{1}}, \quad\left|a_{t}-z_{A}\right| \neq 0, \quad\left|a_{t}-a_{s}\right| \neq 0, \quad t, s \in B \backslash A
\end{aligned}
$$

The type-I can be realized that if we take one gauge fixed point in $A$, one gauge fixed point in $B$ and the third point in $\overline{A \cup B}$ with the choice $A \cap B=\varnothing$. The type-II can be realized by taking one gauge fixed point in $B \backslash A$ and two gauge-fixed points in $\bar{B}$ with the choice $A \subset B$. Suppose the gauge choice makes the type-I behavior by analyzing the leading behavior of measure and integrand in the previous subsection. In that case, one can check that we should have $N_{1}=N_{2}=1$ to match up the result from Feynman diagrams. If the gauge choice makes the type-II behavior, a similar argument leads $N_{1}=1, N_{2}=2$.

Knowing the value of $N_{1}, N_{2}$, we see the measure part of the common singular solutions gives $\epsilon^{2|A|-3} \cdot \epsilon^{2|B|-3}$, while the leading contribution gives $\epsilon^{-(\chi[A]+1)} \cdot \epsilon^{-(\chi[B]+1)}$. $^{8}$

\section{Numerical check}

In this part, we will present several numerical examples to demonstrate above analysis.

Example I: $n=7, S_{12}=S_{45}=0$

We first consider the case of $S_{A}$ is compatible with $S_{B}$. We will choice $n=7, A=\{1,2\}, B=\{4,5\}$. The kinematics configuration is

\footnotetext{
${ }^{7}$ Noticing that we have assumed $A \cap B=\varnothing$. Otherwise, we use $\bar{B}$ to replace $B$.

${ }^{8}$ Although the asymptotic behavior of type- $I$ and type- $I I$ are different, their leading behavior of measure and contribution have the same $\epsilon$-order. Which can be calculated by the same procedure of the previous subsection staring by staring with (3.26) and (3.27) respectively.
} 


$$
\begin{aligned}
k_{1} \rightarrow & \{0.502+0.165 i,-0.303+0.197 i,-0.326-0.315 i,-0.109-0.0949 i,-0.409-0.0717 i\} \\
& +\{0.616 \epsilon,(0.336-0.195 i) \epsilon,(-0.119+0.516 i) \epsilon,(-0.719-0.177 i) \epsilon,-0.269 \epsilon\} \\
k_{2} \rightarrow & \{1.2,-0.256,-0.731,0.424,-0.818\} \\
k_{3} \rightarrow & \{1.19-0.165 i, 0.728-0.197 i, 0.11+0.315 i,-0.99+0.0949 i, 0.102+0.0717 i\} \\
& +\{-0.616 \epsilon,(-0.336+0.195 i) \epsilon,(0.119-0.516 i) \epsilon,(0.719+0.177 i) \epsilon, 0.269 \epsilon\} \\
k_{4} \rightarrow & \{0.361+0.689 i,-0.646-0.902 i, 0.705-0.421 i, 0.0459-0.54 i,-0.162+0.0756 i\} \\
& +\{0.96 \epsilon,(-0.779+0.414 i) \epsilon,(-0.99-0.349 i) \epsilon,(-0.0376+0.619 i) \epsilon, 0.105 \epsilon\} \\
k_{5} \rightarrow & \{1 .,-0.394,0.288,-0.796,0.366\} \\
k_{6} \rightarrow & \{-1.76,0.214,-1.52,0.246,-0.82\} \\
k_{7} \rightarrow & \{-2.49-0.689 i, 0.657+0.902 i, 1.48+0.421 i, 1.18+0.54 i, 1.74-0.0756 i\} \\
& +\{-0.96 \epsilon,(0.779-0.414 i) \epsilon,(0.99+0.349 i) \epsilon,(0.0376-0.619 i) \epsilon,-0.105 \epsilon\} .
\end{aligned}
$$

We fix $z_{3}=1, z_{6}=5, z_{7}=-7$, thus the type-I behavior is realized. There are 10 singular solutions in total:

$$
\begin{aligned}
& \text { Singular } S_{12} S_{45}(1)\left\{\begin{array}{l}
z_{1} \rightarrow(-0.19+7.49 i)-(19.4-1.93 i) \epsilon-(21.3+32.7 i) \epsilon^{2} \\
z_{2} \rightarrow(-0.19+7.49 i)-(4.74+3.58 i) \epsilon+(3.28-6.28 i) \epsilon^{2} \\
z_{4} \rightarrow(11.4+0.699 i)+(7.43-8.4 i) \epsilon+(2.68-4.86 i) \epsilon^{2} \\
z_{5} \rightarrow(11.4+0.699 i)+(3.62+1.88 i) \epsilon-(4.62-7.69 i) \epsilon^{2}
\end{array}\right. \\
& \text { Singular } S_{12} S_{45}(2)\left\{\begin{aligned}
z_{1} & \rightarrow(-8.16-2.93 i)+(4.47+4.09 i) \epsilon-(2.34+4.47 i) \epsilon^{2} \\
z_{2} & \rightarrow(-8.16-2.93 i)+(2.59-1.07 i) \epsilon+(2.6-5.33 i) \epsilon^{2} \\
z_{4} & \rightarrow(2.41+2.42 i)+(1.69+6.53 i) \epsilon-(1.56+0.251 i) \epsilon^{2} \\
z_{5} & \rightarrow(2.41+2.42 i)+(0.484+0.193 i) \epsilon-(1.83-1.95 i) \epsilon^{2}
\end{aligned}\right. \\
& \text { Singular } S_{12}(1)\left\{\begin{aligned}
z_{1} & \rightarrow(6.09+26.5 i)-(147 .-111 . i) \epsilon-(850 .+129 . i) \epsilon^{2} \\
z_{2} & \rightarrow(6.09+26.5 i)-(36.3-28.2 i) \epsilon-(129 .-2.6 i) \epsilon^{2} \\
z_{4} & \rightarrow(23.2-27 . i)-(42.3+79.6 i) \epsilon+(173 .-208 . i) \epsilon^{2} \\
z_{5} & \rightarrow(2.47+1.81 i)-(1.14-0.729 i) \epsilon-(5.27+1.87 i) \epsilon^{2}
\end{aligned}\right. \\
& \text { Singular } S_{12}(2)\left\{\begin{aligned}
z_{1} & \rightarrow(-0.326+7.62 i)-(21.1-5.41 i) \epsilon-(45.6+41.3 i) \epsilon^{2} \\
z_{2} & \rightarrow(-0.326+7.62 i)-(4.44+0.898 i) \epsilon+(1 .-3.95 i) \epsilon^{2} \\
z_{4} & \rightarrow(6.96+16.3 i)-(11.6-14 . i) \epsilon-(17.4+30.4 i) \epsilon^{2} \\
z_{5} & \rightarrow(4.61-4.29 i)-(2.76+1.31 i) \epsilon+(1.47-1.54 i) \epsilon^{2}
\end{aligned}\right. \\
& \text { Singular } S_{12}(3)\left\{\begin{aligned}
z_{1} & \rightarrow(-8.34+0.879 i)-(3.38+4.78 i) \epsilon+(7.54+1.7 i) \epsilon^{2} \\
z_{2} & \rightarrow(-8.34+0.879 i)-(5.22+2.61 i) \epsilon-(2.47+2.18 i) \epsilon^{2} \\
z_{4} & \rightarrow(1.54+1.85 i)-(0.644-3.99 i) \epsilon-(4.87+1.35 i) \epsilon^{2} \\
z_{5} & \rightarrow(-4.29-5.26 i)+(18.2+3.21 i) \epsilon-(5.86-9.66 i) \epsilon^{2}
\end{aligned}\right. \\
& \text { Singular } S_{12}(4)\left\{\begin{array}{l}
z_{1} \rightarrow(-7.12-3.13 i)+(6.76+4 . i) \epsilon-(10.1+10.1 i) \epsilon^{2} \\
z_{2} \rightarrow(-7.12-3.13 i)+(6.87-1.01 i) \epsilon-(0.647+10.5 i) \epsilon^{2} \\
z_{4} \rightarrow(1.67+1.96 i)-(0.965-3.22 i) \epsilon-(2.25-6.98 i) \epsilon^{2} \\
z_{5} \rightarrow(2.82+6.01 i)+(5.02+19.3 i) \epsilon+(30.2-13.2 i) \epsilon^{2}
\end{array}\right.
\end{aligned}
$$




$$
\begin{aligned}
& \text { Singular } S_{45}(1)\left\{\begin{array}{l}
z_{1} \rightarrow(-2.33+29.4 i)-(85.6+2.5 i) \epsilon+(24.1-22.3 i) \epsilon^{2} \\
z_{2} \rightarrow(0.596+6.55 i)-(6.3+2.18 i) \epsilon-(1.86+8.77 i) \epsilon^{2} \\
z_{4} \rightarrow(14.4+2.23 i)+(15.6-21.3 i) \epsilon+(21.6-13.2 i) \epsilon^{2} \\
z_{5} \rightarrow(14.4+2.23 i)+(6.56-4.88 i) \epsilon+(11.8+0.324 i) \epsilon^{2}
\end{array}\right. \\
& \text { Singular } S_{45}(2)\left\{\begin{array}{l}
z_{1} \rightarrow(-0.959+1.13 i)-(1.06+0.33 i) \epsilon-(1.02+0.891 i) \epsilon^{2} \\
z_{2} \rightarrow(4.6+6.79 i)-(7.89-6.2 i) \epsilon-(9.72+0.0344 i) \epsilon^{2} \\
z_{4} \rightarrow(8.42-0.152 i)+(5.02-4.83 i) \epsilon+(3.21-5.33 i) \epsilon^{2} \\
z_{5} \rightarrow(8.42-0.152 i)+(4.05-0.0335 i) \epsilon+(3.11+3.2 i) \epsilon^{2}
\end{array}\right. \\
& \text { Singular } S_{45}(3)\left\{\begin{array}{l}
z_{1} \rightarrow(-2.69+1.77 i)-(2.51+0.646 i) \epsilon-(1.06+0.197 i) \epsilon^{2} \\
z_{2} \rightarrow(-5.6-7.92 i)-(2.68-2.24 i) \epsilon+(1.2+5.65 i) \epsilon^{2} \\
z_{4} \rightarrow(2.4+2.42 i)+(1.74+6.79 i) \epsilon-(0.271-3.09 i) \epsilon^{2} \\
z_{5} \rightarrow(2.4+2.42 i)+(0.631+0.616 i) \epsilon-(2.43-3.81 i) \epsilon^{2}
\end{array}\right. \\
& \text { Singular } S_{45}(4)\left\{\begin{array}{l}
z_{1} \rightarrow(1.06+2.21 i)-(3.67-1.25 i) \epsilon-(5.58+1.63 i) \epsilon^{2} \\
z_{2} \rightarrow(-4.1-6.76 i)-(0.276-0.326 i) \epsilon-(1.36-0.429 i) \epsilon^{2} \\
z_{4} \rightarrow(3.47+3.83 i)+(3.84+5.81 i) \epsilon+(0.612+0.62 i) \epsilon^{2} \\
z_{5} \rightarrow(3.47+3.83 i)+(0.535+0.828 i) \epsilon-(2.6-0.521 i) \epsilon^{2}
\end{array}\right.
\end{aligned}
$$

Among them there are two common singular solutions, six solutions for $S_{12}$, six solutions for $S_{45}$. The quantity and the asymptotic behaviors confirm our claim. The measure part for common singular solutions are given by Table IX, where for common singular solutions it is $\epsilon^{2|A|+2|B|-6}=\epsilon^{2}$, and for singular solutions of only one pole it is $\epsilon^{2|A|-3}=$ $\epsilon^{2|B|-3}=\epsilon^{1}$.

We choose a few CHY-integrands to see the effects of singular solutions. For the first integrand

$$
I_{1}=\frac{1}{z_{12}^{2} z_{17}^{2} z_{23} z_{24} z_{34} z_{35} z_{37} z_{45} z_{46} z_{56}^{2} z_{67}}
$$

with $\chi(12)=0$ and $\chi(45)=-1$. The contribution of each singular solution is in Table $\mathrm{X}$. The summation is

$$
\frac{0.0839+0.0704 i}{\epsilon}+O\left(\epsilon^{0}\right)
$$

while the corresponding amplitude result by substitute numerical kinematics is

$$
\frac{1}{S_{12} S_{56} S_{127} S_{456}}+\frac{1}{S_{17} S_{56} S_{127} S_{456}}=\frac{0.0839+0.0704 i}{\epsilon}+(0.0809+0.209 i)-(0.128-0.302 i) \epsilon+O\left(\epsilon^{2}\right) .
$$

The second integrand as

$$
I_{2}=-\frac{1}{z_{12}^{2} z_{13} z_{17} z_{23} z_{26} z_{34} z_{35} z_{45}^{2} z_{47} z_{56} z_{67}^{2}}
$$

with $\chi(12)=\chi(45)=0$. The contribution of each singular solution is in Table XI. The summation is

$$
-\frac{0.000722-0.00721 i}{\epsilon^{2}}+O\left(\frac{1}{\epsilon}\right)
$$

while the amplitude result by substitute numerical kinematics is

$$
-\frac{1}{S_{12} S_{45} S_{67} S_{123}}-\frac{1}{S_{12} S_{45} S_{67} S_{345}}=-\frac{0.000722-0.00721 i}{\epsilon^{2}}+\frac{0.00438-0.00799 i}{\epsilon}-(0.0122-0.00712 i)+O(\epsilon)
$$


TABLE IX. Measure part of each singular solution of $S_{12}=S_{45}=0$.

\begin{tabular}{lc}
\hline \hline Solution & Measure \\
\hline Singular $S_{12} S_{45}(1)$ & $\left(3.7 \times 10^{11}+\left(6.26 \times 10^{11}\right) i\right) \epsilon^{2}+O\left(\epsilon^{3}\right)$ \\
Singular $S_{12} S_{45}(2)$ & $-\left(1.83 \times 10^{8}+\left(1.23 \times 10^{9}\right) i\right) \epsilon^{2}+O\left(\epsilon^{3}\right)$ \\
Singular $S_{12}(1)$ & $\left(5.41 \times 10^{15}+\left(1.2 \times 10^{16}\right) i\right) \epsilon+O\left(\epsilon^{2}\right)$ \\
Singular $S_{12}(2)$ & $\left(3.74 \times 10^{11}+\left(1.57 \times 10^{12}\right) i\right) \epsilon+O\left(\epsilon^{2}\right)$ \\
Singular $S_{12}(3)$ & $-\left(1.22 \times 10^{8}-\left(6.38 \times 10^{7}\right) i\right) \epsilon+O\left(\epsilon^{2}\right)$ \\
Singular $S_{12}(4)$ & $\left(1.53 \times 10^{9}-\left(1.66 \times 10^{8}\right) i\right) \epsilon+O\left(\epsilon^{2}\right)$ \\
Singular $S_{45}(1)$ & $-\left(9.4 \times 10^{13}-\left(5.12 \times 10^{12}\right) i\right) \epsilon+O\left(\epsilon^{2}\right)$ \\
Singular $S_{45}(2)$ & $-\left(3.92 \times 10^{7}+\left(1.17 \times 10^{9}\right) i\right) \epsilon+O\left(\epsilon^{2}\right)$ \\
Singular $S_{45}(3)$ & $-\left(9.05 \times 10^{8}+\left(2.25 \times 10^{9}\right) i\right) \epsilon+O\left(\epsilon^{2}\right)$ \\
Singular $S_{45}(4)$ & $\left(6.1 \times 10^{8}-\left(4.74 \times 10^{8}\right) i\right) \epsilon+O\left(\epsilon^{2}\right)$ \\
\hline \hline
\end{tabular}

From the result of these two integrands, we can verify the behavior of the singular solutions. The contribution of the common singular solution is exact $\epsilon^{-2-\chi(12)-\chi(45)}, \epsilon^{-1-\chi(12)}$ for the singular solutions of $S_{12}$ and $\epsilon^{-1-\chi(45)}$ for the singular solutions of $S_{45}$, which agree with our analysis.
Example II: $n=7, S_{123}=S_{12}=0$

Next we consider another compatible case $A \subset B$ with $A=$ $\{12\}, B=\{123\}$. Our gauge choice is that $z_{i}, z_{j}, z_{k} \notin B$, thus the type-II behavior is realized. The kinematics of 7-point construction numerically we choose as

TABLE X. Contribution of each singular solution of $I_{1}$.

\begin{tabular}{lc}
\hline \hline Solution & Contribution \\
\hline Singular $S_{12} S_{45}(1)$ & $-\frac{0.00877-0.00326 i}{\epsilon}+O\left(\epsilon^{0}\right)$ \\
Singular $S_{12} S_{45}(2)$ & $\frac{0.107+0.13 i}{\epsilon}+O\left(\epsilon^{0}\right)$ \\
Singular $S_{12}(1)$ & $\frac{0.00589+0.000659 i}{\epsilon}+O\left(\epsilon^{0}\right)$ \\
Singular $S_{12}(2)$ & $\frac{0.00559+0.00778 i}{\epsilon}+O\left(\epsilon^{0}\right)$ \\
Singular $S_{12}(3)$ & $-\frac{0.00599-0.0111 i}{\epsilon}+O\left(\epsilon^{0}\right)$ \\
Singular $S_{12}(4)$ & $-\frac{0.0197+0.0828 i}{\epsilon}+O\left(\epsilon^{0}\right)$ \\
Singular $S_{45}(5)$ & $(0.00198-0.00667 i)+O\left(\epsilon^{1}\right)$ \\
Singular $S_{45}(6)$ & $(0.00348+0.00699 i)+O\left(\epsilon^{1}\right)$ \\
Singular $S_{45}(7)$ & $(0.0172+0.0341 i)+O\left(\epsilon^{1}\right)$ \\
Singular $S_{45}(8)$ & $(0.000144-0.00111 i)+O\left(\epsilon^{1}\right)$ \\
All Regulars & $-(0.0285+0.0362 i)-(0.0596+0.314 i) \epsilon+O\left(\epsilon^{2}\right)$ \\
\hline \hline
\end{tabular}

TABLE XI. Contribution of each singular solution of $I_{2}$.

\begin{tabular}{lc}
\hline \hline Solution & Contribution \\
\hline Singular $S_{12} S_{45}(1)$ & $\frac{0.0021+0.00137 i}{\epsilon^{2}}+O\left(\frac{1}{\epsilon}\right)$ \\
Singular $S_{12} S_{45}(2)$ & $-\frac{0.00282-0.00584 i}{\epsilon^{2}}+O\left(\frac{1}{\epsilon}\right)$ \\
Singular $S_{12}(1)$ & $\frac{0.000623+0.000173 i}{\epsilon}+O\left(\epsilon^{0}\right)$ \\
Singular $S_{12}(2)$ & $-\frac{0.0013-0.000968 i}{\epsilon}+O\left(\epsilon^{0}\right)$ \\
Singular $S_{12}(3)$ & $-\frac{0.000436+0.000344 i}{\epsilon}+O\left(\epsilon^{0}\right)$ \\
Singular $S_{12}(4)$ & $-\frac{0.00963-0.00522 i}{\epsilon}+O\left(\epsilon^{0}\right)$ \\
Singular $S_{45}(1)$ & $-\frac{0.0017+0.00104 i}{\epsilon}+O\left(\epsilon^{0}\right)$ \\
Singular $S_{45}(2)$ & $-\frac{0.00234+0.00102 i}{\epsilon}+O\left(\epsilon^{0}\right)$ \\
Singular $S_{45}(3)$ & $\frac{0.00355-0.00477 i}{\epsilon}+O\left(\epsilon^{0}\right)$ \\
Singular $S_{45}(4)$ & $-\frac{0.000814+0.000133 i}{\epsilon}+O\left(\epsilon^{0}\right)$ \\
All Regulars & $(0.0112-0.00641 i)+(0.00411+0.0247 i) \epsilon+O\left(\epsilon^{2}\right)$ \\
\hline \hline
\end{tabular}




$$
\begin{aligned}
k_{1} \rightarrow & \{5.78+1.74 i, 21.2-7.05 i, 0.742+0.329 i,-3.03+14.8 i,-11.8-17.3 i\} \\
& +\{0.638 \epsilon,(2.15-1.77 i) \epsilon, 0.12 \epsilon,(0.2+1.95 i) \epsilon,(-2.23-1.53 i) \epsilon\} \\
k_{2} \rightarrow & \{1.27+0.0208 i,-0.315-1.17 i, 0.27+0.0766 i,-0.115+0.124 i,-1.69+0.206 i\} \\
& +\{0.106 \epsilon,(0.0134+1.15 i) \epsilon, 0.389 \epsilon,(0.817+0.036 i) \epsilon,(0.726-0.0618 i) \epsilon\} \\
k_{3} \rightarrow & \{0.875,0.379,-0.717,0.329,-0.00131\} \\
k_{4} \rightarrow & \{1.7,-0.972,0.513,-0.912,0.918\} \\
k_{5} \rightarrow & \{1.05-0.0208 i, 0.283+1.17 i,-0.234-0.0766 i, 0.655-0.124 i, 1.41-0.206 i\} \\
& +\{-0.106 \epsilon,(-0.0134-1.15 i) \epsilon,-0.389 \epsilon,(-0.817-0.036 i) \epsilon,(-0.726+0.0618 i) \epsilon\} \\
k_{6} \rightarrow & \{-7.17-1.74 i,-20.1+7.05 i, 0.435-0.329 i, 5.4-14.8 i, 13.6+17.3 i\} \\
& +\{-0.638 \epsilon,(-2.15+1.77 i) \epsilon,-0.12 \epsilon,(-0.2-1.95 i) \epsilon,(2.23+1.53 i) \epsilon\} \\
k_{7} \rightarrow & \{-3.51,-0.482,-1.01,-2.33,-2.37\}
\end{aligned}
$$

We fix $z_{5}=1, z_{6}=5, z_{7}=-7$, the singular solutions are

$$
\begin{aligned}
& \text { Singular } S_{123} S_{12}(1)\left\{\begin{aligned}
z_{1} & \rightarrow(4.96-0.203 i)+(0.0866+0.743 i) \epsilon+(17.9+39.6 i) \epsilon^{2} \\
z_{2} & \rightarrow(4.96-0.203 i)+(0.0866+0.743 i) \epsilon-(94.6-51.5 i) \epsilon^{2} \\
z_{3} & \rightarrow(4.96-0.203 i)+(0.502-14 . i) \epsilon+(224 .-904 . i) \epsilon^{2} \\
z_{4} & \rightarrow(3.51+1.78 i)+(3.4+1.59 i) \epsilon-(2.95-7.46 i) \epsilon^{2}
\end{aligned}\right. \\
& \text { Singular } S_{123} S_{12}(2)\left\{\begin{array}{l}
z_{1} \rightarrow(4.89-0.552 i)+(0.158+2.21 i) \epsilon+(49 .+119 . i) \epsilon^{2} \\
z_{2} \rightarrow(4.89-0.552 i)+(0.158+2.21 i) \epsilon-(254 .-148 . i) \epsilon^{2} \\
z_{3} \rightarrow(4.89-0.552 i)+(1.68-37.7 i) \epsilon+(539 .-2460 . i) \epsilon^{2} \\
z_{4} \rightarrow(1.88+3.74 i)+(3.94+3.07 i) \epsilon+(59.4-0.813 i) \epsilon^{2}
\end{array}\right. \\
& \text { Singular } S_{12}(1)\left\{\begin{array}{l}
z_{1} \rightarrow(4.9+0.163 i)-(0.148-1.15 i) \epsilon+(0.803+3.69 i) \epsilon^{2} \\
z_{2} \rightarrow(4.9+0.163 i)-(2.89+0.276 i) \epsilon-(18.6-1.77 i) \epsilon^{2} \\
z_{3} \rightarrow(1.46-0.781 i)-(0.66+0.418 i) \epsilon-(3.94+1.17 i) \epsilon^{2} \\
z_{4} \rightarrow(8.4-0.412 i)+(20.3-13.4 i) \epsilon+(55.5-60.9 i) \epsilon^{2}
\end{array}\right. \\
& \text { Singular } S_{12}(2)\left\{\begin{aligned}
z_{1} & \rightarrow(4.99-0.209 i)-(0.974-1.16 i) \epsilon-(17 .+23.2 i) \epsilon^{2} \\
z_{2} & \rightarrow(4.99-0.209 i)+(1.95+2.3 i) \epsilon-(72.1+13 . i) \epsilon^{2} \\
z_{3} & \rightarrow(4.56-0.333 i)+(13.8-7.47 i) \epsilon+(44.9+397 . i) \epsilon^{2} \\
z_{4} & \rightarrow(3.47+1.82 i)+(4.5-2.73 i) \epsilon+(107 .+67.9 i) \epsilon^{2}
\end{aligned}\right. \\
& \text { Singular } S_{12}(3)\left\{\begin{array}{l}
z_{1} \rightarrow(4.94-0.54 i)-(3.24-0.525 i) \epsilon+(11.7-45.7 i) \epsilon^{2} \\
z_{2} \rightarrow(4.94-0.54 i)+(3.78+3.17 i) \epsilon-(77.5+23.5 i) \epsilon^{2} \\
z_{3} \rightarrow(3.88-0.729 i)+(28.6-16.3 i) \epsilon+(208 .+725 . i) \epsilon^{2} \\
z_{4} \rightarrow(1.99+3.72 i)-(1.77-7.54 i) \epsilon-(39.6+131 . i) \epsilon^{2}
\end{array}\right. \\
& \text { Singular } S_{12}(4)\left\{\begin{array}{l}
z_{1} \rightarrow(4.81-0.807 i)-(2.27+1.54 i) \epsilon-(13.8+5.69 i) \epsilon^{2} \\
z_{2} \rightarrow(4.81-0.807 i)+(7.88-1.43 i) \epsilon+(28.9-7.46 i) \epsilon^{2} \\
z_{3} \rightarrow(2.35+0.69 i)-(3.98-0.141 i) \epsilon-(15.2-5.13 i) \epsilon^{2} \\
z_{4} \rightarrow(1.46+5.15 i)-(10.9-10 . i) \epsilon-(44.2-60.1 i) \epsilon^{2}
\end{array}\right.
\end{aligned}
$$

There are two common singular solutions and six solutions for $S_{12}$ only. The quantity and asymptotic behavior are same as our analysis. The measure part is shown in Table XII. 
TABLE XII. Measure part of each singular solution of $S_{123}=S_{12}=0$.

\begin{tabular}{lc}
\hline \hline Solution & Measure \\
\hline Singular $S_{123} S_{12}(1)$ & $\left(691000 .-\left(5.97 \times 10^{6}\right) i\right) \epsilon^{4}+O\left(\epsilon^{5}\right)$ \\
Singular $S_{123} S_{12}(2)$ & $-\left(3.53 \times 10^{9}-\left(2.51 \times 10^{9}\right) i\right) \epsilon^{4}+O\left(\epsilon^{5}\right)$ \\
Singular $S_{12}(1)$ & $-(50.8+792 . i) \epsilon+O\left(\epsilon^{2}\right)$ \\
Singular $S_{12}(2)$ & $(158 .+104 . i) \epsilon+O\left(\epsilon^{2}\right)$ \\
Singular $S_{12}(3)$ & $-(27300 .+65900 . i) \epsilon+O\left(\epsilon^{2}\right)$ \\
Singular $S_{12}(4)$ & $(153000 .+66900 . i) \epsilon+O\left(\epsilon^{2}\right)$ \\
All Regulars & $-\left(84300 .+\left(3.87 \times 10^{6}\right) i\right)+\left(3.42 \times 10^{7}-\left(1.04 \times 10^{7}\right) i\right) \epsilon+O\left(\epsilon^{2}\right)$ \\
\hline \hline
\end{tabular}

TABLE XIII. Contribution of each singular solution of $I_{1}$.

\begin{tabular}{lc}
\hline \hline Solution & Contribution \\
\hline Singular $S_{123} S_{12}(1)$ & $-\frac{5.93 \times 10^{-7}+\left(6.32 \times 10^{-7}\right) i}{\epsilon}+O\left(\epsilon^{0}\right)$ \\
Singular $S_{123} S_{12}(2)$ & $\frac{1.62 \times 10^{-6}+\left(4.45 \times 10^{-7}\right) i}{\epsilon}+O\left(\epsilon^{0}\right)$ \\
Singular $S_{12}(1)$ & $1.17 \times 10^{-8}+\left(5.19 \times 10^{-9}\right) i$ \\
Singular $S_{12}(2)$ & $0.0000351+\left(6.06 \times 10^{-6}\right) i$ \\
Singular $S_{12}(3)$ & $-0.0000613+\left(6.88 \times 10^{-6}\right) i$ \\
Singular $S_{12}(4)$ & $-2.07 \times 10^{-7}+\left(2.57 \times 10^{-6}\right) i$ \\
All Regulars & $-(0.000127+0.0000511 i)-(0.0426+0.0299 i) \epsilon+O\left(\epsilon^{2}\right)$ \\
\hline \hline
\end{tabular}

The measure part of common singular solutions is exact $\epsilon^{2|A|+2|B|-6}=\epsilon^{2 \times 2+2 \times 3-6}=\epsilon^{4}$, and $\epsilon^{2|A|-3}=\epsilon^{2 \times 2-3}=\epsilon^{1}$ for the solutions only for pole $S_{12}$.

The first integrand we have chosen is

$$
I_{1}=-\frac{1}{z_{12} z_{13} z_{14} z_{17} z_{23}^{2} z_{27} z_{34} z_{45}^{2} z_{56}^{2} z_{67}^{2}}
$$

with pole index $\chi(123)=0, \chi(12)=-1$. The contribution of each solution is in Table XIII. The summation is

$$
\frac{1.03 \times 10^{-6}-\left(1.88 \times 10^{-7}\right) i}{\epsilon}+O\left(\epsilon^{0}\right)
$$

We verify the summation with numerical kinematics

$$
\begin{gathered}
-\frac{1}{S_{23} S_{45} S_{67} S_{123}}-\frac{1}{S_{23} S_{45} S_{123} S_{456}}-\frac{1}{S_{23} S_{56} S_{123} S_{456}}-\frac{1}{S_{23} S_{56} S_{123} S_{567}}-\frac{1}{S_{23} S_{67} S_{123} S_{567}} \\
=\frac{1.03 \times 10^{-6}-\left(1.88 \times 10^{-7}\right) i}{\epsilon}+\left(7.19 \times 10^{-7}-\left(5.16 \times 10^{-6}\right) i\right)+O(\epsilon)
\end{gathered}
$$

The second integrand is

$$
-\frac{1}{z_{14}^{2} z_{16}^{2} z_{23}^{2} z_{25}^{2} z_{34} z_{37} z_{47} z_{56} z_{57} z_{67}}
$$

with pole index $\chi(123)=-2, \chi(12)=-2$. The contribution of each solution is in Table XIV. The summation is

$$
-(0.000476-0.0000642 i)-(0.0000339+0.0000789 i) \epsilon+O\left(\epsilon^{2}\right) .
$$

We verify the summation with numerical kinematics:

$$
\begin{aligned}
& -\frac{1}{S_{14} S_{23} S_{146} S_{235}}-\frac{1}{S_{16} S_{23} S_{146} S_{235}}-\frac{1}{S_{14} S_{25} S_{146} S_{235}}-\frac{1}{S_{16} S_{25} S_{146} S_{235}} \\
& =-(0.000476-0.0000642 i)-(0.0000339+0.0000789 i) \epsilon+O\left(\epsilon^{2}\right) .
\end{aligned}
$$


TABLE XIV. Contribution of each singular solution of $I_{2}$.

\begin{tabular}{lc}
\hline \hline Solution & Contribution \\
\hline Singular $S_{123} S_{12}(1)$ & $-(0.00292-0.0564 i) \epsilon^{2}+O\left(\epsilon^{3}\right)$ \\
Singular $S_{123} S_{12}(2)$ & $(0.0703-0.0516 i) \epsilon^{2}+O\left(\epsilon^{3}\right)$ \\
Singular $S_{12}(1)$ & $-\left(0.0000246+\left(3.35 \times 10^{-6}\right) i\right) \epsilon+O\left(\epsilon^{2}\right)$ \\
Singular $S_{12}(2)$ & $(0.00186-0.000129 i) \epsilon+O\left(\epsilon^{2}\right)$ \\
Singular $S_{12}(3)$ & $-(0.00148+0.00198 i) \epsilon+O\left(\epsilon^{2}\right)$ \\
Singular $S_{12}(4)$ & $-(0.000134-0.000217 i) \epsilon+O\left(\epsilon^{2}\right)$ \\
All Regulars & $-(0.000476-0.0000642 i)-(0.000257-0.00181 i) \epsilon+O\left(\epsilon^{2}\right)$ \\
\hline \hline
\end{tabular}

From the result of these two integrands, we verify the contribution of common singular solution is $\epsilon^{-2-\chi(12)-\chi(123)}$, and $\epsilon^{-1-\chi(12)}$ for singular solutions of $S_{12}$.

Example III: $n=7, S_{123}=S_{234}=0$

Now we consider the case two poles are not compatible with each other, we choose $A=\{123\}$ and $B=\{234\}$. The numerical kinematics are

$$
\begin{aligned}
k_{1} \rightarrow & \{-0.198-0.128 i,-0.0155-4.35 i,-3.65+0.112 i,-0.829-0.0698 i,-2.23-0.138 i\} \\
& +\{0.16 \epsilon,(0.0591+0.504 i) \epsilon,(0.499-0.06 i) \epsilon,(0.0301+0.00534 i) \epsilon, 0.172 \epsilon\} \\
k_{2} \rightarrow & \{1.54,0.667,0.963,0.368,-0.931\} \\
k_{3} \rightarrow & \{1.17,-0.525,-0.27,0.459,-0.902\} \\
k_{4} \rightarrow & \{0.524-0.123 i, 0.1+0.999 i, 0.406-0.0327 i, 0.343-0.73 i,-1.23-0.0812 i\} \\
& +\{0.839 \epsilon,(-0.951-0.84 i) \epsilon,(0.187+0.00511 i) \epsilon,(-0.942+0.85 i) \epsilon, 0.552 \epsilon\} \\
k_{5} \rightarrow & \{0.951,-0.376,-0.594,0.164,-0.618\} \\
k_{6} \rightarrow & \{-1.17+0.123 i, 0.325-0.999 i, 1.23+0.0327 i, 0.0673+0.73 i, 1.12+0.0812 i\} \\
& +\{-0.839 \epsilon,(0.951+0.84 i) \epsilon,(-0.187-0.00511 i) \epsilon,(0.942-0.85 i) \epsilon,-0.552 \epsilon\} \\
k_{7} \rightarrow & \{-2.82+0.128 i,-0.176+4.35 i, 1.92-0.112 i,-0.573+0.0698 i, 4.78+0.138 i\} \\
& +\{-0.16 \epsilon,(-0.0591-0.504 i) \epsilon,(-0.499+0.06 i) \epsilon,(-0.0301-0.00534 i) \epsilon,-0.172 \epsilon\}
\end{aligned}
$$

We fix $z_{5}=1, z_{6}=5, z_{7}=-7$, the singular solutions are

$$
\begin{gathered}
\text { Singular } S_{123}(1)\left\{\begin{array}{l}
z_{1} \rightarrow(-6.59+5.61 i)+(0.988-7.57 i) \epsilon+(13.1+2.66 i) \epsilon^{2} \\
z_{2} \rightarrow(-6.59+5.61 i)+(0.622-8.5 i) \epsilon+(16.5+10.3 i) \epsilon^{2} \\
z_{3} \rightarrow(-6.59+5.61 i)+(0.143-8.57 i) \epsilon+(21.1+11 . i) \epsilon^{2} \\
z_{4} \rightarrow(5.71+5.41 i)-(2.95-11.1 i) \epsilon-(12 .+9.39 i) \epsilon^{2}
\end{array}\right. \\
\text { Singular } S_{123}(2)\left\{\begin{array}{l}
z_{1} \rightarrow(-5.2-0.937 i)-(0.505+0.588 i) \epsilon-(1.03+0.394 i) \epsilon^{2} \\
z_{2} \rightarrow(-5.2-0.937 i)-(0.839+0.422 i) \epsilon-(0.412+0.42 i) \epsilon^{2} \\
z_{3} \rightarrow(-5.2-0.937 i)-(0.852+0.241 i) \epsilon-(0.226+0.714 i) \epsilon^{2} \\
z_{4} \rightarrow(1.42-1.1 i)-(3.76+0.476 i) \epsilon-(2.6+0.484 i) \epsilon^{2}
\end{array}\right.
\end{gathered}
$$


TABLE XV. Measure part of each singular solution of $S_{123}=S_{234}=0$.

\begin{tabular}{lc}
\hline \hline Solution & Measure \\
\hline Singular $S_{123}(1)$ & $\left(6.05 \times 10^{6}-\left(7.15 \times 10^{6}\right) i\right) \epsilon^{3}+O\left(\epsilon^{4}\right)$ \\
Singular $S_{123}(2)$ & $(1020 .+829 . i) \epsilon^{3}+O\left(\epsilon^{4}\right)$ \\
Singular $S_{234}(1)$ & $\left(4.46 \times 10^{8}+\left(2.5 \times 10^{8}\right) i\right) \epsilon^{3}+O\left(\epsilon^{4}\right)$ \\
Singular $S_{234}(2)$ & $\left(2.8 \times 10^{9}+\left(6.32 \times 10^{9}\right) i\right) \epsilon^{3}+O\left(\epsilon^{4}\right)$ \\
All Regulars & $-\left(1.62 \times 10^{11}+\left(2.12 \times 10^{11}\right) i\right)+\left(5.05 \times 10^{11}+\left(8.03 \times 10^{12}\right) i\right) \epsilon+O\left(\epsilon^{2}\right)$ \\
\hline \hline
\end{tabular}

$$
\begin{gathered}
\text { Singular } S_{234}(1)\left\{\begin{array}{l}
z_{1} \rightarrow(0.948+9 . i)-(14.1+3.67 i) \epsilon-(40.7+39.5 i) \epsilon^{2} \\
z_{2} \rightarrow(6.25+3.19 i)-(0.79-13.5 i) \epsilon-(31.8-47.6 i) \epsilon^{2} \\
z_{3} \rightarrow(6.25+3.19 i)-(3.64-7.5 i) \epsilon-(32.8-22.1 i) \epsilon^{2} \\
z_{4} \rightarrow(6.25+3.19 i)+(0.0579+8.31 i) \epsilon-(20.6-16.6 i) \epsilon^{2}
\end{array}\right. \\
\text { Singular } S_{234}(2)\left\{\begin{array}{l}
z_{1} \rightarrow(-6.25-0.445 i)+(7.43+7.67 i) \epsilon+(11.3-45.2 i) \epsilon^{2} \\
z_{2} \rightarrow(-2.7-1.43 i)-(4.19-3.88 i) \epsilon+(37.1+13.9 i) \epsilon^{2} \\
z_{3} \rightarrow(-2.7-1.43 i)+(28.6+19.4 i) \epsilon-(9.49+134 . i) \epsilon^{2} \\
z_{4} \rightarrow(-2.7-1.43 i)+(10.2+28.8 i) \epsilon+(49.1-127 . i) \epsilon^{2}
\end{array}\right.
\end{gathered}
$$

The quantity and asymptotic behavior is conform with our analysis. The measure part given in the Table XV shows $\epsilon^{2|A|-3}=\epsilon^{2|B|-3}=\epsilon^{2 \times 3-3}=\epsilon^{3}$, which fit our analysis.

We choose the first integrand as

$$
I_{1}=-\frac{1}{z_{12} z_{13} z_{14} z_{17} z_{23}^{2} z_{27} z_{34} z_{45}^{2} z_{56}^{2} z_{67}^{2}}
$$

with pole index $\chi(123)=0, \chi(234)=-1$. The contribution of each singular solution is in Table XVI. The summation

$$
\frac{0.188-0.277 i}{\epsilon}+O\left(\epsilon^{0}\right)
$$

and can be verified with the amplitude after putting the kinematics

$$
\begin{gathered}
-\frac{1}{S_{23} S_{45} S_{67} S_{123}}-\frac{1}{S_{23} S_{45} S_{123} S_{456}}-\frac{1}{S_{23} S_{56} S_{123} S_{456}}-\frac{1}{S_{23} S_{56} S_{123} S_{567}}-\frac{1}{S_{23} S_{67} S_{123} S_{567}} \\
=\frac{0.188-0.277 i}{\epsilon}+(1.78-0.469 i)+(6.24+3.76 i) \epsilon+O\left(\epsilon^{2}\right) .
\end{gathered}
$$

We choose the second integrand as

$$
I_{2}=-\frac{1}{z_{12} z_{15} z_{17}^{2} z_{23}^{2} z_{25} z_{34}^{2} z_{45} z_{46} z_{56} z_{67}^{2}}
$$

TABLE XVI. The contribution of each singular solution of $I_{1}$.

\begin{tabular}{lc}
\hline \hline Solution & Integration \\
\hline Singular $S_{123}(1)$ & $\frac{0.00281+0.054 i}{\epsilon}+O\left(\epsilon^{0}\right)$ \\
Singular $S_{123}(2)$ & $\frac{0.185-0.331 i}{\epsilon}+O\left(\epsilon^{0}\right)$ \\
Singular $S_{234}(1)$ & $0.0000327+0.000439 i$ \\
Singular $S_{234}(2)$ & $-0.00337+0.0353 i$ \\
All Regulars & $-(0.0143+0.0791 i)+(1.62-0.868 i) \epsilon+O\left(\epsilon^{2}\right)$ \\
\hline \hline
\end{tabular}


TABLE XVII. The contribution of each singular solution of $I_{2}$.

\begin{tabular}{lc}
\hline \hline Solution & Contribution \\
\hline Singular $S_{123}(1)$ & $(0.00415-0.000281 i)+O\left(\epsilon^{1}\right)$ \\
Singular $S_{123}(2)$ & $-(0.00517-0.0029 i)+O\left(\epsilon^{1}\right)$ \\
Singular $S_{234}(1)$ & $-\frac{0.000857-0.00062 i}{\epsilon}+O\left(\epsilon^{0}\right)$ \\
Singular $S_{234}(2)$ & $-\frac{0.0000737+0.00856 i}{\epsilon}+O\left(\epsilon^{0}\right)$ \\
All Regulars & $-(0.241-0.0685 i)-(8.06+9.33 i) \epsilon+O\left(\epsilon^{2}\right)$ \\
\hline \hline
\end{tabular}

with pole index $\chi(123)=-1, \chi(234)=0$. The contribution of each singular solution is in Table XVII. The summation is

$$
-\frac{0.000931+0.00794 i}{\epsilon}+O\left(\epsilon^{0}\right)
$$

which are the same as the amplitude after putting the kinematics

$$
\begin{aligned}
- & \frac{1}{S_{17} S_{23} S_{167} S_{234}}-\frac{1}{S_{17} S_{34} S_{167} S_{234}}-\frac{1}{S_{23} S_{67} S_{167} S_{234}}-\frac{1}{S_{34} S_{67} S_{167} S_{234}} \\
& =-\frac{0.000931+0.00794 i}{\epsilon}+(0.00343-0.0000467 i)-(0.0108+0.0421 i) \epsilon+O\left(\epsilon^{2}\right) .
\end{aligned}
$$

We choose the third integrand as

$$
I_{3}=\frac{1}{z_{12}^{2} z_{15} z_{17} z_{23}^{2} z_{34}^{2} z_{45} z_{47} z_{56}^{2} z_{67}^{2}}
$$

with pole index $\chi(123)=\chi(234)=0$. The contribution of each singular solution is in Table XVIII. The summation is

$$
-\frac{0.0424+0.0109 i}{\epsilon}+O\left(\epsilon^{0}\right)
$$

and the amplitude after putting the kinematics is

$$
\begin{aligned}
& \frac{1}{S_{12} S_{34} S_{56} S_{567}}+\frac{1}{S_{12} S_{34} S_{67} S_{567}}+\frac{1}{S_{12} S_{56} S_{123} S_{567}}+\frac{1}{S_{23} S_{56} S_{123} S_{567}}+\frac{1}{S_{12} S_{67} S_{123} S_{567}} \\
& +\frac{1}{S_{23} S_{67} S_{123} S_{567}}+\frac{1}{S_{23} S_{56} S_{234} S_{567}}+\frac{1}{S_{34} S_{56} S_{234} S_{567}}+\frac{1}{S_{23} S_{67} S_{234} S_{567}}+\frac{1}{S_{34} S_{67} S_{234} S_{567}} \\
& \quad=-\frac{0.0424+0.0109 i}{\epsilon}-(0.154+0.0133 i)-(0.397-0.0194 i) \epsilon+O\left(\epsilon^{2}\right) .
\end{aligned}
$$

We found that the contribution of each singular solution is exact $\epsilon^{-1-\chi(A)}$ and $\epsilon^{-1-\chi(B)}$ respectively.

TABLE XVIII. The contribution of each singular solution of $I_{3}$.

\begin{tabular}{lc}
\hline \hline Solution & Contribution \\
\hline Singular $S_{123}(1)$ & $-\frac{0.0177+0.0131 i}{\epsilon}+O\left(\epsilon^{0}\right)$ \\
Singular $S_{123}(2)$ & $-\frac{0.0223+0.00101 i}{\epsilon}+O\left(\epsilon^{0}\right)$ \\
Singular $S_{234}(1)$ & $-\frac{0.000521-0.000342 i}{\epsilon}+O\left(\epsilon^{0}\right)$ \\
Singular $S_{234}(2)$ & $-\frac{0.00196-0.00286 i}{\epsilon}+O\left(\epsilon^{0}\right)$ \\
All Regulars & $(0.1+0.0492 i)+(0.903+5.43 i) \epsilon+O\left(\epsilon^{2}\right)$ \\
\hline \hline
\end{tabular}




\section{FROM SINGULAR KINEMATICS TO SOLUTIONS II: THE SOFT LIMIT}

Now we consider the second type of singularity, the soft limit, for example, the $k_{1} \rightarrow 0$. Different from the preview case, when $k_{1} \rightarrow 0$, we have $S_{1 i} \rightarrow 0$ for all $i=2, \ldots, n$ automatically. If we naively use the picture from the previous section, we will conclude that there will be some singular solutions with $z_{2} \rightarrow z_{1}$ and some other solutions with $z_{3} \rightarrow z_{1}$, etc. Since poles $S_{1 i}$ are not compatible, the singular solutions will be the union of all these $z_{i} \rightarrow z_{1}$. However, as will be shown in this section, the above naive picture is wrong. In fact, for the soft limit, there is no singular solution.

With the behavior $k_{1}=k_{1}^{(1)} \epsilon$ and $k_{a \neq 1}^{(0)} \neq 0$ when $\epsilon \rightarrow 0$, the scattering equation $\mathcal{E}_{1}$ is

$$
\mathcal{E}_{1}=\epsilon\left[\sum_{a \in\{2, \ldots, N\}} \frac{k_{1}^{(1)} \cdot k_{a}^{(0)}}{z_{1}-z_{a}}+\mathcal{O}(\epsilon)\right]=0
$$

Assuming there is at most one location, for example, $z_{2} \rightarrow z_{1}$ while other $z_{i}$ 's are separated from each other, the term $\frac{k_{1}^{(1)} \cdot k_{2}^{(0)}}{z_{1}-z_{2}}$ in (4.1) will be singular comparing to other terms, since in general $k_{a}^{(0)} \neq 0, k_{1}^{(1)} \cdot k_{a}^{(0)} \neq 0$ and all numerators in (4.1) are nonzero. Thus (4.1) could not be satisfied under above assumption.

There are two possibilities to make (4.1) true. The first possibility is that there are at least two locations, for example, $z_{i}, z_{j} \rightarrow z_{1}$. Then by our careful analysis given in Sec. II, we will have $S_{A} \rightarrow 0$ with $3 \leq|A| \leq n-3$, which conflicts with our kinematic condition that under $k_{1} \rightarrow 0$, only $S_{1 i} \rightarrow 0$. Thus there is left with only another possibility, i.e., all $z_{i}$ 's are separated from each other and $z_{1}$, so all terms in (4.1) will have equivalent weight to make it zero.

Having proved that there is no singular solution for scattering equations, we need to understand how soft singularities are produced in the amplitude. The key is the measure part. Unlike the one $J \sim \epsilon^{2|A|-3} \rightarrow 0$ given in (5.4), for soft limit

$$
\begin{aligned}
\left(z_{1}-z_{2}\right)\left(z_{2}-z_{3}\right)\left(z_{3}-z_{1}\right)\left(z_{n}-z_{n-1}\right)\left(z_{n-1}-z_{n-2}\right)\left(z_{n-2}-z_{n}\right) & \sim \epsilon^{0} \\
|\Phi|_{i j k}^{r s t} \rightarrow \epsilon^{1}, \quad J & \sim \epsilon^{-1}
\end{aligned}
$$

Thus it is the measure providing the singularity.

Now we present an example to demonstrate the behavior of soft limit. First we construct the kinematics for the soft limit. According to the discussion given in the Appendix, we construct $\tilde{p}_{i}, i=2, \ldots, n$ such that $\sum_{i=2}^{n} \tilde{p}_{i}=0$, then we choose $u$, $v$ such that

$$
p_{1}=\epsilon(u+v), \quad p_{2}=\tilde{p}_{2}-\epsilon u \quad p_{3}=\tilde{p}_{3}-\epsilon v, \quad p_{a}=\tilde{p_{a}}, \quad a=4, \ldots, n
$$

The $u$ and $v$ are not arbitrary. To make all momenta on-shell, they need to satisfy

$$
u \cdot v=0 \quad u^{2}=v^{2}=0, \quad u \cdot \tilde{p}_{2}=0, \quad v \cdot \tilde{p}_{3}=0
$$

For space-time dimension $D \geq 4$, there are solutions for $u, v$.

Using above frame, let us present one example, i.e., the six point case with following choice of kinematics

$$
\begin{aligned}
k_{1} \rightarrow & \{0.774,-2.039+1.645 i,-2.358-2.948 i, 2.216-1.623 i,-0.0675\} \epsilon, \\
k_{2} \rightarrow & \{1.125,0.595,-0.105,0.802,0.507\} \\
& +\{-0.162,-0.876,-0.059-0.969 i, 0.454-0.127 i,-0.0628 i\} \epsilon, \\
k_{3} \rightarrow & \{0.950,-0.683,-0.0312,-0.572,-0.329\} \\
& +\{-0.612,2.915-1.645 i, 2.417+3.917 i,-2.668+1.750 i, 0.130\} \epsilon \\
k_{4} \rightarrow & \{1.17,-0.617,-0.284,-0.951,0.0575\}, \\
k_{5} \rightarrow & \{-1.247,-0.299,0.288,-0.936,-0.71\}, \\
k_{6} \rightarrow & \{-1.999,1.003,0.132,1.66,0.475\}
\end{aligned}
$$


We fix the gauge choice $z_{4}=1, z_{5}=0, z_{6}=-1$ and find following six solutions

$$
\begin{gathered}
\left\{\begin{array}{l}
z_{1} \rightarrow(-0.236755-0.255543 i)-(1.47022-0.800113 i) \epsilon+O\left(\epsilon^{2}\right) \\
z_{2} \rightarrow(-0.0108264+0.011729 i)+(0.0134425+0.0210137 i) \epsilon+O\left(\epsilon^{2}\right) \\
z_{3} \rightarrow(-0.28117-0.303133 i)-(1.54701-0.967519 i) \epsilon+O\left(\epsilon^{2}\right)
\end{array}\right. \\
\left\{\begin{array}{l}
z_{1} \rightarrow(-0.00979118+0.011149 I)-(0.0327251+0.0713408 I) \epsilon+O\left(\epsilon^{2}\right) \\
z_{2} \rightarrow(-0.0108264+0.011729 i)+(0.0134425+0.0210137 i) \epsilon+O\left(\epsilon^{2}\right) \\
z_{3} \rightarrow(-0.28117-0.303133 i)-(1.54701-0.967519 i) \epsilon+O\left(\epsilon^{2}\right)
\end{array}\right. \\
\left\{\begin{array}{l}
z_{1} \rightarrow(0.261008+0.153645 i)-(0.587951-0.0543757 i) \epsilon+O\left(\epsilon^{2}\right) \\
z_{2} \rightarrow(-0.0108264+0.011729 i)+(0.0134425+0.0210137 i) \epsilon+O\left(\epsilon^{2}\right) \\
z_{3} \rightarrow(-0.28117-0.303133 i)-(1.54701-0.967519 i) \epsilon+O\left(\epsilon^{2}\right)
\end{array}\right. \\
\left\{\begin{array}{l}
z_{1} \rightarrow(-0.19289+0.33945 i)-(0.36536+0.659837 i) \epsilon+O\left(\epsilon^{2}\right) \\
z_{2} \rightarrow(-0.0108264-0.011729 i)+(0.017517+0.0426134 i) \epsilon+O\left(\epsilon^{2}\right) \\
z_{3} \rightarrow(-0.28117+0.303133 i)-(0.225438+0.604153 i) \epsilon+O\left(\epsilon^{2}\right)
\end{array}\right. \\
\left\{\begin{array}{l}
z_{1} \rightarrow(-0.010308-0.0107239 i)+(0.130698+0.227897 i) \epsilon+O\left(\epsilon^{2}\right) \\
z_{2} \rightarrow(-0.0108264-0.011729 i)+(0.017517+0.0426134 i) \epsilon+O\left(\epsilon^{2}\right) \\
z_{3} \rightarrow(-0.28117+0.303133 i)-(0.225438+0.604153 i) \epsilon+O\left(\epsilon^{2}\right) \\
z_{2} \rightarrow(-0.0108264-0.011729 i)+(0.017517+0.0426134 i) \epsilon+O\left(\epsilon^{2}\right) \\
z_{3} \rightarrow(-0.28117+0.303133 i)-(0.225438+0.604153 i) \epsilon+O\left(\epsilon^{2}\right)
\end{array}\right.
\end{gathered}
$$

We have presented these six solutions into two groups. When checking carefully, one can see that the values of $z_{2}, z_{3}$ are the same in each group. In fact, as shown in [7], for the general case of $n$ points with soft limit $k_{1} \rightarrow 0$, all ( $\left.n-3\right)$ ! solutions can divided into $(n-4)$ ! groups: in each group the values of $z_{i}, i=2,3, \ldots, n$ are same while the $(n-3)$ values of $z_{1}$ are different. More explicitly, at the leading order, the scattering equations become

$$
\mathcal{E}_{a}=\sum_{b=2, b \neq a}^{n} \frac{k_{a} \cdot k_{b}}{z_{a}-z_{b}}
$$

for $a=2,3, \ldots, n$ and

$$
\mathcal{E}_{1}=\epsilon\left[\sum_{a \in\{2, \ldots, N\}} \frac{k_{1}^{(1)} \cdot k_{a}^{(0)}}{z_{1}-z_{a}}+\mathcal{O}(\epsilon)\right]=0
$$

Using (4.8) we get $(n-4)$ ! different solutions for $z_{i}, i=2,3, \ldots, n$. Putting them to (4.9) we get $(n-3)$ solutions for $z_{1}$. Using (4.6) and (4.7) the measure $J=\frac{z_{12} z_{23} z_{31} z_{45} z_{56} z_{64}}{|\Phi|_{456}^{123}}$ of each solution is

$$
\begin{aligned}
& J_{1}=-\frac{8.28657 \times 10^{-6}+5.97512 \times 10^{-6} i}{\epsilon}-(0.00010047-0.000143733 i)+\mathcal{O}(\epsilon) \\
& J_{2}=\frac{9.27966 \times 10^{-9}+1.71473 \times 10^{-8} i}{\epsilon}+\left(6.26664 \times 10^{-7}-5.07552 \times 10^{-9} i\right)+\mathcal{O}(\epsilon) \\
& J_{3}=-\frac{0.0000888246+0.0000219678 i}{\epsilon}-(0.00413616-0.00188736 i)+\mathcal{O}(\epsilon)
\end{aligned}
$$




$$
\begin{aligned}
& J_{4}=-\frac{7.12674 \times 10^{-6}+0.0000270679 i}{\epsilon}+(0.000134457+0.000191776 i)+\mathcal{O}(\epsilon) \\
& J_{5}=\frac{1.73122 \times 10^{-8}-4.0953 \times 10^{-9} i}{\epsilon}-\left(5.04811 \times 10^{-7}+1.2758 \times 10^{-7} i\right)+\mathcal{O}(\epsilon) \\
& J_{6}=\frac{0.0000631231-0.0000764316 i}{\epsilon}-(0.0000541201+0.00243992 i)+\mathcal{O}(\epsilon)
\end{aligned}
$$

where each one has the leading behavior $\frac{1}{\epsilon}$ indeed. Now we choose two different CHY-integrands: one contains pole $S_{1 a}$ and another does not.

(i) $I_{\mathrm{CHY}}=\frac{1}{z_{12}^{2} z_{23} z_{25} z_{34}^{2} z_{41} z_{45} z_{56}^{2} z_{61} z_{63}}$ with pole $S_{12}$. The analytic result gives

$$
\frac{1}{S_{12} S_{34} S_{56}}=\frac{1.83042-0.257211 i}{\epsilon}+(6.21704-21.8651 i)+O(\epsilon)
$$

after substituting (4.5). The contributions of each solution are

$$
\begin{aligned}
S_{1} & : \frac{0.00419196-0.00814611 i}{\epsilon}-(0.00751988-0.0171901 i)+O(\epsilon) \\
S_{2} & : \frac{0.635358-1.40928 i}{\epsilon}-(18.0379+34.6097 i)+O(\epsilon) \\
S_{3} & : \frac{0.0838495-0.0761965 i}{\epsilon}-(0.293397+2.66308 i)+O\left(\epsilon^{2}\right) \\
S_{4} & : \frac{0.0173534+0.0060881 i}{\epsilon}+(0.0108633+0.0298797 i)+O(\epsilon) \\
S_{5} & : \frac{1.05731+1.13749 i}{\epsilon}+(23.2755+14.1021 i)+O(\epsilon) \\
S_{6} & : \frac{0.0670696+0.105014 i}{\epsilon}+(1.26947+1.25845 i)+O(\epsilon) \\
\sum_{i=1}^{6} S_{i}: & \frac{1.83042-0.257211 i}{\epsilon}+(6.21704-21.8651 i)+O(\epsilon)
\end{aligned}
$$

(ii) $I_{\mathrm{CHY}}=\frac{1}{z_{12} z_{13} z_{23} z_{25} z_{32} z_{34} z_{41} z_{45} z_{56}^{2} z_{61} z_{64}}$ without the pole $S_{1 a}$. The analytic expression gives

$$
\frac{1}{S_{23} S_{56} S_{123}}=-0.00611146-(0.00176926+0.000337693 i) \epsilon+O\left(\epsilon^{2}\right)
$$

which is not divergent when $\epsilon \rightarrow 0$. The contributions of each solution are

$$
\begin{aligned}
& S_{1}: \frac{0.0294162+0.0533801 i}{\epsilon}-(0.15429+0.0531782 i)-(0.399708-0.492001 i) \epsilon+O\left(\epsilon^{2}\right) \\
& S_{2}:-\frac{0.00502886-0.00214344 i}{\epsilon}-(0.0153742+0.0794493 i)+(3.43002+0.885407 i) \epsilon+O\left(\epsilon^{2}\right) \\
& S_{3}:-\frac{0.0243873+0.0555235 i}{\epsilon}+(0.166609+0.697732 i)-(37.8097+16.3499 i) \epsilon+O\left(\epsilon^{2}\right) \\
& S_{4}: \frac{0.0936548-0.0110378 i}{\epsilon}-(0.0469898+0.113523 i)+(0.563917-0.0892481 i) \epsilon+O\left(\epsilon^{2}\right)
\end{aligned}
$$




$$
\begin{gathered}
S_{5}:-\frac{0.00413562+0.00322131 i}{\epsilon}-(0.0000649134-0.0756482 i)-(0.337054-0.169771 i) \epsilon+O\left(\epsilon^{2}\right) \\
S_{6}:-\frac{0.0895192-0.0142591 i}{\epsilon}+(0.043999-0.527229 i)+(2.47751+42.7249 i) \epsilon+O\left(\epsilon^{2}\right) \\
\sum_{i=1}^{6} S_{i}:-0.00611146-(0.00176926+0.000337693 i) \epsilon+O\left(\epsilon^{2}\right)
\end{gathered}
$$

For this CHY-integrand, the critical point is how the cancellation of $\frac{1}{\epsilon}$ part happens. When checking carefully, one can see the cancellation happens inside each group, i.e., for $S_{1}+S_{2}+S_{3}$ and $S_{4}+S_{5}+S_{6}$, the $\frac{1}{\epsilon}$ part disappears. This is a general phenomenon, and we can easily prove using the trick given in [7]:

$$
\begin{aligned}
A_{n}(1,2, \ldots, n) & =\int \prod_{i=1}^{n-3} d z_{i} z_{n-2, n-1}^{2} z_{n-1, n}^{2} z_{n, n-2}^{2} \prod_{a \neq n-2, n-1, n} \delta\left(\mathcal{E}_{a}\right) P T\left(1, \alpha_{2}, \ldots, \alpha_{n}\right) P T\left(1, \beta_{2}, \ldots, \beta_{n}\right) \\
& =\int \prod_{i=1}^{n-3} d z_{i} \delta\left(E_{1}\right) \frac{z_{\alpha_{n} \alpha_{2}}}{z_{1 \alpha_{2}} z_{1 \alpha_{n}}} \frac{z_{\beta_{n \beta_{2}}}}{z_{1 \beta_{2}} z_{1 \beta_{n}}} \prod_{\substack{a \neq 1, n-2, n-1, n}} \delta\left(\mathcal{E}_{a}\right) P T\left(\alpha_{2}, \ldots, \alpha_{n}\right) P T\left(\beta_{2}, \ldots, \beta_{n}\right) z_{n-2, n-1}^{2} z_{n-1, n}^{2} z_{n, n-2}^{2} \\
& =\int d z_{1} \delta\left(E_{1}\right) \frac{z_{\alpha_{n} \alpha_{2}}}{z_{1 \alpha_{2}} z_{1 \alpha_{n}}} \frac{z_{\beta_{n \beta_{2}}}}{z_{1 \beta_{2}} z_{1 \beta_{n}}} \int \prod_{i=2}^{n-3} d z_{i} \prod_{\substack{a \neq 1, n-2, n-1, n}} \delta\left(\mathcal{E}_{a}\right) P T\left(\alpha_{2}, \ldots, \alpha_{n}\right) P T\left(\beta_{2}, \ldots, \beta_{n}\right) z_{n-2, n-1}^{2} z_{n-1, n}^{2} z_{n, n-2}^{2} \\
& =\int d z_{1} \delta\left(E_{1}\right) \frac{z_{\alpha_{n} \alpha_{2}}}{z_{1 \alpha_{2}} z_{1 \alpha_{n}}} \frac{z_{\beta_{n \beta_{2}}}}{z_{1 \beta_{2}} z_{1 \beta_{n}}} A_{n-1}(2,3, \ldots, n)
\end{aligned}
$$

where at the leading part of $\epsilon$, the $\prod_{\substack{a \neq 1, n-2, n-1, n}} \delta\left(\mathcal{E}_{a}\right)$ takes the form $(4.8)$ so $A_{n-1}(2,3, \ldots, n)$ can be treated as a constant. To carry out the contour integration of $z_{1}$, using (4.9) we write $\mathcal{E}_{1}=\epsilon \sum_{i=2}^{n} \frac{k_{1}^{(1)} \cdot k_{i}^{(0)}}{z_{1}-z_{i}}=\epsilon \frac{f\left(z_{1} ; z_{2}, \ldots, z_{n}\right)}{z_{12} z_{13} \cdots z_{1 n}}$, thus

$$
\begin{aligned}
\int d z_{1} \delta\left(\mathcal{E}_{1}\right) \frac{z_{\alpha_{n} \alpha_{2}}}{z_{1 \alpha_{2}} z_{1 \alpha_{n}}} \frac{z_{\beta_{n \beta_{2}}}}{z_{1 \beta_{2}} z_{1 \beta_{n}}} & =\oint_{\Gamma_{f}} d z_{1} \frac{1}{\mathcal{E}_{1}} \frac{z_{\alpha_{n} \alpha_{2}}}{z_{1 \alpha_{2}} z_{1 \alpha_{n}}} \frac{z_{\beta_{n \beta_{2}}}}{z_{1 \beta_{2}} z_{1 \beta_{n}}} \\
& =\oint_{\Gamma_{f}} d z_{1} \frac{z_{12} z_{13} \cdots z_{1 n}}{\epsilon f\left(z_{1} ; z_{2}, \ldots, z_{n}\right)} \frac{z_{\alpha_{n} \alpha_{2}}}{z_{1 \alpha_{2}} z_{1 \alpha_{n}}} \frac{z_{\beta_{n \beta_{2}}}}{z_{1 \beta_{2}} z_{1 \beta_{n}}}
\end{aligned}
$$

where the contour $\Gamma_{f}$ means taking the sum only over poles coming from $f$. One can see that because the numerator $z_{12} z_{13} \cdots z_{1 n}$, as long as all $\alpha_{1}, \alpha_{n}, \beta_{2}, \beta_{n}$ are different, there is no extra pole in the denominator besides these coming from $f$, thus by the Cauchy residue theorem, ${ }^{9}$ the integral is zero, which is exact the case of the CHY-integrand containing no poles of $S_{1 a}$. On the other hand, if there are at least two labels of $\alpha_{2}, \alpha_{n}, \beta_{2}, \beta_{n}$ same, the integral is nonzero by the extra poles in the denominator, which corresponding to the CHY-integrand with pole $S_{1 a}$. One further point is that by (4.16), no matter if the $S_{1 a}$ is a single-pole or poles of higher degrees, the leading behavior is always $\frac{1}{\epsilon}$.

\section{FROM SINGULAR KINEMATICS TO SOLUTIONS III: THE FORWARD LIMIT}

The last singular kinematics we will discussed in the paper is the forward limit

$$
k_{1}+k_{2}=\epsilon q \rightarrow 0
$$

As we have mentioned in the section on motivation, the forward limit is very useful when discussing the loop-level CHY-integrands. In this section, we will give a general discussion of forward limit first, and then apply it to the one-loop CHY-integrals.

\footnotetext{
${ }^{9}$ The large $z_{1}$ behavior is $\frac{z_{1}^{n-1}}{z_{1}^{(n-3)+4}} \sim \frac{1}{z_{1}^{2}}$, so there is no boundary contribution at $z_{1}=\infty$.
} 


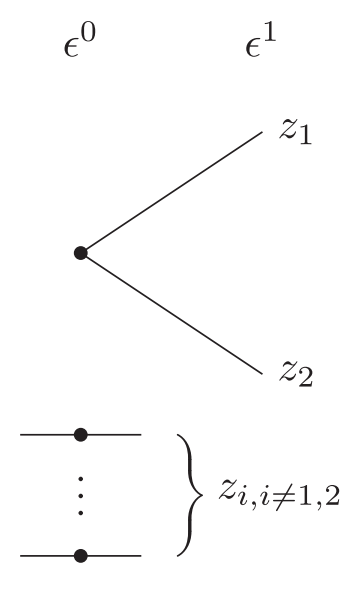

FIG. 5. Singular-I.

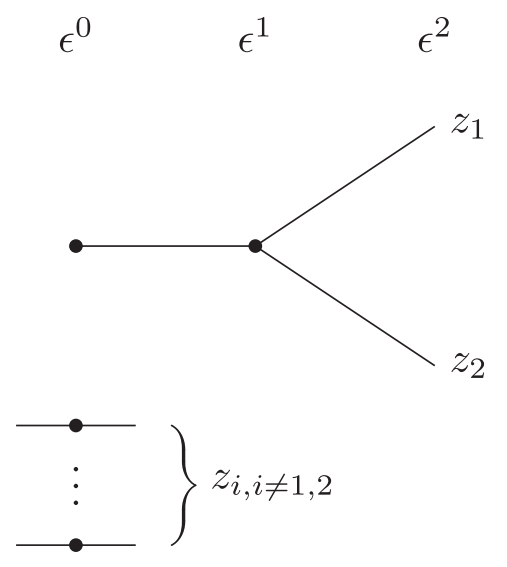

FIG. 6. Singular-II.

\section{A. The general discussion}

Similarly to the soft limit, under the limit (5.1) we will have $S_{12 i} \rightarrow 0$ for all $i=3, \ldots, n$ automatically under the forward limit (5.1). As we will see, the forward limit can be considered as the mixture of the factorization limit and the soft limit. While all poles $S_{12 i}$ are not compatible, all of them contain the common pole $S_{12} \rightarrow 0$. Now we ask what the implication of the singular kinematics to the is the behavior of solutions of scattering equations?

Let us start with the singular kinematics $S_{12} \rightarrow 0$. Unlike the factorization limit where $S_{A} \rightarrow \epsilon^{1}$, the (5.1) implies $S_{12} \rightarrow \epsilon^{2}$. The difference will lead to the different behavior of singular solutions, As given in [53], He and Yuan have shown that there are two kinds of singular solutions

$$
\begin{aligned}
\text { Singular }-\mathrm{I}:\left|z_{1}-z_{2}\right| \sim \epsilon^{1}, \\
\text { Singular }-\mathrm{II}:\left|z_{1}-z_{2}\right| \sim \epsilon^{2} .
\end{aligned}
$$

where each kind has $(n-4)$ ! solutions. In other words, like the factorization limit, the singular kinematics $S_{12} \rightarrow 0$ implies the singular solution. The counting of $(n-4)$ ! of each type matches the counting of factorization limit of pole $S_{12}$ too. However, unlike the factorization limit with only one type of singular solutions, there are two types of singular solutions (see Figs. 5 and 6), especially type II with the limit behavior $\left|z_{1}-z_{2}\right| \sim \epsilon^{2}{ }^{10}$.

Now we move to the automatic singular kinematics $S_{12 i} \rightarrow 0$. Do they imply the singular solution of $z_{1}, z_{2}, z_{i} \rightarrow z$ ? With the experience from the soft limit, we need to do more careful analysis. Assuming there is a singular solution where, for example, $z_{3}$ tend to $z_{1,2}$ while all other $z_{i}$ are separated from each other. In the scattering equation

$\mathcal{E}_{1}=\frac{k_{1} \cdot k_{2}}{z_{1}-z_{2}}+\frac{k_{1} \cdot k_{3}}{z_{1}-z_{3}}+\frac{k_{1} \cdot k_{4}}{z_{1}-z_{4}}+\cdots+\frac{k_{1} \cdot k_{n}}{z_{1}-z_{n}}=0$.

since $k_{1} \cdot k_{2}=\frac{\epsilon^{2}}{2} q$, term $\frac{k_{1} \cdot k_{2}}{z_{1}-z_{2}} \sim \epsilon^{1} / \epsilon^{0}$ depending if it is singular I or singular II solutions, while other terms $\frac{k_{1} \cdot k_{i}}{z_{1}-z_{i}} \sim \epsilon^{0}, i \neq 2,3$ because $k_{1} \cdot k_{i} \sim \epsilon^{0}$ and $\left|z_{1}-z_{i}\right| \sim \epsilon^{0}$. But the term $\frac{k_{1} \cdot k_{3}}{z_{1}-z_{3}}$ will be singular because $z_{3} \rightarrow z_{1}$, unless $S_{13}=2 k_{1} \cdot k_{3} \rightarrow 0$, which is in conflict with our kinematic configuration. Therefore, we learned that there is no other $z_{i}(i \neq 1,2)$ tend to $z_{1,2}$. The only possible asymptotic behavior of singular solutions must be (5.1). Now let us consider the contributions of singular solutions to amplitudes. We chose $(r s t)=(123)$ and $(i j k)=(n-2$, $n-1, n)$. For the measure part,

$$
\begin{aligned}
\left(z_{1}-z_{2}\right)\left(z_{2}-z_{3}\right)\left(z_{3}-z_{1}\right)\left(z_{n}-z_{n-1}\right)\left(z_{n-1}-z_{n-2}\right)\left(z_{n-2}-z_{n}\right) & \sim\left\{\begin{array}{ll}
\epsilon^{1}, & \text { Singular I } \\
\epsilon^{2}, & \text { Singular II }
\end{array}|\Phi|_{i j k}^{r s t} \sim \epsilon^{1}\right. \\
J & \sim \begin{cases}\epsilon^{0}, & \text { Singular I } \\
\epsilon^{1}, & \text { Singular II }\end{cases}
\end{aligned}
$$

\footnotetext{
${ }^{10} \mathrm{~A}$ naive understanding is that (5.1) implies there are two kinematic singularities simultaneously. The first one is the $\left(k_{1}+k_{2}\right) \rightarrow \epsilon q$, which is the soft limit type. The second one is the $\left(k_{1}-k_{2}\right) \rightarrow 2 k_{1}$, which is more like the factorization limit.
} 
With above knowledge we summary the contributions of each type of solutions as follows One interesting point is that the singular I solutions will contribute $I_{\mathrm{CHY}} \sim \frac{1}{\left(z_{1}-z_{2}\right)^{0}}$ at the order $\epsilon^{0}$, just like the regular solutions. Thus we can not naively remove their contributions ${ }^{11}$

Having the above discussion, we present one numerical example to show the above conclusions. The choice of kinematics has been given in Appendix. We choose the six-point example since it is the simplest nontrivial case under the forward limit. The numerical kinematics is given by

$$
\begin{aligned}
k_{1} \rightarrow & \{0.824,-0.421,0.306,0.283,-0.572,0\} \\
k_{2} \rightarrow & \{1.169,0.705,0.291,0.341,-0.817,0\} \\
k_{3} \rightarrow & \{-0.677,0.284,0.136,-0.316,+0.509,0\} \\
& +\{-0.323,-0.387+0.282 i,-0.185-0.591 i,-0.320,0.496,0\} \epsilon \\
k_{4} \rightarrow & \{-1.315,-0.568,-0.733,-0.309,0.880,0\} \\
& +\{-0.714,0.417,0.0996,-0.443-1.142 i, 1.262-0.401 i, 0\} \epsilon \\
k_{+} \rightarrow & \{0.410,-0.158,0.186+0.0370 i,-0.0794+0.0870 i,-0.333,0\} \\
& +\{0.518,-0.0148-0.141 i, 0.043+0.295 i, 0.382+0.571 i,-0.879+0.201 i, 0.128-0.442 i\} \epsilon \\
k_{-} \rightarrow & -\{0.410,-0.158,0.186+0.0370 i,-0.0794+0.0870 i,-0.333,0\} \\
& +\{0.518,-0.0148-0.141 i, 0.043+0.295 i, 0.382+0.571 i,-0.879+0.201 i,-0.128+0.442 i\} \epsilon .
\end{aligned}
$$

We fix the gauge $z_{1}=1, z_{2}=2, z_{3}=3$. There are two singular-I solutions, two singular-II solutions and two regular solutions:

$$
\begin{aligned}
& \operatorname{Regular}(1)\left\{\begin{array}{l}
z_{4} \rightarrow(1.925-0.124 i)+(0.0527+0.0364 i) \epsilon+(0.330-0.043 i) \epsilon^{2} \\
z_{+} \rightarrow(2.079-0.567 i)+(0.654+0.288 i) \epsilon+(1.460+1.530 i) \epsilon^{2} \\
z_{-} \rightarrow(1.549+0.129 i)-(0.590-0.787 i) \epsilon+(0.296+1.238 i) \epsilon^{2}
\end{array}\right. \\
& \operatorname{Regular}(2)\left\{\begin{array}{l}
z_{4} \rightarrow(1.996+0.0401 i)+(0.0904+0.0436 i) \epsilon+(0.141-0.512 i) \epsilon^{2} \\
z_{+} \rightarrow(2.049+0.132 i)+(0.0966-0.0383 i) \epsilon+(-0.346-1.292 i) \epsilon^{2} \\
z_{-} \rightarrow(1.590-0.286 i)-(0.123-2.415 i) \epsilon+(9.723-7.520 i) \epsilon^{2}
\end{array}\right. \\
& \text { Singular I }(1)\left\{\begin{array}{l}
z_{4} \rightarrow 1.911-(0.267-0.265 i) \epsilon+(-0.599+1.144 i) \epsilon^{2} \\
z_{+} \rightarrow(1.726-0.479 i)+(0.112-1.692 i) \epsilon+(-0.604-4.715 i) \epsilon^{2} \\
z_{-} \rightarrow(1.726-0.479 i)-(0.112-1.692 i) \epsilon+(0.604+4.715 i) \epsilon^{2}
\end{array}\right. \\
& \text { Singular I }(2)\left\{\begin{array}{l}
z_{4} \rightarrow 1.911+(0.152+0.0200 i) \epsilon+(0.0931-0.514 i) \epsilon^{2} \\
z_{+} \rightarrow(1.785+0.232 i)+(0.158-0.509 i) \epsilon+(-1.055-0.466 i) \epsilon^{2} \\
z_{-} \rightarrow(1.785+0.232 i)-(0.158-0.509 i) \epsilon+(1.055+0.466 i) \epsilon^{2}
\end{array}\right. \\
& \text { Singular II }(1)\left\{\begin{aligned}
z_{4} & \rightarrow 1.911-(0.130-0.336 i) \epsilon+(0.359+1.232 i) \epsilon^{2} \\
z_{+} & \rightarrow(1.958-0.123 i)+(0.285-0.0967 i) \epsilon-(0.0694+0.129 i) \epsilon^{2} \\
z_{-} & \rightarrow(1.958-0.123 i)+(0.285-0.0967 i) \epsilon+(0.0694+0.129 i) \epsilon^{2}
\end{aligned}\right. \\
& \text { Singular II }(2)\left\{\begin{array}{l}
z_{4} \rightarrow 1.911+(0.0151-0.0503 i) \epsilon+(0.231-0.189 i) \epsilon^{2} \\
z_{+} \rightarrow(2.064+0.345 i)+(0.0666+1.0254 i) \epsilon-(0.223-0.747 i) \epsilon^{2} \\
z_{-} \rightarrow(2.064+0.345 i)+(0.0666+1.0254 i) \epsilon+(0.223-0.747 i) \epsilon^{2} .
\end{array}\right.
\end{aligned}
$$

\footnotetext{
${ }^{11}$ In [52] it has been shown that although singular I solutions give nonzero contributions at the integrand level, it can be removed for some theories since the one-loop CHY-integrand is defined up to terms integrated to zero.
} 
TABLE XIX. Contribution of two singular solutions and regular respectively.

\begin{tabular}{lccccc}
\hline \hline & $z_{1}-z_{2}$ & Measure & $I_{\mathrm{CHY}} \sim \frac{1}{\left(z_{1}-z_{2}\right)^{2}}$ & $I_{\mathrm{CHY}} \sim \frac{1}{\left(z_{1}-z_{2}\right)^{1}}$ & $I_{\mathrm{CHY}} \sim \frac{1}{\left(z_{1}-z_{2}\right)^{0}}$ \\
\hline Singular-I & $\epsilon$ & 1 & $\epsilon^{-2}$ & $\epsilon^{-1}$ & 1 \\
Singular-II & $\epsilon^{2}$ & $\epsilon$ & $\epsilon^{-4}$ & $\epsilon^{-2}$ & 1 \\
Regular & 1 & 1 & 1 & 1 & 1 \\
Contribution of Singular-I & & & $\epsilon^{-2}$ & $\epsilon^{-1}$ & 1 \\
Contribution of Singular-II & 1 & 1 & $\epsilon^{-3}$ & 1 & $\epsilon$ \\
Contribution of Regular & 1 & 1 & & 1 \\
\hline \hline
\end{tabular}

TABLE XX. The measure part of each solution in forward limit.

\begin{tabular}{lc}
\hline \hline Solution & Measure \\
\hline Regular (1) & $0.201709+0.315957 i+O(\epsilon)$ \\
Regular (2) & $0.00828429+0.000758566 i+O(\epsilon)$ \\
SingularI (1) & $0.09663-0.37947 i+O(\epsilon)$ \\
SingularI (2) & $-0.0222706-0.0106388 i+O(\epsilon)$ \\
SingularII (1) & $(-0.000290196+0.0000925484 i) \epsilon+O\left(\epsilon^{2}\right)$ \\
SingularII (2) & $(0.606991+0.0403871 i) \epsilon+O\left(\epsilon^{2}\right)$ \\
\hline \hline
\end{tabular}

The measure part of each solution is in Table XX. which agree with our computation (the third column of Table XIX). Next we choose three different CHY-integrands with pole index $\chi\left[S_{+-}\right]=0,-1,-2$ respectively to show the behavior of Table XIX:

(i) $I_{1}=P T(1234+-) P T(1342-+)$ with $\chi\left[S_{+-}\right]=0$ : For this example (see Table XXI), there are poles $S_{+-} \sim \epsilon^{-2}$ and $S_{1+-} \sim \epsilon^{-1}$, so the leading contribution comes only from Singular II solutions.

(ii) $I_{2}=P T(1234+-) P T(1+234-)$ with $\chi\left[S_{+-}\right]=-1$ : For this example (see Table XXII), there is pole $S_{1+-} \sim \epsilon^{-1}$, so the leading contribution comes from both Singular I and Singular II solutions.

(iii) $I_{3}=P T(123+4-) P T(1+423-)$ with $\chi\left[S_{+-}\right]=$ -2: For this example (see Table XXIII), there is no singular pole, so the leading contribution comes from regular solutions as well as Singular I solutions.

TABLE XXI. The contribution of each solution of CHYintegrand $P T(1234+-) P T(1342-+)$.

\begin{tabular}{lc}
\hline \hline Solution & Integration \\
\hline Regular (1) & $-(9.3103+1.98347 i)$ \\
Regular (2) & $(2.81938-6.61778 i)$ \\
SingularI (1) & $-\frac{1}{\epsilon^{2}}(0.622372+0.426007 i)$ \\
SingularI (2) & $-\frac{1}{\epsilon^{2}}(0.726396+1.70513 i)$ \\
SingularII (1) & $\frac{1}{\epsilon^{3}}(0.657355+0.819951 i)$ \\
SingularII (2) & $-\frac{1}{\epsilon^{3}}(0.862234-0.0145693 i)$ \\
Summation & $-\frac{1}{\epsilon^{3}}(0.204879-0.83452 i)$ \\
Amplitude & $-\frac{1}{S_{34} S_{+-} S_{1+-}}$ \\
Numerical amplitude & $-\frac{1}{\epsilon^{3}}(0.204879-0.83452 i)$ \\
\hline \hline
\end{tabular}

\section{B. Applications in one-loop CHY-Integrands}

One main motivation of our current study is to understand that under the forward limit, what is the contributions of singular solutions for one-loop CHY-integrands constructed

TABLE XXII. The contribution of each solution of CHYintegrand $P T(1234+-) P T(1+234-)$.

\begin{tabular}{lc}
\hline \hline Solution & Integration \\
\hline Regular (1) & $-(2.00263-7.49773 i)$ \\
Regular (2) & $(3.43278+1.47441 i)$ \\
Singular I (1) & $-\frac{1}{\epsilon}(0.0475165-1.0134 i)$ \\
Singular I (2) & $-\frac{1}{\epsilon}(1.25809+1.02357 i)$ \\
Singular II (1) & $-\frac{1}{\epsilon}(0.423532+0.0715691 i)$ \\
Singular II (2) & $-\frac{1}{\epsilon}(0.524593+0.208376 i)$ \\
Summation & $\frac{1}{\epsilon}(1.20454-0.126638 i)$ \\
Amplitude & $\frac{1}{S_{1-} S_{23} S_{1+-}}+\frac{1}{S_{1-} S_{34} S_{1+-}}$ \\
Numerical amplitude & $\frac{1}{\epsilon}(1.20454-0.126638 i)$ \\
\hline \hline
\end{tabular}

TABLE XXIII. The contribution of each solution of CHYintegrand $P T(123+4-) P T(1+423-)$.

\begin{tabular}{lc}
\hline \hline Solution & Integration \\
\hline Regular (1) & $(36.3435-21.9682 i)$ \\
Regular (2) & $-(49.0302-26.6536 i)$ \\
Singular I (1) & $(19.3907-18.34 i)$ \\
Singular I (2) & $-(18.0838+0.689813 i)$ \\
Singular II (1) & $(1.43509+0.475868 i) \epsilon$ \\
Singular II (2) & $-(10.9922+0.404811 i) \epsilon$ \\
Summation & $-(11.3798+14.3444 i)$ \\
Amplitude & $\frac{1}{S_{1-} S_{23} S_{4+}}$ \\
Numerical amplitude & $-(11.3798+14.3444 i)$ \\
\hline \hline
\end{tabular}


using the forward limit method. In this subsection, we will use two types of one-loop CHY-integrands of the bi-adjoint scalar theory to provide some general picture, especially the different patterns of cancellation of singularities.

\section{The first type of integrands}

In [52] the one-loop CHY-integrand is given by

$$
m_{n}^{1-\text { loop }}[\pi \mid \rho]=\int \frac{d^{D} l}{(2 \pi)^{D}} \frac{1}{l^{2}} \lim _{k_{ \pm} \rightarrow \pm l} \sum_{\substack{\alpha \in c y c(\pi) \\ \beta \in y y(\rho)}} m_{n+2}^{\text {tree }}[-\alpha+\mid-\beta+]
$$

where $\alpha$ and $\beta$ are two PT factors and

$$
m_{n+2}^{\mathrm{tree}}[-\alpha+\mid-\beta+]=\int d \Omega_{\mathrm{CHY}} P T(-, \alpha(1), \ldots, \alpha(n),+) P T(-, \beta(1), \ldots, \beta(n),+)
$$

is the tree-level amplitudes of $(n+2)$ particles defined by the CHY-integrand of two PT factors.

To have a concrete picture, let us consider a simple example, i.e., one loop integrands $m_{4}^{1-\text { loop }}$ [1234|1243]. From (5.9), this loop integrand is given by the sum of all possible combinations of PT-factor sets $\{\mathrm{PT}(1+-234), \mathrm{PT}(12+$ -34), $\mathrm{PT}(123+-4), \mathrm{PT}(1234+-)\}$ and $\{\mathrm{PT}(1+-243), \mathrm{PT}(12+-43), \mathrm{PT}(124+-3), \mathrm{PT}(1243+-)\}$. As in the previous section, a set of momentum with forward limit parameter $\epsilon$ has been taken as following:

$$
\begin{aligned}
k_{1} \rightarrow & \{1.04,0.113,-0.156,0.775,0.667,0\} \\
k_{2} \rightarrow & \{1.43,-0.913,-0.771,-0.727,-0.295,0\} \\
k_{3} \rightarrow & \{-0.931,0.383,-0.508,-0.344,-0.587,0\}+\{-0.699 \epsilon,(0.4+0.304 i) \epsilon,(-0.529+0.23 i) \epsilon, 0.0745 \epsilon,-0.434 \epsilon, 0\} \\
k_{4} \rightarrow & \{-1.54,0.417,1.43,0.296,0.215,0\}+\{-1.15 \epsilon,(0.354-0.641 i) \epsilon,(1.22+0.186 i) \epsilon,-0.191 \epsilon,-0.342 \epsilon, 0\} \\
k_{+} \rightarrow & \{0.45,-1.02-0.34 i,-0.548,0.287-1.21 i,-0.597,0\} \\
& +\{0.923 \epsilon,(-0.377+0.168 i) \epsilon,(-0.344-0.208 i) \epsilon, 0.0584 \epsilon, 0.388 \epsilon,(0.714-0.0115 i) \epsilon\} \\
k_{-} \rightarrow & \{-0.45,1.02+0.34 i, 0.548,-0.287+1.21 i, 0.597,0\} \\
& +\{0.923 \epsilon,(-0.377+0.168 i) \epsilon,(-0.344-0.208 i) \epsilon, 0.0584 \epsilon, 0.388 \epsilon,(-0.714+0.0115 i) \epsilon\}
\end{aligned}
$$

For current example, there are $(n-3) !=6$ solutions. Under the forward limit, they are divided into three classes according to the asymptotic behavior, i.e., regular solutions, singular I and singular II solutions, and each of them contains two solutions:

$$
\begin{gathered}
\text { Regular (1) }\left\{\begin{array}{l}
z_{4} \rightarrow(1.99+0.074 i)-(0.21-0.0669 i) \epsilon+(0.0316-0.313 i) \epsilon^{2} \\
z_{+} \rightarrow(2.3+0.135 i)-(0.283-0.543 i) \epsilon-(1.36+1.09 i) \epsilon^{2} \\
z_{-} \rightarrow(2.01+0.175 i)-(0.0946-0.132 i) \epsilon+(0.0981-0.0337 i) \epsilon^{2}
\end{array}\right. \\
\text { Regular (2) }\left\{\begin{array}{l}
z_{4} \rightarrow(2.04-0.483 i)-(0.496+0.172 i) \epsilon-(0.182-1.33 i) \epsilon^{2} \\
z_{+} \rightarrow(1.78-0.594 i)-(0.234-0.198 i) \epsilon+(0.678+1.02 i) \epsilon^{2} \\
z_{-} \rightarrow(2.52+0.273 i)+(0.516+0.15 i) \epsilon+(0.354-0.104 i) \epsilon^{2}
\end{array}\right. \\
\text { Singular I(1) }\left\{\begin{array}{l}
z_{4} \rightarrow 1.89-(0.0953+0.0598 i) \epsilon-(0.469+0.562 i) \epsilon^{2} \\
z_{+} \rightarrow(1.82+0.0335 i)+(0.00702-0.103 i) \epsilon+(0.207-0.0868 i) \epsilon^{2} \\
z_{-} \rightarrow(1.82+0.0335 i)-(0.00702-0.103 i) \epsilon-(0.207-0.0868 i) \epsilon^{2}
\end{array}\right. \\
\text { Singular I (2) }\left\{\begin{array}{l}
z_{4} \rightarrow 1.89-(0.0845+0.0676 i) \epsilon-(0.0545+0.0212 i) \epsilon^{2} \\
z_{+} \rightarrow(2.39+0.184 i)-(0.344-0.0399 i) \epsilon+(0.176-0.0308 i) \epsilon^{2} \\
z_{-} \rightarrow(2.39+0.184 i)+(0.344-0.0399 i) \epsilon-(0.176-0.0308 i) \epsilon^{2}
\end{array}\right.
\end{gathered}
$$




$$
\begin{gathered}
\text { Singular II }(1)\left\{\begin{array}{l}
z_{4} \rightarrow 1.89-(0.0923+0.236 i) \epsilon+(0.317+0.0928 i) \epsilon^{2} \\
z_{+} \rightarrow(1.89-0.376 i)+(0.103+0.191 i) \epsilon-(6.61-1.44 i) \epsilon^{2} \\
z_{-} \rightarrow(1.89-0.376 i)+(0.103+0.191 i) \epsilon-(6.37-1.27 i) \epsilon^{2}
\end{array}\right. \\
\text { Singular II }(2)\left\{\begin{array}{l}
z_{4} \rightarrow 1.89-(0.0874-0.109 i) \epsilon-(0.00897-0.265 i) \epsilon^{2} \\
z_{+} \rightarrow(1.97+0.196 i)+(0.0645+0.0918 i) \epsilon-(2.57-1.42 i) \epsilon^{2} \\
z_{-} \rightarrow(1.97+0.196 i)+(0.0645+0.0918 i) \epsilon-(2.64-1.18 i) \epsilon^{2}
\end{array}\right.
\end{gathered}
$$

For the numerical evaluation, we will take two different approaches. In the first approach, for each term in the 16 combinations of CHY-integrand (5.9), we sum over 6 solutions first, and the result is given in the Table XXIV.

In the Table XXIV, although most of the terms will have leading divergence $\frac{1}{\epsilon^{3}}$, when summing over 16 terms, all divergences will cancel each other, and we are left with finite results under the forward limit as expected.

Although the divergence is not shown at the final, how the cancellation happens is not clear. To better understand, we take the second approach: for each solution, we put it to all 16 terms and sum them up first, and then sum up six solutions. One very interesting observation is that the divergence will cancel each other after summing 16 terms for each solution as shown in Table XXV. We could also verify this matter by summing 16 terms analytically, which is given by:

$$
\begin{aligned}
& N=\left(z_{1+} z_{2-}\left(z_{13} z_{2+} z_{3-} z_{4+} z_{4-}+z_{1-} z_{3+}\left(z_{2+} z_{34} z_{4-}-z_{24} z_{3-} z_{4+}\right)\right)-z_{12} z_{1-} z_{2+} z_{3+} z_{3-} z_{4+} z_{4-}\right) \\
& \times\left(z_{12} z_{1-} z_{2+} z_{3+} z_{3-} z_{4+} z_{4-}+z_{1+} z_{2-}\left(z_{1-} z_{4+}\left(z_{2+} z_{34} z_{3-}+z_{23} z_{3+} z_{4-}\right)-z_{14} z_{2+} z_{3+} z_{3-} z_{4-}\right)\right) \\
& I=\frac{N}{z_{12}^{2} z_{13} z_{14} z_{1+}^{2} z_{1-}^{2} z_{23} z_{24} z_{2+}^{2} z_{2-}^{2} z_{34}^{2} z_{3+}^{2} z_{3-}^{2} z_{4+}^{2} z_{4-}^{2} z_{+-}^{2}}
\end{aligned}
$$

As one can check, for each solution, the $N$ will give $\epsilon^{a}$ factor to cancel the singular from the denominator and the measure.

The cancelation pattern observed above for the integrand (5.9) is not a coincident. In fact, in the construction of the

TABLE XXIV. Leading order of the tree integrand.

\begin{tabular}{lcc}
\hline \hline Integrand & $\begin{array}{c}\text { Asymptotic level } \\
\text { of integrand }\end{array}$ & $\begin{array}{c}\text { Leading } \\
\text { order }\end{array}$ \\
\hline$P T(1+-234) P T(1+-243)$ & $\frac{1}{z_{+-}^{2}}$ & $\frac{0.074+0.006 i}{\epsilon^{3}}$ \\
$P T(1+-234) P T(12+-43)$ & $\frac{1}{z_{+-}^{2}}$ & $-\frac{0.032+0.0013 i}{\epsilon^{3}}$ \\
$P T(1+-234) P T(124+-3)$ & $\frac{1}{z_{+-}^{2}}$ & 0 \\
$P T(1+-234) P T(1243+-)$ & $\frac{1}{z_{+-}^{2}}$ & $-\frac{0.042+0.0047 i}{\epsilon^{3}}$ \\
$P T(12+-34) P T(1+-243)$ & $\frac{1}{z_{+-}^{2}}$ & $-\frac{0.032+0.0013 i}{\epsilon^{3}}$ \\
$P T(12+-34) P T(12+-43)$ & $\frac{1}{z_{+-}^{2}}$ & $\frac{0.032+0.0013 i}{\epsilon^{3}}$ \\
$P T(12+-34) P T(124+-3)$ & $\frac{1}{z_{+-}^{2}}$ & $-\frac{0.04-0.0093 i}{\epsilon^{3}}$ \\
$P T(12+-34) P T(1243+-)$ & $\frac{1}{z_{+-}^{2}}$ & $\frac{0.04-0.0093 i}{\epsilon^{3}}$ \\
$P T(123+-4) P T(1+-243)$ & $\frac{1}{z_{+-}^{2}}$ & 0 \\
$P T(123+-4) P T(12+-43)$ & $\frac{1}{z_{+-}^{2}}$ & $-\frac{0.029+0.0087 i}{\epsilon^{3}}$ \\
$P T(123+-4) P T(124+-3)$ & $\frac{1}{z_{+-}^{2}}$ & $\frac{-0.072-0.00057 i}{\epsilon^{3}}$ \\
$P T(123+-4) P T(1243+-)$ & $\frac{1}{z_{+-}^{2}}$ & $-\frac{0.04-0.0093 i}{\epsilon^{3}}$ \\
$P T(1234+-) P T(1+-243)$ & $\frac{1}{z_{+-}^{2}}$ & $-\frac{0.042+0.0047 i}{\epsilon^{3}}$ \\
$P T(1234+-) P T(12+-43)$ & $\frac{1}{z_{+-}^{2}}$ & $\frac{0.029+0.0087 i}{\epsilon^{3}}$ \\
$P T(1234+-) P T(124+-3)$ & $\frac{1}{z_{+-}^{2}}$ & $-\frac{0.029+0.0087 i}{\epsilon^{3}}$ \\
$P T(1234+-) P T(1243+-)$ & $\frac{1}{z_{+-}^{2}}$ & $\frac{0.042+0.0047 i}{\epsilon^{3}}$ \\
\hline \hline
\end{tabular}

CHY-integrand, the tadpole contribution under the forward limit is manifest avoid, while the massless bubble must be canceled by different terms in the combinations.

\section{The second type of integrands}

A different idea to remove the tadpole and massless bubble singularities has been used in [54]. Based on this idea, different one-loop CHY-integrand has been constructed by multiplying each term with the proper polepicking operators. For the example $m_{4}^{1-\text { loop }}$ [1234|1243], there are only three terms as given in the Table XXVI. By the construction, for each term, the cancellation of divergence comes from the summing over six solutions as shown in the second column of the Table XXVI. The front of the PT integrand is the pick-pole factor, which is used to pick out the divergence part refer to $[47,55]$. More accurately, the cancellation happens when and only when summing

TABLE XXV. Leading order of the loop integrand.

\begin{tabular}{lc}
\hline \hline Solution & Leading order \\
\hline Regular (1) & $(0.0738-0.128 i)+O(\epsilon)$ \\
Regular (2) & $-(0.126+0.00619 i)+O(\epsilon)$ \\
Singular I (1) & $(0.0277+0.2 i)+O(\epsilon)$ \\
Singular I (2) & $-(0.055-0.214 i)+O(\epsilon)$ \\
Singular II (1) & $(0.00452+0.00782 i) \epsilon+O\left(\epsilon^{2}\right)$ \\
Singular II (2) & $(0.0145+0.0407 i) \epsilon+O\left(\epsilon^{2}\right)$ \\
Total & $-(0.079-0.28 i)+O(\epsilon)$ \\
\hline \hline
\end{tabular}


TABLE XXVI. Leading order of the tree integrand.

\begin{tabular}{ll}
\hline \hline Integrand & Leading order \\
\hline$\left(1-\frac{z_{14}^{2} z_{2-} z_{-+}}{z_{12} z_{1-} z_{4-} z_{4+}}-\frac{z_{23}^{2} z_{1+} z_{-+}}{z_{12} z_{3-} z_{2+} z_{3+}}-\frac{z_{2-} z_{1+}}{z_{1-} z_{2+}}\right) P T(1+-234) P T(1+-243)$ & $0.015+0.03 i$ \\
$\left(1-\frac{z_{12}^{2} z_{3-} z_{++}}{z_{22} z_{1-} z_{2-} z_{1+}}-\frac{z_{3-} z_{2+}}{z_{2-} z_{3+}}\right) P T(12+-34) P T(12+-43)$ & $0.00063+0.0163 i$ \\
$\left(1-\frac{z_{12}^{2} z_{4+} z_{-+}}{z_{14} z_{2-} z_{1+} z_{2+}}-\frac{z_{1-} z_{4+}}{z_{4-} z_{1+}}\right) P T(1234+-) P T(1243+-)$ & $0.00087+0.0173 i$ \\
\hline \hline
\end{tabular}

over singular I and singular II solutions. Just summing over singular I solutions or singular II solutions, the divergence will not be cancelled.

\section{CONCLUSION}

Motivated by our curiosity of the singular solutions and potential application to other frontiers of researches, for example, the construction of two-loop CHY-integrands by double forward limits, in this paper, we have initiated the systematic study of the relation between the singular kinematic configurations and the singular solutions of scattering equations. We find that the singular solutions will always lead to singular kinematics. Furthermore, the layer structure of singular solutions gives a clear picture of the structure of singular kinematics. However, the reverse is not always transparent, as shown by the soft limit and forward limit. From these examples, we guess the compatibility of various singular poles can characterize the singular kinematics. Although we can not give rigorous proof, from the examples discussed in this paper, it seems that compatible singular poles will lead to singular solutions, but noncompatible will not give singular solutions.

Although we have discussed several types of singularities in this paper, it is obvious that there are many other types of singularities. One-by-one analysis can be done using the method presented in the paper. However, it is more desirable to have a systematical algorithm so that the relation between the singular kinematic configurations and the singular solutions of scattering equations can be well established.

\section{ACKNOWLEDGMENTS}

We would like to thank Ye Yuan and Song He for very useful discussion. This work is supported by Qiu-Shi Funding and Chinese NSF funding under Grant No. 11935013, No. 11947301, No. 12047502 (Peng Huanwu Center).

\section{APPENDIX: KINEMATICS}

In this note, we focus on solutions of scattering equations defined by (1.3) or the equivalent polynomial equations given in [13]

$$
0=h_{m} \equiv \sum_{S \in A,|S|=m} k_{S}^{2} z_{S}, \quad 2 \leq m \leq n-2,
$$

Where the sum is over all $\frac{n !}{(n-m) ! m !}$ subsets $S$ of $A=$ $\{1,2, \ldots, n\}$ with exactly $m$ elements and $k_{S}=\sum_{b \in S} k_{b}$ and $z_{S}=\prod_{b \in S} z_{b}$. It is worth pointing out that there are exactly $(n-3)$ independent equations of the form (A1), since the cases of $m=1, n-1, n$ are trivially true by momentum conservation plus the on-shell conditions. For general kinematics, it is impossible to write down analytic results for $n \geq 6$, thus to study the properties of solutions, we need to rely on the numerical method. One important step of the numerical calculation is the parametrization of kinematics, especially when we try to study different limit processes, such as the factorization limit, the forward limit and the soft limit, etc.

There are two ways to define a kinematic configuration. The first way is to use the Lorentz invariant combination $k_{i} \cdot k_{j}$. With momentum conservation and null conditions, there are $\frac{n(n-3)}{2}$ independent contractions. ${ }^{12}$ To see it, using momentum conservation, we can eliminate $p_{n}$. For remaining $(n-1)$ momenta, there are $\left(\begin{array}{c}n-1 \\ 2\end{array}\right)=\frac{(n-1)(n-2)}{2}$ contractions. A further constraint comes from

$$
0=k_{n}^{2}=\left(\sum_{i=1}^{n} k_{i}\right)^{2}=\sum_{i<j} 2 k_{i} \cdot k_{j}
$$

thus we get $\frac{(n-1)(n-2)}{2}-1=\frac{n(n-3)}{2}$ contractions. Let us apply this method to the kinematic configuration of forward limit of $(n+2)$ legs defined by

$$
L_{+}+L_{-}=2 \epsilon q, \quad L_{+} \cdot L_{-}=2 \epsilon^{2} q^{2}, \quad q^{2} \neq 0
$$

where $t=0$ is the forward limit. For general $t \neq 0$, we take following data to parameterize kinematics:

$$
\begin{gathered}
q^{2} ; \quad P_{i} \cdot P_{j}, 1 \leq i<j \leq n-1 ; \quad L_{+} \cdot P_{i}, q \cdot P_{i}, \\
\quad i=1, \ldots, n-1
\end{gathered}
$$

There are $\frac{(n-1)(n-2)}{2}+1+2(n-1)=\frac{(n+2)(n-1)}{2}+1$ combinations, thus they are not independent. One extra constraint comes from the on-shell condition $P_{n}^{2}=0$, i.e.,

\footnotetext{
${ }^{12}$ To get this counting, we have neglected the constraints coming from the dimension of space-time.
} 


$$
\begin{aligned}
0 & =\frac{1}{2}\left(\sum_{i=1}^{n-1} P_{i}+2 \epsilon q\right)^{2} \\
& =\sum_{1 \leq i<j \leq n-1} P_{i} \cdot P_{j}+2 \epsilon q \cdot \sum_{i=1}^{n-1} P_{i}+2 \epsilon^{2} q^{2}
\end{aligned}
$$

Using above data, other Lorentz contractions are given by

$$
\begin{aligned}
& L_{-} \cdot P_{i}=-L_{+} \cdot P_{i}+2 \epsilon q \cdot P_{i} ; \\
& P_{i} \cdot P_{n}=-2 \epsilon q \cdot P_{i}-\sum_{j=1, j \neq i}^{n-1} P_{i} \cdot P_{j} \\
& L_{+} \cdot P_{n}=-2 \epsilon^{2} q^{2}-\sum_{j=1}^{n-1} L_{+} \cdot P_{j} ; \\
& L_{-} \cdot P_{n}=-2 \epsilon^{2} q^{2}+\sum_{j=1}^{n-1}\left(L_{+} \cdot P_{j}-2 \epsilon q \cdot P_{j}\right)
\end{aligned}
$$

By momentum conservation. With the above choice, we need to determine how data in (A4) depend on $\epsilon$ to describe the limit process. This is not a trivial task. For example, a naive choice is to take $q^{2}, L_{+} \cdot P_{i}, q \cdot P_{i}$ as well as all $P_{i} \cdot P_{j}$ except one by constraint (A5) as invariant data under the limit. This naive choice will have the maximum number of combinations, which are independent of $\epsilon$. However, this choice is wrong because now we will have $L_{+}$to be invariant under the forward limit, thus

$$
\begin{aligned}
L_{-}^{2} & =\left(-L_{+}+2 \epsilon q\right)^{2}=L_{+}^{2}-4 \epsilon L_{+} \cdot q+4 \epsilon^{2} q^{2} \\
& =-4 \epsilon L_{+} \cdot q+4 \epsilon^{2} q^{2}
\end{aligned}
$$

can not be zero for all value of $\epsilon$. The lesson from this example is the following. Although taking Lorentz invariant combinations to be the independent input data for the kinematics looks simple, it is not easy to determine how they depend on the parameter $\epsilon$, which prescribes the limit process.

To avoid the above-mentioned subtlety, we take the second parametrization method, i.e., parametrising directly in the momentum component form. The first step to set up a general kinematic configuration is that there is no subset of $A$ such that $S_{A}=0$. Starting from this choice, we can make various limit procedure:

(i) The factorization limit: For this case, we consider several situations. The first situation is there is one and only one $S_{A} \rightarrow 0$. To reach this goal, a simple way is to use BCFW-deformation, i.e., while keeping all other $p_{i}$ 's invariant, we deform

$$
\begin{aligned}
p_{1}(z) & =p_{1}+z q, \quad p_{n}(z)=p_{n}-z q, \\
q^{2} & =q \cdot p_{1}=q \cdot p_{n}=0
\end{aligned}
$$

For any subset $1 \in A, n \notin A$, it is easy to know that when

$$
z_{A}=-\frac{P_{A}^{2}}{2 P_{A} \cdot q} \Rightarrow S_{A}\left(z_{A}\right)=0
$$

Now we parametrize the kinematic as

$$
\begin{aligned}
p_{1}(\epsilon) & \equiv p_{1}+z_{A} q+\epsilon q, \quad p_{n}(\epsilon)=p_{n}-z_{A} q-\epsilon q, \\
q^{2} & =q \cdot p_{1}=q \cdot p_{n}=0 \quad(\mathrm{~A} 10)
\end{aligned}
$$

so when $\epsilon \rightarrow 0$ the factorization limit has been reached. A similar idea can be used to putting several $S_{A} \rightarrow 0$ at the same time. For example, using two different pairs of BCFW deformations

$$
\begin{aligned}
p_{1}\left(w_{1}\right) & =p_{1}+w_{1} q_{1 n}, \quad p_{n}\left(w_{1}\right)=p_{n}-w_{1} q_{1 n}, \\
q_{1 n}^{2} & =q_{1 n} \cdot p_{1}=q_{1 n} \cdot p_{n}=0 \\
p_{2}\left(w_{2}\right) & =p_{2}+w_{2} q_{23}, \quad p_{3}\left(w_{2}\right)=p_{3}-w_{2} q_{23}, \\
q_{23}^{2} & =q_{23} \cdot p_{1}=q_{23} \cdot p_{3}=0
\end{aligned}
$$

we can set two poles $S_{A}, S_{B} \rightarrow 0$ with $1 \in A, n \notin A$ and $2 \in B, 3 \notin B$ at the same time. We want to emphasize that the poles $S_{A}, S_{B}$ can be compatible or not compatible. Different patterns will give different behaviors of singular solutions. A special variation of two BCFW-deformations is that they share one common node, for example, $p_{1}$, i.e.,

$$
\begin{aligned}
p_{1}\left(w_{1}\right) & =p_{1}+w_{1} q_{1 n}+w_{2} q_{12}, \\
p_{n}\left(w_{1}\right) & =p_{n}-w_{1} q_{1 n}, \quad p_{2}\left(w_{2}\right)=p_{2}-w_{2} q_{12}, \\
q_{1 n}^{2} & =q_{1 n} \cdot p_{1}=q_{1 n} \cdot p_{n}=0, \\
q_{12}^{2} & =q_{12} \cdot p_{1}=q_{12} \cdot p_{2}=0 \quad q_{1 n} \cdot q_{12}=0,
\end{aligned}
$$

(ii) The forward limit: For the pair of the forward limit, we take them as

$$
\begin{aligned}
L_{+} & =(L+\epsilon q, \mu), \quad L_{-}=(-L+\epsilon q,-\mu) \\
q^{2}, L^{2} & \neq 0,
\end{aligned}
$$

where we have lifted $L_{ \pm}$to be null in higher dimension. To make sure the null condition for any value of $\epsilon$, we need to impose conditions

$$
q \cdot L=0, \quad-\mu^{2}=L^{2}+\epsilon^{2} q^{2}
$$

For other $n$ momenta, we find $n$ pairs of $p_{i}, q_{i}$, $i=1, \ldots, n$ such that 


$$
p_{i}^{2}=q_{i}^{2}=p_{i} \cdot q_{i}=0, \quad i=1, \ldots, n ; \quad \sum_{i=1}^{n} p_{i}=0, \quad \sum_{i=1}^{n} q_{i}=2 q
$$

and use them to define

$$
P_{i}=p_{i}-\epsilon q_{i}
$$

Above choice of the forward limit is very general. We can make a simple choice with the minimum numbers of $P_{i}$ depending on $\epsilon$. The procedure is the following:

(a1) First setting $q_{j}=0, j=1, \ldots, n-2$, so $P_{j}=p_{j}$.

(a2) Taking a null momentum $k_{n-1}$, we construct

$$
p_{n-1}=-\frac{K^{2}}{2 K \cdot k_{n-1}} k_{n-1}, \quad p_{n}=-K+\frac{K^{2}}{2 K \cdot k_{n-1}} k_{n-1}, \quad K=\sum_{j=1}^{n-2} p_{j}
$$

(a3) Taking null momenta $q_{n-1}, q_{n}$ such that $p_{a} \cdot q_{a}=0, a=n-1, n$ and $q_{n} \cdot q_{n-1} \neq 0$ and choosing $b_{n-1}, \beta_{n}$ such that $L \cdot\left(\beta_{n-1} q_{n-1}+\beta_{n} q_{n}\right)=0$, we can write down

$$
P_{n-1}=p_{n-1}-2 \epsilon \beta_{n-1} q_{n-1}, \quad P_{n}=p_{n}-2 \epsilon \beta_{n} q_{n}, \quad q=\beta_{n-1} q_{n-1}+\beta_{n} q_{n},
$$

Above construction (A18) and (A19) have two exceptions. The first case is $K=0$, i.e., the case $n=2$. For this one, we can trivially take $p_{n}=-p_{n-1}$ in (A18). The second case is that $K \neq 0$, but $K^{2}=0$, which will happen when $n=3$ (for general momentum configuration, it could not be true for $n \geq 4$ ). For this one, we can take

$$
\begin{array}{ll}
P_{1}=-p_{2}-p_{3}, & P_{2}=p_{2}-2 \epsilon q_{2}, \quad P_{3}=p_{3}-2 \epsilon q_{3}, \\
L_{+}=(L+\epsilon q, \mu), & L_{-}=(-L+\epsilon q,-\mu), \quad q=q_{2}+q_{3}, \quad \mu^{2}=L^{2}+\epsilon^{2} q^{2}
\end{array}
$$

with conditions

$$
\begin{aligned}
p_{i}^{2} & =q_{i}^{2}=0=p_{3} \cdot p_{2}=L \cdot q, \quad i=2,3 \\
q_{2} \cdot p_{2} & =0=q_{3} \cdot p_{3}, \quad q_{2} \cdot q_{3}, q_{2} \cdot p_{3}, q_{3} \cdot p_{2} \neq 0
\end{aligned}
$$

(iii) The soft limit: The kinematic parametrization is following [56]. Assuming the $p_{s}$ is the soft particle, we take two arbitrary momenta, for example, $a, n$ and make the shifting

$$
\tilde{\lambda}_{a}(\epsilon)=\tilde{\lambda}_{a}+(1-\epsilon) \frac{\langle n \mid s\rangle}{\langle n \mid a\rangle} \tilde{\lambda}_{s}, \quad \tilde{\lambda}_{n}(\epsilon)=\tilde{\lambda}_{n}+(1-\epsilon) \frac{\langle a \mid s\rangle}{\langle a \mid n\rangle} \tilde{\lambda}_{s}, \quad \tilde{\lambda}_{s}(\epsilon)=\epsilon \tilde{\lambda}_{s}
$$

It is easy to see that

$$
\lambda_{a} \tilde{\lambda}_{a}+\lambda_{n} \tilde{\lambda}_{n}+\lambda_{s} \tilde{\lambda}_{s}=\lambda_{a} \tilde{\lambda}_{a}(\epsilon)+\lambda_{n} \tilde{\lambda}_{n} \lambda_{n}(\epsilon)+\lambda_{s} \tilde{\lambda}_{s}(\epsilon)
$$

and $\epsilon \rightarrow 0$ gives the soft limit. Although we have used the spinor formalism special for $D=4$, for general $D$-dimension, we can make the Lorenz transformation to put $p_{n}, p_{a}, p_{s}$ to the 4-dimensional subspace, thus above construction is general. 
[1] R. Britto, F. Cachazo, and B. Feng, New recursion relations for tree amplitudes of gluons, Nucl. Phys. B715, 499 (2005).

[2] R. Britto, F. Cachazo, B. Feng, and E. Witten, Direct Proof of Tree-Level Recursion Relation in Yang-Mills Theory, Phys. Rev. Lett. 94, 181602 (2005).

[3] Z. Bern, L. J. Dixon, D. C. Dunbar, and D. A. Kosower, Fusing gauge theory tree amplitudes into loop amplitudes, Nucl. Phys. B435, 59 (1995).

[4] Z. Bern, L. J. Dixon, D. C. Dunbar, and D. A. Kosower, One loop $n$ point gauge theory amplitudes, unitarity and collinear limits, Nucl. Phys. B425, 217 (1994).

[5] R. Britto, F. Cachazo, and B. Feng, Generalized unitarity and one-loop amplitudes in $N=4$ super-Yang-Mills, Nucl. Phys. B725, 275 (2005).

[6] F. Cachazo, S. He, and E. Y. Yuan, Scattering equations and Kawai-Lewellen-Tye orthogonality, Phys. Rev. D 90, 065001 (2014).

[7] F. Cachazo, S. He, and E. Y. Yuan, Scattering of Massless Particles in Arbitrary Dimensions, Phys. Rev. Lett. 113, 171601 (2014).

[8] F. Cachazo, S. He, and E. Y. Yuan, Scattering of massless particles: Scalars, gluons and gravitons, J. High Energy Phys. 07 (2014) 033.

[9] F. Cachazo, S. He, and E. Y. Yuan, Einstein-Yang-Mills scattering amplitudes from scattering equations, J. High Energy Phys. 01 (2015) 121.

[10] F. Cachazo, S. He, and E. Y. Yuan, Scattering equations and matrices: From Einstein to Yang-Mills, DBI and NLSM, J. High Energy Phys. 07 (2015) 149.

[11] C. Kalousios, Massless scattering at special kinematics as Jacobi polynomials, J. Phys. A 47, 215402 (2014).

[12] S. Weinzierl, On the solutions of the scattering equations, J. High Energy Phys. 04 (2014) 092.

[13] L. Dolan and P. Goddard, The polynomial form of the scattering equations, J. High Energy Phys. 07 (2014) 029.

[14] C. S. Lam, Permutation symmetry of the scattering equations, Phys. Rev. D 91, 045019 (2015).

[15] E. Casali and P. Tourkine, Infrared behaviour of the oneloop scattering equations and supergravity integrands, J. High Energy Phys. 04 (2015) 013.

[16] C. Kalousios, Scattering equations, generating functions and all massless five point tree amplitudes, J. High Energy Phys. 05 (2015) 054.

[17] F. Cachazo and H. Gomez, Computation of contour integrals on $\mathcal{M}_{0, n}$, J. High Energy Phys. 04 (2016) 108.

[18] R. Huang, J. Rao, B. Feng, and Y. H. He, An algebraic approach to the scattering equations, J. High Energy Phys. 12 (2015) 056.

[19] M. Søgaard and Y. Zhang, Scattering equations and global duality of residues, Phys. Rev. D 93, 105009 (2016).

[20] C. Cardona and C. Kalousios, Comments on the evaluation of massless scattering, J. High Energy Phys. 01 (2016) 178.

[21] C. Cardona and C. Kalousios, Elimination and recursions in the scattering equations, Phys. Lett. B 756, 180 (2016).

[22] L. Dolan and P. Goddard, General solution of the scattering equations, J. High Energy Phys. 10 (2016) 149.

[23] C. S. Lam and Y. P. Yao, Role of Möbius constants and scattering functions in Cachazo-He-Yuan scalar amplitudes, Phys. Rev. D 93, 105004 (2016).
[24] J. Bosma, M. Søgaard, and Y. Zhang, The polynomial form of the scattering equations is an $H$-basis, Phys. Rev. D 94, 041701 (2016).

[25] M. Zlotnikov, Polynomial reduction and evaluation of treeand loop-level CHY amplitudes, J. High Energy Phys. 08 (2016) 143.

[26] F. Cachazo, S. Mizera, and G. Zhang, Scattering equations: Real solutions and particles on a line, J. High Energy Phys. 03 (2017) 151.

[27] G. Chen, Y. K. E. Cheung, T. Wang, and F. Xu, A differential operator for integrating one-loop scattering equations, J. High Energy Phys. 01 (2017) 028.

[28] G. Chen, Y. K. E. Cheung, T. Wang, and F. Xu, A combinatoric shortcut to evaluate $\mathrm{CHY}$-forms, J. High Energy Phys. 06 (2017) 015.

[29] Z. Liu and X. Zhao, Bootstrapping solutions of scattering equations, J. High Energy Phys. 02 (2019) 071.

[30] G. De Laurentis, Analytical amplitudes from numerical solutions of the scattering equations, J. High Energy Phys. 02 (2020) 194.

[31] C. Baadsgaard, N. E. J. Bjerrum-Bohr, J. L. Bourjaily, and P. H. Damgaard, Integration rules for scattering equations, J. High Energy Phys. 09 (2015) 129.

[32] C. Baadsgaard, N. E. J. Bjerrum-Bohr, J. L. Bourjaily, and P. H. Damgaard, Scattering equations and Feynman diagrams, J. High Energy Phys. 09 (2015) 136.

[33] C. Cardona, B. Feng, H. Gomez, and R. Huang, Cross-ratio identities and higher-order poles of CHY-integrand, J. High Energy Phys. 09 (2016) 133.

[34] T. Adamo, E. Casali, and D. Skinner, Ambitwistor strings and the scattering equations at one loop, J. High Energy Phys. 04 (2014) 104.

[35] T. Adamo and E. Casali, Scattering equations, supergravity integrands, and pure spinors, J. High Energy Phys. 05 (2015) 120.

[36] Y. Geyer, L. Mason, R. Monteiro, and P. Tourkine, Loop Integrands for Scattering Amplitudes from the Riemann Sphere, Phys. Rev. Lett. 115, 121603 (2015).

[37] Y. Geyer, L. Mason, R. Monteiro, and P. Tourkine, One-loop amplitudes on the Riemann sphere, J. High Energy Phys. 03 (2016) 114.

[38] C. Baadsgaard, N. E. J. Bjerrum-Bohr, J. L. Bourjaily, P. H. Damgaard, and B. Feng, Integration rules for loop scattering equations, J. High Energy Phys. 11 (2015) 080.

[39] C. Cardona and H. Gomez, CHY-graphs on a torus, J. High Energy Phys. 10 (2016) 116.

[40] S. He and O. Schlotterer, New Relations for Gauge-Theory and Gravity Amplitudes at Loop Level, Phys. Rev. Lett. 118, 161601 (2017).

[41] H. Gomez, S. Mizera, and G. Zhang, CHY loop integrands from holomorphic forms, J. High Energy Phys. 03 (2017) 092.

[42] H. Gomez, Quadratic Feynman loop integrands from massless scattering equations, Phys. Rev. D 95, 106006 (2017).

[43] H. Gomez, C. Lopez-Arcos, and P. Talavera, One-loop Parke-Taylor factors for quadratic propagators from massless scattering equations, J. High Energy Phys. 10 (2017) 175 . 
[44] Y. Geyer and R. Monteiro, Gluons and gravitons at one loop from ambitwistor strings, J. High Energy Phys. 03 (2018) 068.

[45] N. Ahmadiniaz, H. Gomez, and C. Lopez-Arcos, Non-planar one-loop Parke-Taylor factors in the CHY approach for quadratic propagators, J. High Energy Phys. 05 (2018) 055.

[46] J. Agerskov, N.E. J. Bjerrum-Bohr, H. Gomez, and C. Lopez-Arcos, One-loop Yang-Mills integrands from scattering equations, Phys. Rev. D 102, 045023 (2020).

[47] B. Feng, CHY-construction of planar loop integrands of cubic scalar theory, J. High Energy Phys. 05 (2016) 061.

[48] Y. Geyer, L. Mason, R. Monteiro, and P. Tourkine, Twoloop scattering amplitudes from the Riemann sphere, Phys. Rev. D 94, 125029 (2016).

[49] J. A. Farrow, Y. Geyer, A. E. Lipstein, R. Monteiro, and R. Stark-Muchão, Propagators, BCFW recursion and new scattering equations at one loop, J. High Energy Phys. 10 (2020) 074.
[50] Y. Geyer, R. Monteiro, and R. Stark-Muchão, Two-loop scattering amplitudes: Double-forward limit and colourkinematics duality, J. High Energy Phys. 12 (2019) 049.

[51] Y. Geyer and R. Monteiro, Two-loop scattering amplitudes from ambitwistor strings: From genus two to the nodal Riemann sphere, J. High Energy Phys. 11 (2018) 008.

[52] F. Cachazo, S. He, and E. Y. Yuan, One-loop corrections from higher dimensional tree amplitudes, J. High Energy Phys. 08 (2016) 008.

[53] S. He and E. Y. Yuan, One-loop scattering equations and amplitudes from forward limit, Phys. Rev. D 92, 105004 (2015).

[54] B. Feng and C. Hu, One-loop CHY-integrand of bi-adjoint scalar theory, J. High Energy Phys. 02 (2020) 187.

[55] B. Feng and Y. Zhang, Note on the labelled tree graphs, J. High Energy Phys. 12 (2020) 096.

[56] F. Cachazo and A. Strominger, Evidence for a new soft graviton theorem, arXiv:1404.4091. 